\title{
GW invariants and invariant quotients
}

\author{
Mihai Halic
}

\begin{abstract}
Given a complex, projective variety and a connected, reductive group acting on it, we investigate the relationship between the Gromov-Witten invariants of the variety and those of its invariant quotient for the group action. Certain so-called Hamiltonian invariants naturally appear in the context.
\end{abstract}

Mathematics Subject Classification (2000). 14H10, 14L30, 14N35.

Keywords. Gromov-Witten invariants, group actions, Hamiltonian invariants.

\section{Introduction}

Gromov-Witten theory became a major tool in enumerative geometry because the Gromov-Witten invariants (GW-invariants for short) give in some cases the number of curves in projective varieties, satisfying certain incidence conditions. However, it is usually quite difficult to explicitly compute these invariants, and therefore it is useful to know their behavior under 'modifications' of the variety we start with; typical examples are the blow-up or the symplectic connected sum.

In the present paper we are dealing with group actions. The starting point of this study was the question: given a projective variety and a group acting on it, is there any relationship between the GW-invariants of this variety and those of its quotient for the group action? In this form, it is rather hopeless to answer the question, so that it eventually became: given a projective variety $X$ with a very ample line bundle $\mathcal{O}_{X}(1) \rightarrow X$ and a connected, linearly reductive group $G$ whose action on $X$ is linearized in $\mathcal{O}_{X}(1)$, is there any relationship between the $\mathrm{GW}$-invariants of $X$ and those of its invariant quotient $X / / G$, under the additional assumption that $G$ acts freely on the $G$-semi-stable locus of $X$ ? Certainly, such an assumption makes the geometric invariant theory on $X$ trivial because in this case $X^{\text {ss }} \rightarrow X / / G$ is simply a principal $G$-bundle. We shall see however, that this assumption appears quite naturally in the context, and even so we will have to face rather complicated situations.

The strategy adopted to attack the question above is the following: the $G$ - 
action on $X$ induces one on the space of stable maps to $X$ and two maps in the same $G$-orbit, with image contained in the semi-stable locus of $X$, induce the same map into the quotient. The first idea which comes to mind is to compare the invariant quotient, for this action, of the moduli space of stable maps to $X$ with the moduli space of stable maps to the quotient $X / / G$. For a reason which will become clear in a moment, this strategy is correct only in genus zero, for in higher genera changes are needed.

Let me present more precisely the setting of the problem: the group $G$ and the variety $X$ are as above, and $A \in H_{2}(X ; \mathbb{Z})$ is a homology class which can be represented by a morphism $C \rightarrow X^{\mathrm{ss}}$, with $C$ a smooth projective curve of genus $g$; we let $\hat{A}:=\phi_{*} A \in H_{2}(X / / G ; \mathbb{Z})$ be the push-forward for the projection $\phi: X^{\mathrm{ss}} \rightarrow X / / G$. What we would like to compute are the $\mathrm{GW}$-invariants of $X / / G$ corresponding to the class $\hat{A}$.

For making clear the logic of the article, I shall start with a naive comparison of the expected dimensions of the moduli spaces of stable maps involved here (the precise meaning of the notations will be given later on):

$$
\begin{aligned}
\hat{D} & :=\exp \cdot \operatorname{dim} \bar{M}_{g, k}(X / / G, \hat{A})=(3-\operatorname{dim} X / / G)(g-1)+c_{1}(X / / G) \cdot \hat{A}+k, \\
D-\operatorname{dim} G & :=\exp \cdot \operatorname{dim} \cdot \bar{M}_{g, k}(X, A) / / G \\
& =(3-\operatorname{dim} X)(g-1)+c_{1}(X) \cdot A+k-\operatorname{dim} G,
\end{aligned}
$$

and therefore

$$
\hat{D}-(D-\operatorname{dim} G)=g \cdot \operatorname{dim} G .
$$

From this computation we deduce that in general the space $\bar{M}_{g, k}(X / / G, \hat{A})$ is larger than $\bar{M}_{g, k}(X, A) / / G$, the only exception happening in genus zero. Very shortly, the explanation for this phenomenon is that the projective line is the only one smooth curve which has the property that the trivial principal $G$-bundle over it is rigid. In higher genera, the trivial principal $G$-bundle can be deformed in 'several directions', whose number agrees with the difference $(\star)$ above. This is the reason why for computing higher genus invariants of $\hat{X}$ we will need to consider maps into a larger variety $\bar{X}$ whose construction, in the case when $G$ is a torus, is given in lemma 6.1 .

The article is organized as follows: the first section recalls some basic facts about stable maps and their moduli spaces, the reference being [9].

In section 2 we describe the $G$-semi-stable points of the moduli space of stable maps $\bar{M}_{g, k}(X, A)$. The results obtained in this section hold in full generality, no matter what the $G$-action on $X$ looks like. We obtain the sufficient result (theorem $2.5)$ which says that a map with image contained in the semi-stable locus of $X$ is $G$-semi-stable as a point of $\bar{M}_{g, k}(X, A)$ and a necessary result (corollary 2.4) which says that a stable map representing a $G$-semi-stable point of $\bar{M}_{g, k}(X, A)$ does not have its image contained in the unstable locus of $X$.

Section 3 characterizes the semi-stable points of $\bar{M}_{g, k}(X, A)$ from a symplectic point of view, which will be useful later on in section 6 where we will give an algebro-geometric construction of the space of maps needed for defining certain 
'Hamiltonian invariants'. We compute an explicit formula for the moment map on the space of stable maps which corresponds to a $\mathbb{C}^{*}$-action (proposition 3.5 ), and using it we give (theorem 3.7) a second proof for theorem 2.5.

Finally, section 4 closes the first part of the article giving a partial answer to the initial problem, that of comparing the genus zero GW-invariants of $X$ and $X / / G$. Theorem 4.2 states, under certain transversality assumptions which are technical in nature, that if $G$ acts freely on the semi-stable locus of $X, A$ is a spherical class for which a representative may be found to lie entirely in the stable locus $X^{\text {ss }}$ and $\hat{A}$ denotes the push-forward class in $X / / G$, then

$$
G W_{X / / G, \hat{A}}^{0, k}\left(\hat{a}_{1}, \ldots, \hat{a}_{k}\right)=G W_{X, A}^{0, k}\left(a_{1} \zeta, \ldots, a_{k}\right) .
$$

In this equality the $a_{i}$ 's are the classes on $X$ determined by the $\hat{a}_{i}$ 's on $X / / G$ via the rational quotient map (essentially by pull-back), and $\zeta$ denotes the class of a rational slice for $X \rightarrow X / / G$. We conclude the section with some explicit computations.

Sections 5, 6 and 7 grew out from the attempt at understanding from an algebraic point of view the Hamiltonian invariants defined in [6] and [14], in the case of torus actions. Maybe I should say a word about the origin of this interest: on one hand, in sections 2 and 3 we have tried to develop the algebraic tools needed for computing the GW-invariants of a quotient variety while, on the other hand, the papers [6] and [14] bring into the scene some new invariants, constructed using real-analytic methods, which are associated to Hamiltonian group actions on symplectic manifolds. For particular choices of certain parameters, these ones should compute the GW-invariants of the Marsden-Weinstein quotient of the symplectic variety on which the group acts. Since in the case of projective varieties the symplectic reduction and the geometric invariant quotient basically agree, it was natural to try relating the two constructions: the algebro-geometric and the real-analytic one.

It turns out that the construction of these 'Hamiltonian invariants' fits well in the frame of the previous sections. We identify (proposition 6.2 ) the moduli spaces introduced in $[6,14]$ with the space of stable maps into the variety $\bar{X}:=\mathcal{P} \times\left(\mathbb{C}^{*}\right)^{r} X$, where $\mathcal{P} \rightarrow\left(\mathrm{Pic}^{0} \mathrm{C}\right)^{r} \times C$ denotes a Poincaré bundle parameterizing principal $G=\left(\mathbb{C}^{*}\right)^{r}$-bundles over the curve $C$, so that the real-analytic and the algebraic points of view actually coincide.

In the last section we prove (theorem 7.2), under the same transversality assumptions as in theorem 4.2 , the conjecture formulated in [6] which states that for certain choices of the parameters these Hamiltonian invariants of $X$ coincide with Gromov-Witten invariants on the quotient $X / / G$.

Acknowledgements. I thank both referees for their remarks which have helped me to improve the presentation of this article and also for pointing out errors in the original manuscript. 


\section{Some preparatory material}

In this section I shall recall from [9] the definition of a stable map, the construction of the space $\bar{M}_{g, k}(X, A)$ as a projective scheme and the description of an ample line bundle on it.

In the whole paper $X$ denotes a complex projective, irreducible variety and $\mathcal{O}_{X}(1) \rightarrow X$ denotes a very ample line bundle on it; let $X \hookrightarrow \mathbb{P}^{r}$ be the embedding given by the linear system of $\mathcal{O}_{X}(1)$.

Definition 1.1. A stable map $[(C, \underline{x}, u)]$ to $X$ consists of the following data:

(i) a connected, reduced, complete algebraic curve $(C, \underline{x})$ with $k$ distinct marked points $\underline{x}=\left(x_{1}, \ldots, x_{k}\right)$. The singularities of $C$ are at most ordinary double points and the markings lie in the smooth locus of $C$;

(ii) the equivalence class of a morphism $u:(C, \underline{x}) \rightarrow X$. Two morphism $u:(C, \underline{x}) \rightarrow X$ and $u^{\prime}:\left(C^{\prime}, \underline{x}^{\prime}\right) \rightarrow X$ are equivalent if there exists an isomorphism $\gamma: C \rightarrow C^{\prime}$ such that $\gamma\left(x_{j}\right)=x_{j}^{\prime}$ for $j=1, \ldots, k$ and $u=u^{\prime} \circ \gamma$;

(iii) the stability means that the automorphism group of the map $(C, \underline{x}, u)$ is finite.

By abuse of language, we shall often call a stable map and denote it by $(C, \underline{x}, u)$, the morphism $u:(C, \underline{x}) \rightarrow X$ itself (satisfying (i) $+($ iii)) and not the equivalence class defined by it.

A stable map $[(C, \underline{x}, u)]$ is said to represent the 2-homology class $A \in H_{2}(X ; \mathbb{Z})$ if $u_{*}[C]=A$. Composing such a map with the inclusion $X \hookrightarrow \mathbb{P}^{r}$, one obtains a stable map to $\mathbb{P}^{r}$ which represents an integral multiple $d$ of the class $\ell$ of a line in $\mathbb{P}^{r}$. In the sequel we shall briefly recall the construction of the moduli space $\bar{M}_{g, k}\left(\mathbb{P}^{r}, d\right)$ of stable maps of genus $g$, with $k$ marked points, representing the class $d \ell \in H_{2}\left(\mathbb{P}^{r} ; \mathbb{Z}\right)$. The moduli space $\bar{M}_{g, k}(X, A)$ will be a closed subscheme of it.

It is immediate that the stability condition is equivalent to the fact that the line bundle $L_{(C, x, u)}:=\omega_{C}\left(x_{1}+\cdots+x_{k}\right) \otimes u^{*} \mathcal{O}_{X}(3) \rightarrow C$ is ample, and a combinatorial argument proves that there is an integer $f \geq 1$ having the property that for any stable map $(C, \underline{x}, u)$ to $\mathbb{P}^{r}$ of genus $g$ and with $k$ marked points, representing the class $d \ell \in H_{2}\left(\mathbb{P}^{r} ; \mathbb{Z}\right)$, the line bundle $L_{(C, \underline{x}, u)}^{\otimes f} \rightarrow C$ is very ample and $H^{1}\left(C, L_{(C, \underline{x}, u)}^{\otimes f}\right)=0$. Fix such an integer $f$ once for all. For any $k$-pointed, genus $g$ stable map $(C, \underline{x}, u)$ representing the class $d \ell$ one gets an embedding

$$
u_{Y}:=\left(\left|L_{(C, \underline{x}, u)}^{\otimes f}\right|, u\right): C \longrightarrow \mathbb{P}\left(H^{0}\left(C, L_{(C, \underline{x}, u)}^{\otimes f}\right)^{\vee}\right) \times \mathbb{P}^{r} \cong \mathbb{P}^{N} \times \mathbb{P}^{r}=: Y,
$$

into a product of two projective spaces. The dimension of the projective spaces $\mathbb{P}\left(H^{0}\left(C, L_{(C, \underline{x}, u)}^{\otimes f}\right)^{\vee}\right)$ is independent of the choice of the stable map with the properties mentioned above and is given by a Riemann-Roch formula. Notice that $C$ is determined as a subvariety of $Y$ up to a $P G l(N+1)$-action and that the Hilbert polynomial $P$ of $C$ inside $Y$ does not depend on the choice of the stable map $(C, \underline{x}, u)$. Denoting by $\mathcal{H}:=\mathcal{H} i b_{Y}^{P}$ the Hilbert scheme of closed subschemes of $Y$ 
whose Hilbert polynomial is $P$, to each stable map $(C, \underline{x}, u)$ one associates a point in $\mathcal{H} \times Y^{k}$ as follows:

$$
(C, \underline{x}, u) \mapsto\left(u_{Y *} C, u_{Y}\left(x_{1}\right), \ldots, u_{Y}\left(x_{k}\right)\right) .
$$

The natural $P G l(N+1)$-action on $\mathbb{P}^{N}$ induces an action on $\mathcal{H} \times Y^{k}$ and two stable maps $\left(C_{1,2}, \underline{x}_{1,2}, u_{1,2}\right)$ are isomorphic if and only if they are in the same $P G l(N+1)$-orbit. The stability condition translates into the fact that the stabilizer of any stable map under this action is finite.

It is proved in [9] that there is a certain subscheme $\mathcal{S}$ of $\mathcal{H} \times Y^{k}$ such that $\bar{M}_{g, k}\left(\mathbb{P}^{r}, d\right)=\mathcal{S} / P G l(N+1)$. One of the main results obtained in that paper is that $\bar{M}_{g, k}\left(\mathbb{P}^{r}, d\right)$ is a separated and proper scheme, projective over $\mathbb{C}$. Following [9], we are going to describe an ample line bundle on $\bar{M}_{g, k}\left(\mathbb{P}^{r}, d\right)$. The Hilbert scheme $\mathcal{H}$ is projective, an embedding of it into a projective space being obtained as follows: $\mathcal{L}:=\mathcal{O}_{\mathbb{P}^{N}}(1) \otimes \mathcal{O}_{\mathbb{P}^{r}}(1) \rightarrow Y$ is a very ample line bundle and for large enough integral values of $l$, the restriction homomorphism

$$
\mathcal{W}:=H^{0}\left(Y, \mathcal{L}^{l}\right)^{(0)} \oplus \bigoplus_{j=1}^{k} H^{0}\left(Y, \mathcal{L}^{l}\right)^{(j)} \longrightarrow H^{0}\left(Y, \mathcal{O}_{C} \otimes \mathcal{L}^{l}\right) \oplus \bigoplus_{j=1}^{k} \mathcal{L}_{x_{j}}^{l}
$$

is surjective at each point $\left(C, x_{1}, \ldots, x_{k}\right) \in \mathcal{H} \times Y^{k}$. For distinguishing between the different direct summands of $\mathcal{W}$, we have used upper indices for the same vector space $H^{0}\left(Y, \mathcal{L}^{l}\right)$. Moreover, under the same assumption that $l$ is large enough, the dimension

$$
\operatorname{dim} H^{0}\left(Y, \mathcal{O}_{C} \otimes \mathcal{L}^{l}\right) \oplus \bigoplus_{j=1}^{k} \mathcal{L}_{x_{j}}^{l}=P(l)+k=: q+k
$$

is independent of the point $\left(C, x_{1}, \ldots, x_{k}\right) \in \mathcal{H} \times Y^{k}$.

It is proved in [7] that the map

$$
\begin{aligned}
\mathcal{H} \times Y^{k} & \longrightarrow G r_{q+k}\left(\mathcal{W}^{\vee}\right) \\
(C, \underline{x}) & \mapsto\left(H^{0}\left(Y, \mathcal{O}_{C} \otimes \mathcal{L}^{l}\right) \oplus \bigoplus_{j=1}^{k} \mathcal{L}_{x_{j}}^{l}\right)^{\vee}
\end{aligned}
$$

is a closed immersion. Composing it with the usual projective embedding of the Grassmann variety, one obtains the projective embedding of $\mathcal{H} \times Y^{k}$ :

$$
\begin{aligned}
& \mathcal{H} \times Y^{k} \longrightarrow \mathbb{P}\left(\bigwedge^{q+k} \mathcal{W}^{\vee}\right) \\
& (C, \underline{x}) \mapsto \operatorname{det}\left(\left(H^{0}\left(Y, \mathcal{O}_{C} \otimes \mathcal{L}^{l}\right) \oplus \bigoplus_{j=1}^{k} \mathcal{L}_{x_{j}}^{l}\right)\right)
\end{aligned}
$$


The induced ample line bundle on $\mathcal{H} \times Y^{k}$ is

$$
\operatorname{det} \mathcal{Q}_{k}:=\operatorname{det}\left(\mathcal{Q} \boxplus\left(\mathcal{L}^{l}\right)^{\boxplus k}\right)
$$

where $\mathcal{Q} \rightarrow G r_{q}\left(H^{0}\left(Y, \mathcal{L}^{l}\right)^{\vee}\right)$ is the universal quotient bundle. The fibre of $\mathcal{Q}_{k}$ at a point $(C, \underline{x}) \in \mathcal{H} \times Y^{k}$ is $\mathcal{Q}_{k(C, \underline{x})}=H^{0}\left(Y, \mathcal{O}_{C} \otimes \mathcal{L}^{l}\right) \oplus \bigoplus_{j=1}^{l} \mathcal{L}_{x_{j}}^{l}$. Since $\mathcal{Q}_{k}$ is invariant under the $\operatorname{PGl}(N+1)$-action, $\operatorname{det} \mathcal{Q}_{k}$ is also. It is proved in [9] that $\operatorname{det} \mathcal{Q}_{k}$ descends to an ample line bundle on $\bar{M}_{g, k}(X, A)=\mathcal{S} / P G l(N+1)$.

\section{The semi-stable points on $\bar{M}_{g, k}(X, A)$}

Here is the setup: $G$ is a connected, linearly reductive, complex algebraic group which acts on the complex, irreducible, projective variety $X$, and we choose a very ample line bundle $\mathcal{O}_{X}(1) \rightarrow X$ which is $G$-linearized. This action naturally induces one on the space of stable maps by

$$
\begin{gathered}
G \times \bar{M}_{g, k}(X, A) \longrightarrow \bar{M}_{g, k}(X, A), \\
(g,[(C, \underline{x}, u)]) \mapsto[(C, \underline{x}, g u)],
\end{gathered}
$$

where $g u: C \rightarrow X$ is defined by $(g u)(p):=g \cdot u(p)$ for all $p \in C$. In order to compute the geometric invariant quotient of $\bar{M}_{g, k}(X, A)$, we need a linearization of the action in an ample line bundle.

The linearized $G$-action on $X$ extends to a linearized action on

$$
\mathcal{O}_{\mathbb{P}^{r}}(1) \rightarrow \mathbb{P}^{r}:=\mathbb{P}\left(H^{0}\left(X, \mathcal{O}_{X}(1)\right)^{\vee}\right)
$$

such that $X$ is invariant. We have already mentioned in the previous section that $\bar{M}_{g, k}(X, A)$ is a closed subscheme of $\bar{M}_{g, k}\left(\mathbb{P}^{r}, d\right)$ and on this last one we have described an ample line bundle. Our next task is to linearize the $G$-action in it.

Using the notations of the previous section, $\bar{M}_{g, k}\left(\mathbb{P}^{r}, d\right)=\mathcal{S} / P G l(N+1)$, where $\mathcal{S}$ is some subscheme of $\mathcal{H} \times Y^{k}$. On $\mathcal{H} \times Y^{k}$ there are two actions: the first one is the $P G l(N+1)$-action on $\mathcal{H}$ and the second one is the $G$-action induced by that on the factor $\mathbb{P}^{r}$ in $Y=\mathbb{P}^{N} \times \mathbb{P}^{r}$. Since the $P G l(N+1)$ and the $G$-actions on $Y$ commute, the induced actions on $\mathcal{H} \times Y^{k}$ commute also. For this reason, $G$-semi-stable points on $\bar{M}_{g, k}\left(\mathbb{P}^{r}, d\right)$ will be the images of $G$-semi-stable points of $\mathcal{S}$; therefore it is enough to describe the linearized $G$-action on $\operatorname{det} \mathcal{Q}_{k} \rightarrow \mathcal{H} \times Y^{k}$. Since $G$ acts on $\mathcal{L} \rightarrow Y$, it acts also on $H^{0}\left(Y, \mathcal{L}^{l}\right)$ by $(g S)_{y}:=g S_{g^{-1} y}$ for all $S \in H^{0}\left(Y, \mathcal{L}^{l}\right)$ and all $y \in Y$. The dual action on $H^{0}\left(Y, \mathcal{L}^{l}\right)^{\vee}$ is given by $(g, \Sigma) \mapsto g \Sigma$, where $\langle g \Sigma, S\rangle:=\left\langle\Sigma, g^{-1} S\right\rangle$. The induced action on $\bigwedge^{q+k} \mathcal{W}^{\vee}$ is now obvious because $\mathcal{W}=H^{0}\left(Y, \mathcal{L}^{l}\right)^{(0)} \oplus \bigoplus_{j=1}^{k} H^{0}\left(Y, \mathcal{L}^{l}\right)^{(j)}$.

For finding the $G$-semi-stable points we shall use the Hilbert-Mumford criterion. Given $\lambda: \mathbb{C}^{*} \rightarrow G$ a one parameter subgroup of $G$ (a 1-PS for short), there 
is a (finite) direct sum decomposition corresponding to the characters of $\mathbb{C}^{*}$ :

$$
\begin{gathered}
H^{0}\left(Y, \mathcal{L}^{l}\right)=\bigoplus_{m \in \mathbb{Z}} H^{0}\left(Y, \mathcal{L}^{l}\right)_{m}, \\
\lambda(t) S=t^{m} S \quad \forall t \in \mathbb{C}^{*} \quad \forall S \in H^{0}\left(Y, \mathcal{L}^{l}\right)_{m} .
\end{gathered}
$$

We want to find out when the point

$$
\left[\bigwedge^{q+k}\left(H^{0}\left(Y, \mathcal{O}_{C} \otimes \mathcal{L}^{l}\right)^{(0)} \oplus \bigoplus_{j=1}^{k} \mathcal{L}_{x_{j}}^{l}\right)^{\vee}\right] \in \mathbb{P}\left(\bigwedge^{q+k} \mathcal{W}^{\vee}\right)
$$

is $\lambda$-semi-stable, so we have to study the $\mathbb{C}^{*}$-orbit of a representative of this point $\operatorname{in} \bigwedge^{q+k} \mathcal{W}^{\vee}$.

Let $\sigma_{1}, \ldots, \sigma_{q}$ be a basis of $\operatorname{Hom}_{\mathbb{C}}\left(H^{0}\left(C, \mathcal{L}^{l}\right)^{(0)}, \mathbb{C}\right)$ and $\tau_{1}, \ldots, \tau_{k}$ be generators of $\operatorname{Hom}\left(\mathcal{L}_{x_{j}}^{l}, \mathbb{C}\right), j=1, \ldots, k$. Notice that the choice of the $\tau_{j}$ 's is equivalent to the choice of representatives $x_{j}^{\prime} \in \mathbb{C}^{N} \times \mathbb{C}^{r+1}$ of $x_{j}=\left(x_{j, 1}, x_{j, 2}\right) \in Y$ because $\mathcal{L}_{x_{j}}=\mathcal{O}_{\mathbb{P}^{N-1}}(1)_{x_{j, 1}} \otimes \mathcal{O}_{\mathbb{P}^{r}}(1)_{x_{j, 2}}$. Using the epimorphism

$$
\mathcal{W}=H^{0}\left(Y, \mathcal{L}^{l}\right)^{(0)} \oplus \bigoplus_{j=1}^{k} H^{0}\left(Y, \mathcal{L}^{l}\right)^{(j)} \stackrel{\imath_{C}}{\longrightarrow} H^{0}\left(C, \mathcal{L}^{l}\right) \oplus \bigoplus_{j=1}^{k} \mathcal{L}_{x_{j}}^{l} \longrightarrow 0
$$

$\sigma_{1}, \ldots, \sigma_{q}, \tau_{1}, \ldots, \tau_{k}$ can be extended to linear functionals on $\mathcal{W}$

$$
\begin{gathered}
\mathcal{S}_{1}, \ldots, \mathcal{S}_{q}: H^{0}\left(Y, \mathcal{L}^{l}\right)^{(0)} \longrightarrow \mathbb{C} \\
\left\langle\mathcal{S}_{j}, S\right\rangle:=\left\langle\sigma_{j}, \imath_{C} S\right\rangle \quad j=1, \ldots, q \quad \forall S \in H^{0}\left(Y, \mathcal{L}^{l}\right)^{(0)}
\end{gathered}
$$

and

$$
\begin{gathered}
\mathcal{T}_{1}, \ldots, \mathcal{T}_{k}: H^{0}\left(Y, \mathcal{L}^{l}\right) \longrightarrow \mathbb{C} \\
\left\langle\mathcal{T}_{j}, S\right\rangle:=\left\langle\tau_{j}, S\left(x_{j}\right)\right\rangle \quad j=1, \ldots, k \quad \forall S \in H^{0}\left(Y, \mathcal{L}^{l}\right)^{(j)} .
\end{gathered}
$$

The $\mathcal{T}_{j}$ 's represent just the evaluations of the homogeneous polynomial $S$ at the points $x_{j}^{\prime}$ representing $x_{j}$.

Let us remark that the linear functionals $\mathcal{S}_{j}$ act only on $H^{0}\left(Y, \mathcal{L}^{l}\right)^{(0)}$ and evaluate identically to zero on the other copies $H^{0}\left(Y, \mathcal{L}^{l}\right)^{(j)}, j \neq 0$. A similar remark is valid for the $\mathcal{T}_{j}$ 's: they evaluate identically to zero on $H^{0}\left(Y, \mathcal{L}^{l}\right)^{\left(j^{\prime}\right)}, j^{\prime} \neq j$.

The semi-stability condition reads

$$
0 \notin \overline{\mathbb{C}^{*} \cdot \mathcal{S}_{1} \wedge \cdots \wedge \mathcal{S}_{q} \wedge \mathcal{T}_{1} \wedge \cdots \wedge \mathcal{T}_{k}} \wedge^{q+k} \mathcal{W} \vee
$$

which is equivalent to the existence of $S_{1}, \ldots, S_{q+k}, S_{1}^{\prime}, \ldots, S_{q+k}^{\prime} \in \mathcal{W}$ such that

$$
\left\{\begin{array}{l}
0 \neq \lim _{t \rightarrow 0}\left\langle\lambda(t) \cdot\left(\mathcal{S}_{1} \wedge \cdots \wedge \mathcal{S}_{q} \wedge \mathcal{T}_{1} \wedge \cdots \wedge \mathcal{T}_{k}\right), S_{1} \wedge \cdots \wedge S_{q+k}\right\rangle, \\
0 \neq \lim _{t \rightarrow \infty}\left\langle\lambda(t) \cdot\left(\mathcal{S}_{1} \wedge \cdots \wedge \mathcal{S}_{q} \wedge \mathcal{T}_{1} \wedge \cdots \wedge \mathcal{T}_{k}\right), S_{1}^{\prime} \wedge \cdots \wedge S_{q+k}^{\prime}\right\rangle .
\end{array}\right.
$$


Each vector $S_{j}, S_{j}^{\prime}$ is the sum of $1+k$ vectors corresponding to the direct sum decomposition of $\mathcal{W}$. Moreover, as pointed out before, each of the $\mathcal{S}_{j}$ 's and $\mathcal{T}_{j}$ 's evaluate non-zero only on vectors in a certain component of $\mathcal{W}$. Consequently, for this last condition to be satisfied, one may assume that

$$
S_{1}, S_{1}^{\prime} \ldots, S_{q}, S_{q}^{\prime} \in H^{0}\left(Y, \mathcal{L}^{l}\right)^{(0)} \quad \text { and } \quad S_{q+j}, S_{q+j}^{\prime} \in H^{0}\left(Y, \mathcal{L}^{l}\right)^{(j)} j=1, \ldots, k .
$$

Since the $\mathbb{C}^{*}$-action of the 1-PS $\lambda$ of $G$ induces the decomposition $H^{0}\left(Y, \mathcal{L}^{l}\right)$ $=\oplus_{m \in \mathbb{Z}} H^{0}\left(Y, \mathcal{L}^{l}\right)_{m}$, we can further assume that

$$
S_{j} \in H^{0}\left(Y, \mathcal{L}^{l}\right)_{m_{j}}^{(0)}, \quad S_{j}^{\prime} \in H^{0}\left(Y, \mathcal{L}^{l}\right)_{m_{j}^{\prime}}^{(0)} \quad \text { for } \quad j=1, \ldots, q
$$

and

$$
S_{q+j} \in H^{0}\left(Y, \mathcal{L}^{l}\right)_{m_{q+j}}^{(j)}, \quad S_{q+j}^{\prime} \in H^{0}\left(Y, \mathcal{L}^{l}\right)_{m_{q+j}^{\prime}}^{(j)} \quad \text { for } \quad j=1, \ldots, k .
$$

We are now going to compute the first condition in (2.1):

$$
\begin{aligned}
& \left\langle\lambda(t) \cdot\left(\mathcal{S}_{1} \wedge \cdots \wedge \mathcal{S}_{q} \wedge \mathcal{T}_{1} \wedge \cdots \wedge \mathcal{T}_{k}\right), S_{1} \wedge \ldots S_{q} \wedge S_{q+1} \wedge \cdots \wedge S_{q+k}\right\rangle \\
& =\left\langle\mathcal{S}_{1} \wedge \cdots \wedge \mathcal{S}_{q} \wedge \mathcal{T}_{1} \wedge \cdots \wedge \mathcal{T}_{k},\right. \\
& \left.\quad \lambda\left(t^{-1}\right) S_{1} \wedge \cdots \wedge \lambda\left(t^{-1}\right) S_{q} \wedge \lambda\left(t^{-1}\right) S_{q+1} \wedge \cdots \wedge \lambda\left(t^{-1}\right) S_{q+k}\right\rangle \\
& =\left\langle\mathcal{S}_{1} \wedge \cdots \wedge \mathcal{S}_{q} \wedge \mathcal{T}_{1} \wedge \cdots \wedge \mathcal{T}_{k},\right. \\
& \left.\quad t^{-m_{1}} S_{1} \wedge \cdots \wedge t^{-m_{q}} S_{q} \wedge t^{-m_{q+1}} S_{q+1} \wedge \cdots \wedge t^{-m_{q+k}} S_{q+k}\right\rangle \\
& =t^{-\sum_{j=1}^{q+k} m_{j}} \cdot\left\langle\mathcal{S}_{1} \wedge \cdots \wedge \mathcal{S}_{q}, S_{1} \wedge \cdots \wedge S_{q}\right\rangle \cdot \prod_{j=1}^{k}\left\langle\mathcal{T}_{j}, S_{q+j}\right\rangle \\
& =t^{-\sum_{j=1}^{q+k} m_{j}} \cdot\left\langle\sigma_{1} \wedge \cdots \wedge \sigma_{q}, \imath_{C}^{*} S_{1} \wedge \cdots \wedge \imath_{C}^{*} S_{q}\right\rangle \cdot \prod_{j=1}^{k} S_{q+j}\left(x_{j}^{\prime}\right) .
\end{aligned}
$$

Doing the computations corresponding to the second condition in (2.1) we find the

Proposition 2.1. The point $[(C, \underline{x}, u)] \in \bar{M}_{g, k}(X, A)$ is $G$-semi-stable if and only if for any 1 -PS $\lambda: \mathbb{C}^{*} \rightarrow G$ there are sections

$$
S_{1}, S_{1}^{\prime} \ldots, S_{q}, S_{q}^{\prime}, S_{q+1}, S_{q+1}^{\prime}, \ldots, S_{q+k}, S_{q+k}^{\prime} \in H^{0}\left(Y, \mathcal{L}^{l}\right)
$$

satisfying the properties:

$$
\begin{aligned}
& \lambda(t) S_{j}=t^{m_{j}} S_{j} \text { for } j=1, \ldots, q+k \text { with } \sum_{j=1}^{q+k} m_{j} \geq 0 ; \\
& \lambda(t) S_{j}^{\prime}=t^{m_{j}^{\prime}} S_{j} \text { for } j=1, \ldots, q+k \text { with } \sum_{j=1}^{q+k} m_{j}^{\prime} \leq 0 ;
\end{aligned}
$$

(ii) $\left\{\imath_{C}^{*} S_{1}, \ldots, \imath_{C}^{*} S_{q}\right\}$ and $\left\{\imath_{C}^{*} S_{1}, \ldots, \imath_{C}^{*} S_{q}\right\}$ are bases for $H^{0}\left(C, \mathcal{L}^{l}\right)$;

(iii) $S_{q+j}\left(x_{j}\right), S_{q+j}^{\prime}\left(x_{j}\right) \neq 0$ for $j=1, \ldots, k$.

The point $[(C, \underline{x}, u)] \in \bar{M}_{g, k}(X, A)$ is $G$-stable if the same holds, but with strict inequalities in (i). 
The shortcoming of this proposition is being too algebraic and therefore difficult to check in practice. For this reason we shall try to find necessary conditions on one hand and sufficient conditions on the other hand for it. Let us start with the necessary conditions. An easy consequence of the proposition above is the

Corollary 2.2. If the point $[(C, \underline{x}, u)] \in \bar{M}_{g, k}(X, A)$ is G-semi-stable, then for all 1 -PS $\lambda: \mathbb{C}^{*} \rightarrow G$ there are sections

$$
S_{j} \in H^{0}\left(Y, \mathcal{L}^{l}\right)_{m_{j}} \quad j=1, \ldots, q
$$

satisfying the properties:

(i) $\left\{\imath_{C}^{*} S_{1}, \ldots, \imath_{C}^{*} S_{q}\right\}$ is a basis for $H^{0}\left(C, \mathcal{L}^{l}\right)$;

(ii) the set $\left\{m_{j}\right\}_{j=1, \ldots, q}$ contains simultaneously positive and negative integers.

Proof. For a fixed 1-PS of $G$, there are two possibilities in the previous proposition: either all the $m_{j}$ 's vanish for $j=1, \ldots, q$ and we are done, or it is not so. Assume that all $m_{j} \geq 0$ for $j=1, \ldots, q$. Because the sum $\sum_{j=1}^{j=q+k} m_{j}^{\prime} \leq 0$, it follows that there must exist a $m_{q+h}^{\prime} \leq 0$. We know that $S_{q+h}^{\prime}\left(x_{q+h}\right) \neq 0$ and therefore the restriction $\imath_{C}^{*} S_{q+h}^{\prime} \neq 0$. Because $\left\{\imath_{C}^{*} S_{1}, \ldots, \imath_{C}^{*} S_{q}\right\}$ is a basis of $H^{0}\left(C, \mathcal{L}^{l}\right)$, one can write $\imath_{C}^{*} S_{q+h}^{\prime}$ as a non-zero linear combination of these vectors. Now all we have to do is to replace a section from the set $\left\{S_{1}, \ldots, S_{q}\right\}$ which appears in this linear combination with $S_{q+h}^{\prime}$.

The next proposition gives a geometrical restriction which must be satisfied by the $G$-semi-stable maps in $\bar{M}_{g, k}(X, A)$.

Proposition 2.3. If $(C, \underline{x}, u) \in \bar{M}_{g, k}(X, A)$ is a stable map which is G-semistable, then for each 1-PS $\lambda: \mathbb{C}^{*} \rightarrow G$ there is an irreducible component $C_{\delta}$ of $C$ such that the image of the map

$$
u_{\mid C_{\delta}}: C_{\delta} \longrightarrow X
$$

is not contained in the $\lambda$-unstable locus of $X$.

Proof. The line bundle $\mathcal{L}^{l} \rightarrow Y$ is again very ample and its associated linear system gives an embedding

$$
\begin{array}{cc}
\mathcal{L}^{l} & \mathcal{O}_{\mathbb{P}^{R-1}}(1) \\
\downarrow & \downarrow \\
Y & \stackrel{\left|\mathcal{L}^{l}\right|}{\longrightarrow} \\
\mathbb{P}^{R-1}=\mathbb{P}\left(H^{0}\left(Y, \mathcal{L}^{l}\right)^{\vee}\right)
\end{array}
$$

The $G$-action on $\mathcal{L}^{l} \rightarrow Y$ induces one on $\mathcal{O}_{\mathbb{P}^{R-1}}(1) \rightarrow \mathbb{P}^{R-1}$. For a stable map $(C, \underline{x}, u)$ which is $G$-semi-stable and $\lambda: \mathbb{C}^{*} \rightarrow G$ a 1-PS of $G$, corollary 2.2 ensures the existence of sections $S_{j} \in H^{0}\left(Y, \mathcal{L}^{l}\right)_{m_{j}}$ whose restrictions to $C$ give a basis of 
$H^{0}\left(C, \mathcal{L}^{l}\right)$; in particular, they are linearly independent. Because of the direct sum decomposition

$$
H^{0}\left(Y, \mathcal{L}^{l}\right)=\bigoplus_{m \in \mathbb{Z}} H^{0}\left(Y, \mathcal{L}^{l}\right)_{m}
$$

these sections can be completed with sections

$$
S_{q+1} \in H^{0}\left(Y, \mathcal{L}^{l}\right)_{m_{q+1}}, \ldots, S_{R} \in H^{0}\left(Y, \mathcal{L}^{l}\right)_{m_{R}}
$$

to a basis of $H^{0}\left(Y, \mathcal{L}^{l}\right)$. This basis defines coordinates on $H^{0}\left(Y, \mathcal{L}^{l}\right)^{\vee} \cong \mathbb{C}^{R}$ in which the $\lambda$-action is diagonal.

Claim. There exists an irreducible component $C_{\delta}$ of $C$ having the property that among $\left\{S_{1}, \ldots, S_{q}\right\}$ there are two sections $S_{j} \in H^{0}\left(Y, \mathcal{L}^{l}\right)_{m_{j}}$ and $S_{j^{\prime}} \in H^{0}\left(Y, \mathcal{L}^{l}\right)_{m_{j^{\prime}}}$ such that $m_{j^{\prime}} \leq 0$ and $m_{j} \geq 0$ and their restriction to $C_{\delta}$ is non-zero.

We know already that there are two sections $S_{\alpha}$ and $S_{\tau}$ such that $m_{\alpha} \leq 0$ and $m_{\tau} \geq 0$, and their restriction to $C$ is nonzero. Let $C_{\alpha}$ and $C_{\tau}$ respectively two irreducible components of $C$ on which these two sections do not vanish. Because $C$ is connected, there is a chain of irreducible components $C_{\alpha}, C_{\beta}, \ldots, C_{\tau}$ connecting these two components. Since $\left\{S_{1}, \ldots, S_{q}\right\}$ is a basis of $H^{0}\left(C, \mathcal{L}^{l}\right)$ and $\mathcal{L}^{l} \rightarrow C$ is very ample, it follows that there are sections $S_{\alpha \beta}, S_{\beta \gamma}, \ldots, S_{\sigma \tau}$ with the property that: $S_{\alpha \beta}$ does not vanish at a (certain) point in $C_{\alpha} \cap C_{\beta}, S_{\beta \gamma}$ does not vanish at a (certain) point in $C_{\beta} \cap C_{\gamma}, \ldots, S_{\sigma \tau}$ does not vanish at a (certain) point in $C_{\sigma} \cap C_{\tau}$. Notice that $S_{\alpha \beta}$ does not vanish on $C_{\alpha}$ and $C_{\beta}, S_{\beta \gamma}$ does not vanish on $C_{\beta}$ and $C_{\gamma}$ and so on. Let $m_{\alpha \beta}, m_{\beta \gamma}, \ldots$ denote the weights of the sections $S_{\alpha \beta}, S_{\beta \gamma}, \ldots$ respectively. If $m_{\alpha \beta} \geq 0$, then the component $C_{\alpha}$ satisfies the requirement of the claim. If it is not the case, we look at the chain $C_{\beta}, \ldots, C_{\tau}$ whose length is one less than the length of $C_{\alpha}, \ldots, C_{\tau}$. Because at the end $C_{\tau}$ the weight $m_{\tau}$ is positive, an induction argument on the length of the connecting chain shows that it must exist an irreducible component $C_{\delta}$ of the chain $C_{\alpha}, \ldots, C_{\tau}$ having the property of the claim.

Let us look now at the image of a point $p \in C_{\delta}$ inside $\mathbb{P}^{R-1}$ : a representative $p^{\prime} \in \mathbb{C}^{R}$ of it will have non-zero coordinates with both positive and negative weights (for the $\lambda$ action), so $p$ is in the $\lambda$-semi-stable locus of $Y$. Since obviously $Y^{\mathrm{ss}}=\mathbb{P}^{N} \times X^{\mathrm{ss}}$, we deduce that $u(p)$ is in the $\lambda$-semi-stable locus of $X$.

In the case of a torus action, this proposition implies the

Corollary 2.4. Suppose that a torus $T$ acts on $X$. If $(C, \underline{x}, u) \in \bar{M}_{g, k}(X, A)$ is a $T$-semi-stable point and $C$ is irreducible, then the image of $u$ is not contained in the $T$-unstable locus of $X$. 
Proof. By the Hilbert-Mumford criterion,

$$
X_{G}^{\mathrm{ss}}=\bigcap_{\lambda 1-P S \text { of } T} X_{\lambda}^{\mathrm{ss}} .
$$

Since $C$ is assumed irreducible, proposition 2.3 implies that for any 1-PS $\lambda$ of $G$, the image of $u$ intersects the $\lambda$-unstable locus of $X$ in finitely many points; denote by $C^{0}(\lambda)$ the Zariski open subset of $C$ consisting of points which are mapped by $u$ into the $\lambda$-semi-stable locus of $X$. Because in a torus there are countably many one-parameter subgroups,

$$
C \neq \bigcup_{\lambda 1-\mathrm{PS} \text { of } T}\left(C-C^{0}(\lambda)\right)
$$

In what follows we want to prove a weakened converse of proposition 2.3 which is useful when the unstable locus $X^{\text {unstable }}\left(\mathcal{O}_{X}(1)\right)$ has large codimension in $X$. In this case it is reasonable to think that 'many' curves in $X$ won't meet this locus at all.

Theorem 2.5. A stable map $[(C, \underline{x}, u)] \in \bar{M}_{g, k}(X, A)$ having the property that

$$
\text { Image }(u: C \rightarrow X) \subset X^{\mathrm{ss}}\left(\mathcal{O}_{X}(1)\right)
$$

is a $G$-semi-stable point of $\bar{M}_{g, k}(X, A)$.

Proof. The geometric invariant quotient $\widehat{X}=X / / G$ is a projective subvariety of $\widehat{\mathbb{P}^{r}}=\mathbb{P}^{r} / / G$. This last geometric quotient can be described as

$$
\widehat{\mathbb{P}^{r}}=\operatorname{Proj}\left(\sum_{n} H^{0}\left(\mathbb{P}^{r}, \mathcal{O}_{\mathbb{P}^{r}}(n)\right)^{G}\right)
$$

Let's denote by $\phi:\left(\mathbb{P}^{r}\right)^{\mathrm{ss}} \rightarrow \widehat{\mathbb{P}^{r}}$ the quotient map. There is an invertible sheaf $M \rightarrow \widehat{\mathbb{P}^{r}}$ such that $\phi^{*} M=\mathcal{O}_{\mathbb{P}^{r}}\left(m_{0}\right)_{\mid\left(\mathbb{P}^{r}\right)^{\text {ss }}}$ for some $m_{0}>0$ (see [13], Theorem 1.10). It has the additional property that for large enough values of $n$,

$$
H^{0}\left(\widehat{\mathbb{P}^{r}}, M^{n}\right) \stackrel{\phi^{*}}{\cong} H^{0}\left(\mathbb{P}^{r}, \mathcal{O}_{\mathbb{P}^{r}}\left(n m_{0}\right)\right)^{G}
$$

The assumption that the image of the stable map is contained in the semi-stable locus of $X$ implies the existence of the commutative diagram

$$
\begin{gathered}
(C, \underline{x}) \stackrel{u}{\longrightarrow} X^{\mathrm{ss}} \\
\widehat{u} \searrow \swarrow_{\phi} \\
\widehat{X} .
\end{gathered}
$$


Remark 2.6. The map $\widehat{u}:(C, \underline{x}) \rightarrow \widehat{X}$ is still stable. Indeed, problems appear only if $\widehat{u}$ contracts some $\mathbb{P}^{1}$-components, without enough special points on them, which are not contracted by $u$. If $C_{\delta}$ denotes such a $\mathbb{P}^{1}$-component of $C$,

$$
\operatorname{deg}_{u_{*} C_{\delta}} \mathcal{O}_{X}\left(m_{0}\right)=\operatorname{deg}_{\widehat{u}_{*} C_{\delta}} M=0,
$$

so that $u$ must be constant on $C_{\delta}$. This contradicts the stability of $u$.

The group $G$ acts on $Y=\mathbb{P}^{N} \times \mathbb{P}^{r}$ trivially on the first factor and consequently its invariant quotient is $\widehat{Y}:=\mathbb{P}^{N} \times \widehat{\mathbb{P}^{r}}$. The quotient map $\psi: Y^{\mathrm{ss}}(\mathcal{L}) \longrightarrow \widehat{Y}$ is just $\psi=\left(\operatorname{id}_{\mathbb{P}^{N}}, \phi\right)$. Let us define the line bundle

$$
\mathcal{M}:=\mathcal{O}_{\mathbb{P}^{N}}\left(m_{0}\right) \otimes M \longrightarrow \widehat{Y} .
$$

It has the property that

$$
\psi^{*} \mathcal{M}=\mathcal{O}_{\mathbb{P}^{N}}\left(m_{0}\right) \otimes \phi^{*} M=\left(\mathcal{O}_{\mathbb{P}^{N}}\left(m_{0}\right) \otimes \mathcal{O}_{\mathbb{P}^{r}}\left(m_{0}\right)\right)_{\mid Y^{\mathrm{ss}}}=\mathcal{L}^{m_{0}}{ }_{\mid Y^{\mathrm{ss}}}
$$

and it can be easily checked that

$$
H^{0}\left(\widehat{Y}, \mathcal{M}^{n}\right) \stackrel{\psi^{*}}{\cong} H^{0}\left(Y, \mathcal{L}^{n m_{0}}\right)^{G}
$$

for large $n$. There is again a commutative diagram

$$
\begin{gathered}
(C, \underline{x}) \stackrel{u_{Y}}{\longrightarrow} Y^{\mathrm{ss}}=\mathbb{P}^{N} \times\left(\mathbb{P}^{r}\right)^{\mathrm{ss}} \\
\widehat{Y}=\mathbb{P}^{N} \times\left(\operatorname{id}_{\mathbb{P}^{N}}, \phi\right) \\
\widehat{\mathbb{P}^{r}} .
\end{gathered}
$$

Because $u_{Y}$ is an embedding, $\widehat{u}_{Y}$ is also. The 1-dimensional subvariety $\widehat{u}_{Y *} C$ of $\widehat{Y}$ has Hilbert polynomial:

$$
\begin{aligned}
\widehat{P}(n) & =h^{0}\left(C, \widehat{u}_{Y}^{*} \mathcal{M}^{n}\right)-h^{1}\left(C, \widehat{u}_{Y}^{*} \mathcal{M}^{n}\right) \\
& =h^{0}\left(C, u_{Y}^{*} \mathcal{L}^{n m_{0}}\right)-h^{1}\left(C, u_{Y}^{*} \mathcal{L}^{n m_{0}}\right)=P\left(n m_{0}\right),
\end{aligned}
$$

where $P$ is the Hilbert polynomial of $u_{Y *} C \subset Y$. It is independent of $(C, \underline{x}, u) \in$ $\bar{M}_{g, k}(X, A)$ satisfying the hypothesis of the proposition.

Grothendieck proves in [7] that there is an integer $k>0$ such that for all $n \geq k$, $\mathcal{M}^{n}$ is generated by its global sections and moreover, for any closed subscheme $\widehat{Z}$ of $\widehat{Y}$ whose Hilbert polynomial is $\widehat{P}$ there is an epimorphism

$$
H^{0}\left(\widehat{Y}, \mathcal{M}^{n}\right) \longrightarrow H^{0}\left(\widehat{Y}, \mathcal{O}_{\widehat{Z}} \otimes \mathcal{M}\right) \longrightarrow 0 .
$$

Recall that for obtaining a projective embedding of $\bar{M}_{g, k}\left(\mathbb{P}^{r}, d\right)$ we had to chose a high enough power $\mathcal{L}^{l} \rightarrow Y$. Since $\psi^{*} \mathcal{M}=\mathcal{L}^{m_{0}} \mid Y^{\mathrm{ss}}$, we can chose from the very beginning an integer $l$ large enough such that $\psi^{*} \mathcal{M}^{n}=\mathcal{L}^{l}{ }_{\mid Y^{\text {ss }}}$ with $n \geq k$ $\left(l=n m_{0}\right)$. 
The following three relations

$$
\left\{\begin{array}{l}
H^{0}\left(C, u_{Y}^{*} \mathcal{L}^{l}\right)=H^{0}\left(C, \widehat{u}_{Y}^{*} \mathcal{M}^{n}\right) \\
H^{0}\left(\widehat{Y}, \mathcal{M}^{n}\right) \longrightarrow H^{0}\left(C, \widehat{u}_{Y}^{*} \mathcal{M}^{n}\right) \longrightarrow 0 \\
H^{0}\left(\widehat{Y}, \mathcal{M}^{n}\right) \stackrel{\psi^{*}}{\cong} H^{0}\left(Y, \mathcal{L}^{l}\right)^{G}
\end{array}\right.
$$

prove that there are $G$-invariant sections $S_{1}, \ldots, S_{q} \in H^{0}\left(Y, \mathcal{L}^{l}\right)^{G}$ such that the restrictions $\left\{\imath_{C}^{*} S_{1}, \ldots, \imath_{C}^{*} S_{q}\right\}$ form a basis of $H^{0}\left(C, u_{Y}^{*} \mathcal{L}^{l}\right)$. The problem with the marked points is easy: by hypothesis $u\left(x_{1}\right), \ldots, u\left(x_{k}\right) \in X^{\mathrm{ss}}\left(\mathcal{O}_{X}(1)\right)$ and we may consider their images $\widehat{u}_{Y}\left(x_{1}\right), \ldots, \widehat{u}_{Y}\left(x_{k}\right) \in \widehat{Y}$. The number $n$ was chosen large enough to ensure that $\mathcal{M}^{n}$ is globally generated by its sections. Consequently, we find $\widehat{S}_{q+1}, \ldots, \widehat{S}_{q+k} \in H^{0}\left(\hat{Y}, \mathcal{M}^{n}\right)$ such that $\widehat{S}_{q+j}\left(x_{j}\right) \neq 0$ for $j=1, \ldots, k$. Since $H^{0}\left(\widehat{Y}, \mathcal{M}^{n}\right) \stackrel{\psi^{*}}{\cong} H^{0}\left(Y, \mathcal{L}^{l}\right)^{G}$, there are $G$-invariant sections $S_{q+1}, \ldots, S_{q+k} \in$ $H^{0}\left(Y, \mathcal{L}^{l}\right)^{G}$ such that $S_{q+j}\left(x_{j}\right) \neq 0$ for $j=1, \ldots, k$.

The $q+k$ sections $S_{1}, \ldots, S_{q}, S_{q+1}, \ldots, S_{q+k}$ now obviously satisfy the conditions of the proposition 2.1 .

Corollary 2.7. If the stable map $[(C, \underline{x}, u)]$ has the property that Image $u \subset X_{(0)}^{\mathrm{s}}$, then $[(C, \underline{x}, u)] \in \bar{M}_{g, k}(X, A)_{(0)}^{\mathrm{s}}$. As usual, $X_{(0)}^{\mathrm{s}}$ denotes the set of $G$-stable points which have finite stabilizer.

Proof. There are two things to prove in this statement: the first one is that the stabilizer of $[(C, \underline{x}, u)]$ in $G$ is finite, and the second one is that this point is indeed stable.

When $k>0$, for any $x \in \underline{x}$ we have $\operatorname{Stab}_{G}[(C, \underline{x}, u)] \subset \operatorname{Stab}_{G} u(x)$, and therefore the stabilizer of the map is indeed finite. Let us prove that it is so in general. Consider a representative $u:(C, \underline{x}) \rightarrow X$ of the point $[(C, \underline{x}, u)]$ and define $H:=$ $\operatorname{Stab}_{G}[(C, \underline{x}, u)]$. Let's assume that $H$ is not finite. By definition, for any $h \in H$, there is an automorphism $\gamma_{h} \in \operatorname{Aut}(C, \underline{x})$ having the property that $h u=u \gamma_{h}$. In particular, for all $h \in H$, Image $h u=$ Image $u$. For $\zeta \in C$ an arbitrary point, $u(\zeta)$ has finite stabilizer in $G$ by assumption, and therefore $\operatorname{dim} H \cdot u(\zeta)=\operatorname{dim} H>0$. Since $H \cdot u(\zeta) \subset$ Image $u$ which is one dimensional, we deduce that $\operatorname{dim} H=1$. Let us look at the connected component of the identity $H^{\circ}$ of $H$ : it is a connected 1-dimensional group and therefore isomorphic either to the multiplicative group $G_{m}$ or to the additive group $G_{a}$. In both cases $\lim _{t \rightarrow \infty} t \cdot u(\zeta) \in$ Image $u$ will be fixed. This contradicts the assumption that Image $u \stackrel{t \rightarrow \infty}{\subset} X_{(0)}^{\mathrm{s}}$.

We will show that the point $[(C, \underline{x}, u)]$ is $G$-stable using proposition 2.1. For a 1-PS $\lambda: \mathbb{C}^{*} \rightarrow G$, there are sections $S_{1} \in H^{0}\left(Y, \mathcal{L}^{l}\right)_{m_{1}}$ and $S_{1}^{\prime} \in H^{0}\left(Y, \mathcal{L}^{l}\right)_{m_{1}^{\prime}}$ with $m_{1}^{\prime}<0<m_{1}$, and whose restriction to $C$ is non-zero (this is because Image $u \subset$ $\left.X^{\mathrm{ss}}\right)$. Using now $(2.2)$, we complete $S_{1}$ and $S_{1}^{\prime}$ with $G$-invariant sections $S_{j}, S_{j}^{\prime} \in$ $H^{0}\left(Y, \mathcal{L}^{l}\right)$ in order to fulfill the requirements of proposition 2.1. 


\section{The symplectic perspective of the problem}

In order to have a geometrically clearer picture of what is going on, we shall investigate the symplectic counterpart of the problem studied in the previous section. It is well-known that the invariant quotient in algebraic geometry has a very close analogue in symplectic geometry, namely the Marsden-Weinstein quotient. More precisely, assume that a complex, algebraic, linearly reductive group $G$ acts on a projective variety $X$ and the action is linearized in a very ample line bundle $\mathcal{O}_{X}(1) \rightarrow X$. Let $K$ be the maximal compact subgroup of $G$ and denote $m: X \rightarrow \mathfrak{k}^{*}$ the moment map for the $K$-action, which takes values in the dual of the Lie algebra of $K$. Assume also that the $G$-action on $X$ is such that $X^{\mathrm{ss}}=X_{(0)}^{\mathrm{s}}$, that is all the $G$-semi-stable points are stable and have finite stabilizer. Under these assumptions we have the

Result. A point $x \in X$ is semi-stable if and only if $\overline{G x} \cap m^{-1}(0) \neq \emptyset$. Moreover, the inclusion $m^{-1}(0) / K \hookrightarrow X^{\mathrm{ss}} / G$ is a homeomorphism.

Details and further references can be found in [10].

It is clear that the map $\pi: \bar{M}_{g, k}(X, A) \rightarrow \bar{M}_{g, k}$ which associates to a stable map $[(C, \underline{x}, u)]$ its stabilized curve $\left[\left(C^{s t}, \underline{x}^{s t}\right)\right]$ is preserved by the $G$-action. In order to ensure that the space $\bar{M}_{g, k}$ exists, we shall assume that $2 g-2+k>0$. It is also clear that a point $[(C, \underline{x}, u)] \in \bar{M}_{g, k}(X, A)$ is $G$-semi-stable if and only if it is $G$-semi-stable when it is viewed as a point in the fibre $\bar{M}_{g, k}(X, A) \times_{\operatorname{Spec}} \mathbb{C} k_{\left[\left(C^{s t}, x^{s t}\right)\right]}$ of $\pi$, where $k_{\left[\left(C^{s t}, \underline{x}^{s t}\right)\right]}$ denotes the function field of the corresponding point. This remark justifies the following construction: for a quasi-stable curve $C$ of genus $g$, that is a connected and projective curve whose singularities are at worst ordinary double points, let us define

$$
M_{C, k}(X, A):=\left\{\begin{array}{l|l}
u:(C, \underline{x}) \rightarrow X & \begin{array}{l}
(C, \underline{x}, u) \text { is a stable map, } \\
|x|=k, u_{*}[C]=A
\end{array}
\end{array}\right\} .
$$

We should notice at this point that for defining this space we do not divide it out by the reparametrisations of $C$ as we do in the case of stable maps (see definition 1.1), and this choice will eventually allow us to work with honest maps instead of equivalence classes of maps.

Lemma 3.1. $M_{C, k}(X, A)$ has a natural quasi-projective scheme structure.

Proof. We may assume as usual that $X=\mathbb{P}^{r}$. Recall that a map $(C, \underline{x}, u)$ is stable if and only if $L_{(C, \underline{x}, u)}=\omega_{C}\left(x_{1}+\cdots+x_{k}\right) \otimes u^{*} \mathcal{O}_{\mathbb{P}^{r}}(3) \rightarrow C$ is ample. Also, there is an integer $f=f(g, k, r, d)>0$ with the property that $L_{(C, \underline{x}, u)}^{f} \rightarrow C$ is very ample. In this way, any stable map $(C, \underline{x}, u)$ gave rise to an embed$\operatorname{ding} C \rightarrow \mathbb{P}\left(H^{0}\left(C, L_{(C, \underline{x}, u)}^{f}\right)^{\vee}\right)$ into a space isomorphic to $\mathbb{P}^{N}$, where $N+1=$ 
$\operatorname{dim} H^{0}\left(C, L_{C}^{f}\right)$. The ambiguity in the choice of this isomorphism is given by elements in $P G l(N+1)$. In order to define the space $M_{C, k}(X, A)$ we shall use a fixed but otherwise arbitrary stable map $\left(C, \underline{x}_{0}, u_{0}\right)$. Let us consider the fixed embedding

$$
C \stackrel{j_{0}}{\longrightarrow} \mathbb{P}\left(H^{0}\left(C, L_{\left(C, \underline{x}_{0}, u_{0}\right)}^{f}\right)^{\vee}\right)=\mathbb{P}^{N}
$$

defined by the very ample line bundle $\mathcal{O}(1):=L_{\left(C, \underline{x}_{0}, u_{0}\right)}^{f}$ and let $e$ be its degree on $C$. For another stable map $(C, \underline{x}, u)$, the Hilbert polynomial of its graph $\Gamma_{u} \subset$ $C \times \mathbb{P}^{r}$ is

$$
P(n)=\chi\left(\mathcal{O}_{\Gamma_{u}} \otimes \mathcal{O}_{C}(n) \otimes \mathcal{O}_{\mathbb{P}^{r}}(n)\right)=n(e+d)+\chi\left(\Gamma_{u}\right)=n(d+e)+(1-g) .
$$

So each stable map $(C, \underline{x}, u)$ defines a point $\left(\Gamma_{u}, \underline{x}\right) \in \mathcal{H} i l b_{C \times \mathbb{P}^{r}}^{P} \times\left(C \times \mathbb{P}^{r}\right)^{k}$. Using the embedding $j_{0}: C \rightarrow \mathbb{P}^{N}$, the graph $\Gamma_{u}$ can be viewed as a subvariety of $\mathbb{P}^{N} \times \mathbb{P}^{r}=Y$ and its Hilbert polynomial with respect to the very ample line bundle $\mathcal{L}=\mathcal{O}_{\mathbb{P}^{N}}(1) \otimes \mathcal{O}_{\mathbb{P}^{r}}(1) \rightarrow Y$ is $P$ also.

Clearly, the same is true for any closed subscheme $Z$ of $C \times \mathbb{P}^{r}$ : the Hilbert polynomial inside $C \times \mathbb{P}^{r}$ with respect to $\mathcal{O}_{C}(1) \otimes \mathcal{O}_{\mathbb{P}^{r}}(1)$ is the same as the Hilbert polynomial of its image $j_{0 *} Z$ inside $Y$ with respect to $\mathcal{L}$. So we obtain a closed immersion

$$
\mathcal{H}_{C}:=\mathcal{H} i l b_{C \times \mathbb{P}^{r}}^{P} \longrightarrow \mathcal{H} i l b_{Y}^{P}=\mathcal{H}
$$

The ample line on $\mathcal{H}$ is $\operatorname{det} \mathcal{Q} \rightarrow \mathcal{H}$, where $\mathcal{Q}$ is the universal quotient bundle on some Grassmann variety (see section 1). Remember that there is a subscheme $\mathcal{S}$ of $\mathcal{H} \times Y^{k}$ corresponding to the locus of $k$-marked, genus $g$ stable maps to $\mathbb{P}^{r}$ which represent $d$ times the generator of $H_{2}\left(\mathbb{P}^{r}, \mathbb{Z}\right)$. Let consider the commuting diagram

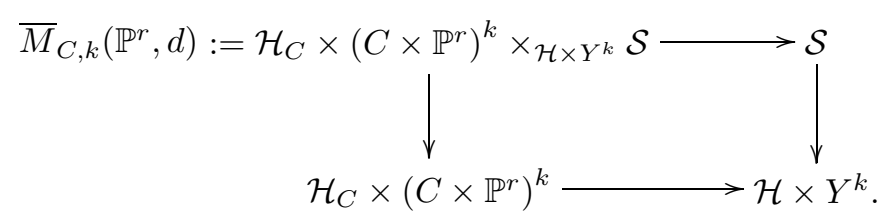

Since $\bar{M}_{C, k}\left(\mathbb{P}^{r}, d\right) \rightarrow \mathcal{H}_{C} \times\left(C \times \mathbb{P}^{r}\right)^{k}$ is an immersion, $\bar{M}_{C, k}\left(\mathbb{P}^{r}, d\right)$ is a quasiprojective scheme. Its ample line bundle is determined by the restriction to $\mathcal{S}$ of $\operatorname{det} \mathcal{Q}_{k} \rightarrow \mathcal{H} \times Y^{k}$. The space $M_{C, k}\left(\mathbb{P}^{r}, d\right)$ is quasi-projective, being an open subset of $\bar{M}_{C, k}\left(\mathbb{P}^{r}, d\right)$.

Remark 3.2. $\bar{M}_{C, k}(X, A)$ is the Gromov compactification of $M_{C, k}(X, A)$. It is also projective if $\left(C, \underline{x}_{0}\right)$ is a stable curve in the sense of Deligne-Mumford (remember that we have used a fixed stable map $\left(C, \underline{x}_{0}, u_{0}\right)$ for the embedding $\left.j_{0}: C \rightarrow \mathbb{P}^{N}\right)$. In this case the intersection of the $P G l(N+1)$-orbit of a stable map in $\mathcal{S} \rightarrow \mathcal{H} \times Y^{k}$ with the image of $\mathcal{H}_{C} \times\left(C \times \mathbb{P}^{r}\right)^{k} \rightarrow \mathcal{H} \times Y^{k}$ consists of finitely many points. Consequently, the $\operatorname{map} \bar{M}_{C, k}\left(\mathbb{P}^{r}, d\right) \rightarrow \bar{M}_{g, k}\left(\mathbb{P}^{r}, d\right)$ is 
generically finite on its image. Because $\bar{M}_{g, k}\left(\mathbb{P}^{r}, d\right)$ is projective, the conclusion follows.

For the symplectic point of view it is convenient to consider $M_{C, k}(X, A)$ with its reduced scheme structure, so that we look at it naively as being a quasi-projective variety. The Zariski tangent space of $M_{C, k}(X, A)$ at a point $(C, \underline{x}, u)$ is

$$
T_{(C, \underline{x}, u)} M_{C, k}(X, A) \subset\left\{\begin{array}{l|l}
\left(s, v_{1}, \ldots, v_{k}\right) & \begin{array}{l}
s \in H^{0}\left(C, u^{*} T X\right) \\
v_{j} \in T_{x_{j}} C, j=1, \ldots, k
\end{array}
\end{array}\right\} .
$$

In the sequel we shall compute the Kähler form on $\bar{M}_{C, k}(X, A)$ induced by its projective embedding. We can see that the ample line bundle $\operatorname{det} \mathcal{Q}_{k} \rightarrow \mathcal{H} \times Y^{k}$ introduced in section 1 and defined by (1.1) is $\operatorname{det}\left(p_{*} \bar{E}^{*} \mathcal{L}^{l}\right) \otimes \overline{\operatorname{ev}}^{*}\left(\mathcal{L}^{l}\right)^{\otimes k}$, where

$$
\begin{array}{cc}
\bar{E}^{*} \mathcal{L}^{l} & \mathcal{L}^{l}=\mathcal{O}_{\mathbb{P}^{N}}(l) \otimes \mathcal{O}_{\mathbb{P}^{r}}(l) \\
\downarrow & \downarrow \\
M_{C, k}(X, A) \times C \stackrel{\bar{E}=\left(j_{0}, E\right)}{\longrightarrow} \mathbb{P}^{N} \times \mathbb{P}^{r}, \quad \text { with } E(u, \zeta):=u(\zeta) \\
p \downarrow \\
M_{C, k}(X, A)
\end{array}
$$

and

$$
\begin{gathered}
M_{C, k}(X, A) \stackrel{\overline{\mathrm{ev}}=\left(\overline{\mathrm{ev}}_{1}, \ldots, \overline{\mathrm{ev}}_{\mathrm{k}}\right)}{\longmapsto}\left(\mathbb{P}^{N} \times \mathbb{P}^{r}\right)^{k}, \quad \overline{\mathrm{ev}}_{\mathrm{j}}=\left(j_{0}, \mathrm{ev}_{\mathrm{j}}\right), \\
(C, \underline{x}, u) \longmapsto\left(\left(j_{0}\left(x_{1}\right), u\left(x_{1}\right)\right), \ldots,\left(j_{0}\left(x_{k}\right), u\left(x_{k}\right)\right)\right) .
\end{gathered}
$$

The Kähler form on $M_{C, k}(X, A)$ induced by its projective embedding is $-1 / 2 \pi i \times$ [curvature of $\left.\operatorname{det}\left(\left(p_{*} \bar{E}^{*} \mathcal{L}^{l}\right) \otimes \overline{\mathrm{ev}}^{*}\left(\mathcal{L}^{l}\right)^{\otimes k}\right)\right]$. For computing this curvature, we need a Hermitian metric on $\mathcal{L}$ and a Kähler metric on the fibres of $M_{C, k}(X, A) \times C \stackrel{p}{\longrightarrow}$ $M_{C, k}(X, A)$ i.e. on $C$. The fibres of $p$ will be all isometric, the Kähler form on them being

$$
\gamma_{C}:=\frac{1}{e} j_{0}^{*} \omega_{\mathbb{P}^{N}}, e:=\operatorname{deg}_{C} \mathcal{O}_{\mathbb{P}^{N}}(1)
$$

This choice reflects the fact that for defining the space $M_{C, k}(X, A)$ we have required the maps $(C, \underline{x}, u)$ to have a fixed domain of definition. On $\mathcal{O}_{\mathbb{P}^{N}}(1)$ and $\mathcal{O}_{\mathbb{P}^{r}}(1)$ consider the Hermitian metrics whose curvatures are $-2 \pi i \omega_{\mathbb{P} N}$ and $-2 \pi i \omega_{\mathbb{P}^{r}}$ respectively, with $\omega_{\mathbb{P} N}$ and $\omega_{\mathbb{P} r}$ the corresponding Fubini-Study forms. There is an induced Hermitian metric on $\mathcal{L}=\mathcal{O}_{\mathbb{P}^{N}}(1) \otimes \mathcal{O}_{\mathbb{P}^{r}}(1)$ and a fortiori on $\bar{E}^{*} \mathcal{L}^{l}$.

It is easy to see which is the expression of the curvature of $\overline{\operatorname{ev}}^{*}\left(\mathcal{L}^{l}\right)^{\bigotimes k}$ at a point $(C, \underline{x}, u) \in M_{C, k}(X, A)$ :

$$
\Omega_{1}:=-\frac{1}{2 \pi i}\left(R^{\mathrm{ev}^{*}\left(\mathcal{L}^{l}\right)^{\otimes k}}\right)_{(C, \underline{x}, u)}=l e \sum_{j=1}^{k}\left(\gamma_{C}\right)_{x_{j}}+l \sum_{j=1}^{k}\left(\operatorname{ev}_{j}^{*} \omega_{\mathbb{P}^{r}}\right)_{(C, \underline{x}, u)} .
$$

For computing the curvature of $\operatorname{det}\left(p_{*} \bar{E}^{*} \mathcal{L}^{l}\right)$ the first thing to notice is that this line bundle is actually the determinant of the derived direct image of $\bar{E}^{*} \mathcal{L}^{l}$, since 
by $p_{*}^{1} \bar{E}^{*} \mathcal{L}^{l}=0$ (see section 1$)$. Consequently, we may apply the differential form of the Atiyah-Singer index theorem for families which is proved in a series of papers [3, 4, 5] by J.-M. Bismut, D. Freed, H. Gillet, Ch. Soulé. According to [5], theorem 0.1 page 51 , if $C$ is a smooth curve, the curvature

$$
\Omega_{2}:=-\frac{1}{2 \pi i} R^{\operatorname{det} p_{*} \bar{E}^{*} \mathcal{L}^{l}}=\int_{C} T d\left(-\frac{1}{2 \pi i} R^{T}\right) \cdot \exp \left(-\frac{1}{2 \pi i} R^{\bar{E}^{*} \mathcal{L}^{l}}\right) .
$$

Here $R^{T_{C}}$ denotes the curvature of the relative tangent bundle of the projection $p$ (i.e. of $T_{C}$ ) corresponding to the Kähler metric $\gamma_{C}$ on $C$ and $R^{\bar{E}^{*} \mathcal{L}^{l}}$ is the curvature of the line bundle $\bar{E}^{*} \mathcal{L}^{l}$ with respect to the Hermitian metric induced by that on $\mathcal{L}^{l}$. Let $\gamma:=(-1 / 2 \pi i) R^{T_{C}}$; it is a real form of type $(1,1)$ on $C$ and therefore $\gamma=h \gamma_{C}$ with $h: C \rightarrow \mathbb{R}$ a smooth function having the property that $\int_{C} h \gamma_{C}=2(1-g)$. On the other hand,

$$
-\frac{1}{2 \pi i} R^{\bar{E}^{*} \mathcal{L}^{l}}=l \bar{E}^{*}\left(-\frac{1}{2 \pi i} R^{\mathcal{L}}\right)=l \bar{E}^{*}\left(\omega_{\mathbb{P} N}+\omega_{\mathbb{P} r}\right)=l\left(e \gamma_{C}+E^{*} \omega_{\mathbb{P} r}\right) .
$$

The form $\Omega_{2}$ is the degree two term in

$$
\int_{C}\left(1+\frac{1}{2} \gamma\right)\left(1+l\left(e \gamma_{C}+E^{*} \omega_{\mathbb{P}^{r}}\right)+\frac{l^{2}}{2}\left(e \gamma_{C}+E^{*} \omega_{\mathbb{P}^{r}}\right)^{2}\right) .
$$

Making the computations we obtain:

$$
\begin{aligned}
\Omega_{2} & =\frac{l^{2}}{2} \int_{C}\left(e \gamma_{C}+E^{*} \omega_{\mathbb{P} r}\right)^{2}+\frac{l}{2} \int_{C}\left(e \gamma_{C}+E^{*} \omega_{\mathbb{P} r}\right) \wedge \gamma \\
& =\frac{l^{2}}{2} \int_{C}\left(E^{*} \omega_{\mathbb{P}^{r}}\right)^{2}+2 e E^{*} \omega_{\mathbb{P}^{r}} \wedge \gamma_{C}+\frac{l}{2} \int_{C} E^{*} \omega_{\mathbb{P}^{r}} \wedge \gamma \\
& =\frac{l^{2}}{2} \int_{C}\left(E^{*} \omega_{\mathbb{P} r}\right)^{2}+l^{2} e \int_{C} E^{*} \omega_{\mathbb{P}^{r}} \wedge \gamma_{C}+\frac{l}{2} \int_{C} h \cdot E^{*} \omega_{\mathbb{P}^{r}} \wedge \gamma_{C} .
\end{aligned}
$$

This proves the

Proposition 3.3. The curvature of the line bundle $\operatorname{det}\left(p_{*} \bar{E}^{*} \mathcal{L}^{l}\right) \otimes \overline{\mathrm{ev}}^{*}\left(\mathcal{L}^{l}\right)^{\otimes k} d i$ vided by $-2 \pi i$ is $\Omega=\Omega_{1}+\Omega_{2}$. It represents the Kähler form on $M_{C, k}(X, A)$ induced by the projective embedding described in lemma 3.1.

Let us come back to the initial set-up: a complex, connected, linear algebraic group $G$ acts on a smooth, irreducible complex projective variety $X$ and the action is linearized in a very ample line bundle $\mathcal{O}_{X}(1) \rightarrow X$. In this case, using the linear system associated to $\mathcal{O}_{X}(1)$, we may assume that $G$ acts on $\mathbb{C}^{r+1}$ and, by an appropriate choice of coordinates, we are allowed to assume that the maximal compact subgroup $K$ of $G$ is included in $U(r+1)$. There is an induced action of $G$ (so, a fortiori, of $K$ ) on $M_{C, k}(X, A)$ defined by: $g \times(C, \underline{x}, u) \mapsto(C, \underline{x}, g u)$. Because the $K$-action preserves the Fubini-Study form $\omega_{\mathbb{p} r}$ and the maps $\bar{E}$ and $\overline{\mathrm{ev}}$ 
are obviously both $K$-equivariant, it follows that $\Omega$ is $K$-invariant also. Our next goal is to determine the moment map corresponding to this Hamiltonian action.

We start by studying the case when $G=\mathbb{C}^{*}$; the induced $S^{1}$-action on $X$ gives rise to a vector field $\xi$ on $X$ having the property that $\mathcal{L}_{\xi} \omega=0$ and $\mathcal{L}_{\xi} J=0$ because $S^{1}$ acts on $X$ by isometries. The vector field $J \xi$ does not preserve $\omega$ in general but still preserves the complex structure of $X$. In fact the vector field $J \xi$ corresponds to the (holomorphic) action of $\mathbb{R}_{+}^{*} \hookrightarrow \mathbb{C}^{*}$. The moment map $m: X \longrightarrow \mathbb{R}$ corresponding to the action is $S^{1}$-invariant and

$$
\mathrm{d} m_{x}\left(J \xi_{x}\right)=\omega\left(\xi_{x}, J \xi_{x}\right)=\left\|\xi_{x}\right\|^{2},
$$

so that $m$ is increasing along the flow lines of $J \xi$.

Let us denote by $\mathcal{V}$ the vector field on $M_{C, k}(X, A)$ determined by the (holomorphic) $S^{1}$-action on $M_{C, k}(X, A)$. At a point $(C, \underline{x}, u)$,

$$
\mathcal{V}_{(C, \underline{x}, u)}=\left(u^{*} \xi, 0, \ldots, 0\right) \in H^{0}\left(C, u^{*} T X\right) \times T_{x_{1}} C \times \ldots T_{x_{k}} C .
$$

Lemma 3.4. The function

$$
\begin{gathered}
\Psi: M_{C, k}(X, A) \longrightarrow \mathbb{R} \\
\Psi:=l^{2} e \int_{C}(m \circ E) \gamma_{C}+l^{2} \int_{C}(m \circ E) E^{*} \omega_{\mathbb{P}^{r}}+\frac{l}{2} \int_{C} h(m \circ E) \gamma_{C}+l \sum_{j=1}^{k} m \circ \mathrm{ev}_{j}
\end{gathered}
$$

is a moment map for the $S^{1}$-action.

Proof. Because $E, \mathrm{ev}_{j}, m, \omega_{\mathrm{p} r}$ are all $S^{1}$-invariant, it follows that $\Psi$ is also. For proving that $\Psi$ is a moment map, we need to show that its differential is the same as the contraction of the Kähler form $\Omega$ on $M_{C, k}(X, A)$ with the vector field $\mathcal{V}$. It what follows, the symbol " $\lrcorner$ " will always denote the contraction of a differential form with a vector field.

The contraction $\mathcal{V}\lrcorner\left(\gamma_{C}\right)_{x_{j}}=0$ because the $T_{x_{1}} C \times \cdots \times T_{x_{k}} C$-component of $\mathcal{V}$ is zero.

$$
\begin{aligned}
\mathcal{V}\lrcorner\left(\mathrm{ev}_{j}^{*} \omega_{\mathbb{P}^{r}}\right) & \left.=\mathrm{ev}_{j}^{*}\left(\left(\mathrm{ev}_{j *} \mathcal{V}\right)\right\lrcorner \omega_{\mathbb{P}^{r}}\right)=\mathrm{ev}_{j}^{*}\left(\xi_{\left.\left.\mathrm{ev}_{j}(\cdot)\right\lrcorner \omega_{\mathbb{P}^{r}}\right)}\right. \\
& =\mathrm{ev}_{j}^{*}\left(\mathrm{~d}_{\mathrm{ev}_{j}(\cdot)}\right)=\mathrm{d}\left(m \circ \mathrm{ev}_{j}\right) . \\
\mathcal{V}\lrcorner \int_{C} E^{*} \omega_{\mathbb{P}^{r}} & \left.\left.\wedge \gamma_{C}=\int_{C} \mathcal{V}\right\lrcorner\left(E^{*} \omega_{\mathbb{P}^{r}} \wedge \gamma_{C}\right)=\int_{C}(\mathcal{V}\lrcorner E^{*} \omega_{\mathbb{P}^{r}}\right) \wedge \gamma_{C} .
\end{aligned}
$$

At a point $p \in C$,

$$
\begin{aligned}
\left.\mathcal{V}_{p}\right\lrcorner\left(E^{*} \omega_{\mathbb{P}^{r}}\right)_{p} & \left.\left.=E^{*}\left(E_{*} \mathcal{V}_{p}\right\lrcorner \omega_{\mathbb{P}^{r}, u(p)}\right)=E^{*}\left(\xi_{u(p)}\right\lrcorner \omega_{\mathbb{P}^{r}, u(p)}\right) \\
& \left.=E^{*}\left(\xi_{u(p)}\right\lrcorner \omega_{\mathbb{P}^{r}, u(p)}\right)=E^{*}\left(\mathrm{~d} m_{u(p)}\right)=\mathrm{d}(m \circ E)_{p} .
\end{aligned}
$$

and consequently

$$
\begin{aligned}
\mathcal{V}\lrcorner \int_{C} E^{*} \omega_{\mathbb{P}^{r}} \wedge \gamma_{C} & =\int_{C} \mathrm{~d}(m \circ E) \wedge \gamma_{C}=\int_{C} \mathrm{~d}\left((m \circ E) \gamma_{C}\right) \\
& =\mathrm{d} \int_{C}(m \circ E) \gamma_{C} .
\end{aligned}
$$




$$
\begin{aligned}
\mathcal{V}\lrcorner \int_{C} h & \left.\cdot E^{*} \omega_{\mathbb{P}^{r}} \wedge \gamma_{C}=\int_{C} \mathcal{V}\right\lrcorner\left(h \cdot E^{*} \omega_{\mathbb{P}^{r}} \wedge \gamma_{C}\right) \\
& \left.=\int_{C} h \cdot(\mathcal{V}\lrcorner E^{*} \omega_{\mathbb{P}^{r}}\right) \wedge \gamma_{C}=\int_{C} h \cdot \mathrm{d}(m \circ E) \wedge \gamma_{C} \\
& \stackrel{(\star)}{=} \int_{C} \mathrm{~d}\left(h(m \circ E) \gamma_{C}\right)=\mathrm{d} \int_{C} h(m \circ E) \gamma_{C} .
\end{aligned}
$$

For writing equality $(\star)$, we have used that $\mathrm{d} h \wedge \gamma_{C}=\mathrm{d}_{C} h \wedge \gamma_{C}=0$.

$$
\begin{aligned}
\mathcal{V}\lrcorner \int_{C}\left(E^{*} \omega_{\mathbb{P} r}\right)^{2} & \left.=2 \int_{C}(\mathcal{V}\lrcorner E^{*} \omega_{\mathbb{P} r}\right) \wedge E^{*} \omega_{\mathbb{P} r}=2 \int_{C} \mathrm{~d}(m \circ E) E^{*} \omega_{\mathbb{P} r} \\
& =2 \int_{C} \mathrm{~d}\left((m \circ E) E^{*} \omega_{\mathbb{P} r}\right)=\mathrm{d}\left(2 \int_{C}(m \circ E) E^{*} \omega_{\mathbb{P} r}\right)
\end{aligned}
$$

All together, these equalities show that $\Psi$ is indeed a moment map.

Using the previous computations, one can derive easily the formula of the moment map on $M_{C, k}(X, A)$ for general group actions.

Proposition 3.5. Let $m: X \rightarrow \mathfrak{k}^{*}$ be the moment map for the K-action on $X$. Then the function

$$
\begin{gathered}
\Psi: M_{C, k}(X, A) \rightarrow \mathfrak{k}^{*} \\
\Psi:=l^{2} e \int_{C}(m \circ E) \gamma_{C}+l^{2} \int_{C}(m \circ E) E^{*} \omega_{\mathbb{P}^{r}}+\frac{l}{2} \int_{C} h(m \circ E) \gamma_{C}+l \sum_{j=1}^{k} m \circ \mathrm{ev}_{j}
\end{gathered}
$$

is the moment map for the $K$-action on $M_{C, k}(X, A)$.

Proof. Because $E, \mathrm{ev}_{j}, \omega_{\mathbb{p}^{r}}$ are all $K$-invariant, and

$$
m(a \cdot x)=\operatorname{Ad}\left(a^{-1}\right) \cdot m(x), \quad \forall x \in X, \forall a \in K,
$$

it follows that this latter property holds for $\Psi$ also. In order to check that the formula gives indeed a moment map, we must show that

$$
\left.(\mathrm{d} \Psi, a)=\mathcal{V}_{a}\right\lrcorner \Omega, \quad \forall a \in \mathfrak{k} .
$$

Here $\mathcal{V}_{a}$ stays for the vector field on $M_{C, k}(X, A)$ induced by the infinitesimal action of $a$, and $($,$) denotes the natural pairing between \mathfrak{k}$ and its dual. Since this relation is linear in $a$ on one hand, and $\mathfrak{k}$ is generated as a vector space by the tangent vectors $a_{\lambda}:=\left.\frac{\mathrm{d}}{\mathrm{d} \theta} \lambda\left(e^{i \theta}\right)\right|_{\theta=0}$ for $\lambda: S^{1} \rightarrow K$ a 1-PS on the other hand, it is enough to check the equality above for such $a_{\lambda}$ 's.

For a fixed one parameter subgroup $\lambda$, the pairing $\left(\Psi, a_{\lambda}\right)$ is given by the same formula as $\Psi$, except that $m$ is replaced with $m_{\lambda}:=\left(m, a_{\lambda}\right)$; but the function $m_{\lambda}$ defined in this way is nothing but the moment map corresponding to the induced $S^{1}$-action on $X$, and the lemma 3.4 implies that $\left(\Psi, a_{\lambda}\right)$ equals the moment map $\Psi_{\lambda}$ on $M_{C, k}(X, A)$ corresponding to the induced $S^{1}$-action. But for this one we do know already that $\left.\mathrm{d} \Psi_{\lambda}=\mathcal{V}_{a_{\lambda}}\right\lrcorner \Omega$. 
Remark 3.6. It is interesting to study the moment map for large $e$ and $l$. We should recall that $e$ is defined in (3.1) as the degree of a certain very ample line bundle on $C$ used to get a fixed embedding of $C$ into a projective space, while $l$ is an integer large enough for obtaining a projective embedding of the Hilbert scheme $\mathcal{H}$ (actually $l$ does depend on $e$ ). Because $X$ is compact, the moment map $m$ is bounded and therefore the last two terms in $\frac{1}{e l^{2}} \Psi$ are of order $O\left(\frac{1}{e l}\right)$. We will show that the first term is of order $O\left(\frac{1}{e}\right)$. Indeed, since maps $u \in M_{C, k}(X, A)$ are holomorphic, the pull-back $u^{*} \omega_{\mathbb{P} r} \geq 0$ as a form on $C$. This implies that

$$
\left|\frac{1}{e} \int_{C}(m \circ u) u^{*} \omega_{\mathbb{P}^{r}}\right| \leq \frac{\max _{X}|m|}{e} \int_{C} u^{*} \omega_{\mathbb{P}^{r}}=\frac{d \max _{X}|m|}{e}=O\left(\frac{1}{e}\right),
$$

where $d$ denotes, as usual, the degree of the composite map $C \rightarrow X \rightarrow \mathbb{P}^{r}$, which is a constant.

The conclusion of this discussion is that for large $e$ and $l$

$$
\frac{1}{e l^{2}} \Psi \sim \int_{C}(m \circ E) \gamma_{C},
$$

so the zero set of $\Psi$ will be close to the zeros of this second function. Notice that the right hand side of (3.3) is the moment map corresponding to the Kähler form

$$
\Omega_{\infty}:=\int_{C} E^{*} \omega_{\mathbb{P}^{r}} \wedge \gamma_{C}
$$

on $M_{C, k}(X, A)$.

At this point some care is required because $\gamma_{C}$, as it is defined by (3.2), does depend on $e$. However, it is well-known (see [16]) that the sequence of such metrics converges to a metric on $C$, which was still denoted $\gamma_{C}$.

Recall that the smooth real-valued function $h$ defined on $C$ is the "quotient" $R^{T_{C}} / \gamma_{C}$, where $R^{T_{C}}$ denotes the curvature of the tangent bundle of $C$ with respect to the Kähler form $\gamma_{C}$. This last form was defined in terms of a fixed projective embedding of $C$; in particular, it does not depend on $l$. For obtaining the projective embedding of the Hilbert scheme we had to take large positive integral values for $l$, and therefore we may assume that $l e+\frac{1}{2} h$ is a strictly positive function on $C$. Notice also that since $u$ is holomorphic and $\omega_{\mathbb{p} r}$ is a positive $(1,1)$-form, the $(1,1)$-form $u^{*} \omega_{\mathbb{P}^{r}}$ on $C$ is still positive. Now we recover easily the

Theorem 3.7 (2.5). A stable map $(C, \underline{x}, u)$, with $C$ smooth, having the property that Image $u \subset X_{(0)}^{\mathrm{s}}\left(\mathcal{O}_{X}(1)\right)$, defines a $G$-stable point in $\bar{M}_{g, k}(X, A)$.

Proof. According to the Hilbert-Mumford criterion, it is sufficient to prove the statement for every 1-PS $\lambda: \mathbb{C}^{*} \rightarrow G$. For a fixed 1-PS $\lambda$ of $G$, the point $(C, \underline{x}, u)$ is $\lambda$-stable, if its $\mathbb{C}^{*}$-orbit meets the zero-level set of the function $\Psi_{\lambda}$ on $M_{C, k}(X, A)$. Assume for instance that $\Psi_{\lambda}(C, \underline{x}, u)<0$. By hypothesis, Image $u \subset X_{(0)}^{\mathrm{s}} \subset X^{\mathrm{s}(\lambda)}$, so that under the $\mathbb{R}_{+}^{*}$-action all the points $u(p), p \in C$, meet the $m_{\lambda}^{-1}(0)$-level. 
As $C$ is compact, we find $r \gg 0$, which depends on $u$, with the property that $m_{\lambda}(\lambda(r) u(p))>0$ for all $p \in C$. Since

$$
\Psi_{\lambda}(C, \underline{x}, u)=l \int_{C}\left(l e+\frac{1}{2} h\right)\left(m_{\lambda} \circ u\right) \gamma_{C}+l^{2} \int_{C}\left(m_{\lambda} \circ u\right) u^{*} \omega_{\mathbb{P}^{r}}+l \sum_{j=1}^{k} m_{\lambda}\left(u\left(x_{j}\right)\right)
$$

for such a large $r$, the translated map $\lambda(r) u$ has the property that $\Psi_{\lambda}(C, \underline{x}, \lambda(r) u)>$ 0 . A continuity argument proves that there is (a unique) $r_{0}$ with $\left(C, \underline{x}, r_{0} u\right) \in$ $\Psi_{\lambda}^{-1}(0)$. It is also clear from the hypothesis that $(C, \underline{x}, u)$ has finite stabilizer.

For $G=\mathbb{C}^{*}$ we can improve the result above, namely if $u:(C, \underline{x}) \rightarrow X$ is a morphism whose image is not contained in the unstable locus of $X$, then it defines a stable point in $M_{C, k}(X, A)$ for a suitable linearization of the action. In view of corollary 2.4 , this result is optimal and completely characterizes the $\mathbb{C}^{*}$-stable points of $M_{C, k}(X, A)$ : a point $(C, \underline{x}, u)$ is stable if and only if $u(C)$ is not contained in the $\mathbb{C}^{*}$-unstable locus of $X$.

Let us prove this remark: we are going to show that for such a morphism $u$, $\lim _{r \rightarrow+\infty} \Psi(r u)>0$ and $\lim _{r \rightarrow-\infty} \Psi(r u)<0$. Since $X^{\mathrm{ss}}=X_{(0)}^{\mathrm{s}}, 0 \in \mathbb{R}$ is a regular value of the moment map $m: X \rightarrow \mathbb{R}$, and there exists an $\eta>0$ such that any $\rho \in(-\eta, \eta)$ is regular for $m$. Consider a relatively compact open subset $U_{\varepsilon} \Subset C$ such that $u\left(U_{\varepsilon}\right) \subset X_{(0)}^{\mathrm{s}}, 1-\varepsilon \leq \int_{U_{\varepsilon}} \gamma_{C} \leq 1$ with

$$
0<\varepsilon<\frac{\eta}{4\left(1+\max _{X}|m|\right)}
$$

Since $u\left(U_{\varepsilon}\right) \subset X_{(0)}^{\mathrm{s}}$, we deduce that for $r \gg 0, m(r u(p))>\eta / 2$ for all $p \in \bar{U}_{\varepsilon}$. Writing $C=\bar{U}_{\varepsilon} \cup\left(C \backslash U_{\varepsilon}\right)$, we obtain that for such a large $r$,

$$
\begin{aligned}
\frac{1}{l^{2} e} \Psi(r u) & =\int_{C}(m \circ r u) \gamma_{C}+\frac{1}{e} \int_{C}(m \circ r u)(r u)^{*} \omega_{\mathbb{P}^{r}} \\
& +\frac{1}{2 l e} \int_{C} h(m \circ r u) \gamma_{C}+\frac{1}{l e} \sum_{j=1}^{k} m\left(r u\left(x_{j}\right)\right) \\
& \geq \frac{\eta}{2}(1-\varepsilon)-\varepsilon \max _{X}|m|-O\left(\frac{\max _{X}|m|}{e}\right) \\
& =\frac{\eta}{2}-\varepsilon\left(1+\max _{X}|m|\right)-O\left(\frac{\max _{X}|m|}{e}\right) .
\end{aligned}
$$

We can see that for $e$ large enough, that is for suitable choice of the linearization for the $S^{1}$-action on $M_{C, k}(X, A), \Psi(r u)$ is positive. A similar study applies in the $r \rightarrow-\infty$ limit. 


\section{First application: comparison of invariants}

In this section we shall use the results obtained so far, for comparing the genus zero Gromov-Witten invariants of a projective manifold with those of its invariant quotient for a group action. Before stating our result we introduce the following

Definition 4.1. Let $f: M \rightarrow N$ be a rational map. We define $f^{*}: H^{*}(N) \rightarrow$ $H^{*}(M)$ by $f^{*} \alpha:=\mathrm{PD}_{M}\left(p_{*}\left(\Gamma_{f} \cap q^{*} \alpha\right)\right)$, where $\Gamma_{f} \subset M \times N$ denotes the closure of the graph of $f$ with the projections $p$ and $q$ on $M$ and $N$ respectively and PD stands for Poincaré-duality.

This is just another way to express the correspondence induced by $f$, and we should keep in mind that $f^{*}$ is not a ring homomorphism in general.

Theorem 4.2. Consider a complex, connected, linearly reductive group $G$ acting on the irreducible projective variety $X$, and also a linearization of the action in a very ample line bundle $\mathcal{O}_{X}(1) \rightarrow X$. Denote by $\zeta \in H^{2} \operatorname{dim} G(X ; \mathbb{Q})$ the class of a rational transverse slice to $X \rightarrow \hat{X}$. Let $A \in H_{2}(X ; \mathbb{Z})$ be a class which can be represented by a morphism $\mathbb{P}^{1} \rightarrow X^{\mathrm{ss}}$ and denote $\hat{A}$ the class of its image in $\hat{X}$.

Suppose that the following conditions are satisfied:

(a1) $G$ acts freely on the $G$-semi-stable locus of $X$, so that the quotient map $X^{\mathrm{ss}} \rightarrow X / / G=: \hat{X}$ is a principal $G$-bundle (according to [12], Corollaire 1);

(a2) $\bar{M}_{0, k}(X, A)$ is generically smooth and has the expected dimension;

- In the case when $G$ is a complex torus, assume moreover that:

(a3) every irreducible component of $\bar{M}_{0, k}(X, A)$ contains a point represented by a morphism $\mathbb{P}^{1} \rightarrow X^{\mathrm{ss}}$

(a4) $M_{0, k}(\hat{X}, \hat{A}) \subset \bar{M}_{0, k}(\hat{X}, \hat{A})$ is a dense open subset.

- For general reductive $G$, assume that both $\bar{M}_{0, k}(X, A)$ and $\bar{M}_{0, k}(\hat{X}, \hat{A})$ are irreducible.

Then for any $\hat{\alpha} \in H^{*}\left(\hat{X}^{k} ; \mathbb{Q}\right)$ the following equality between the genus zero Gromov-Witten invariants holds:

$$
G W_{\hat{X}, \hat{A}}^{0, k}(\hat{\alpha})=G W_{X, A}^{0, k}\left(\alpha \cup\left(p r_{X}^{X^{k}}\right)^{*} \zeta\right),
$$

where $\alpha \in H^{*}\left(X^{k} ; \mathbb{Q}\right)$ is obtained from $\hat{\alpha}$ using the correspondence induced by the rational map $X^{k} \rightarrow \hat{X}^{k}$, and $\operatorname{pr}_{X}^{X^{k}}: X^{k} \rightarrow X$ denotes the projection onto the first component.

Before proceeding to the proof of the theorem, I would like to discuss

When are the hypothesis in theorem 4.2 satisfied? The condition (a1) on $G$ to act freely on the semi-stable locus of $X$ is necessary in order to ensure the equality of the expected dimensions of the spaces of stable maps involved inhere. It is unlikely to have any relations between the invariants if there are semi-stable points in $X$ with positive dimensional stabilizers. I have imposed the condition (a2) in order to avoid the use of the virtual class, which could be a rather difficult task in 
the present context. Condition (a3) excludes the existence of irreducible components of $\bar{M}_{0, k}(X, A)$ such that the images of all the corresponding morphisms cut the unstable locus of $X$. Condition (a4) says that there should be no irreducible component of $\bar{M}_{0, k}(\hat{X}, \hat{A})$ such that all its points represent stable maps whose domain of definition are trees of $\mathbb{P}^{1}$ 's.

I would like now to enumerate some cases where the theorem above applies.

Lemma 4.3. Assuming (a1), the requirements of 4.2 are satisfied in the following cases:

(i) if $G$ is a torus, when $X$ is a convex variety and $T_{X^{\mathrm{ss}}}$ is generated by $G$ invariant sections or

(ii) for general $G$, when both $X$ and $\hat{X}$ are homogeneous varieties.

We should recall that a projective variety $X$ is called convex if for any morphism $v: \mathbb{P}^{1} \rightarrow X, H^{1}\left(\mathbb{P}^{1}, v^{*} T_{X}\right)=0$. Standard examples of such varieties are those whose tangent bundle is globally generated; homogeneous varieties are very special instances of such objects. Theorem 2 in [9] says that for convex varieties, the moduli spaces $\bar{M}_{0, k}(X, A)$ are generically smooth, and the singularities are of finite quotient type.

Proof. (i) The first remark is that in these conditions $\hat{X}$ is a convex variety too: this follows from the exact sequence

$$
0 \longrightarrow \mathcal{O}(\mathcal{L} i e G) \longrightarrow T_{X^{\mathrm{ss}}}^{\mathrm{inv}} \longrightarrow T_{\hat{X}} \longrightarrow 0
$$

on $\hat{X}$ associated to the principal bundle $X^{\mathrm{ss}} \rightarrow \hat{X}$.

Since both $X$ and $\hat{X}$ are convex, $M_{0, k}(X, A) \subset \bar{M}_{0, k}(X, A)$ and $M_{0, k}(\hat{X}, \hat{A}) \subset$ $\bar{M}_{0, k}(\hat{X}, \hat{A})$ are open and dense (see [9], theorem 2, page 56), so that (a4) is fullfilled; convexity implies also that we are working in the expected dimension. The only thing to check is condition (a3): since $M_{0, k}(X, A) \subset \bar{M}_{0, k}(X, A)$ is dense, we may restrict our attention to stable maps whose domain is $\mathbb{P}^{1}$. Since the evaluation map ev at the $(k+1)^{\text {th }}$ marked point on $M_{0, k+1}(X, A)$ is submersive, any morphism $\mathbb{P}^{1} \rightarrow X$ can be 'pulled away' from the unstable locus of $X$. Indeed, if $X^{\text {unstable }} \hookrightarrow X$ has codimension at least two, the inverse image $\mathrm{ev}^{-1}\left(X^{\text {unstable }}\right) \hookrightarrow \bar{M}_{0, k+1}(X, A)$ has the same property since ev is submersive, so the direct image of this set has codimension at least one in $\bar{M}_{0, k}(X, A)$. In the general case, let us write $X^{\text {unstable }}=\Delta \cup Z$, with $\Delta=\Delta_{1} \cup \cdots \cup \Delta_{s}$ a union of irreducible divisors and $Z \hookrightarrow X$ of codimension at least two. We have seen that this later component does not cause any trouble, so let us study the first one. The assumption on the class $A$ implies that $A \cdot \Delta_{\sigma}=0, \forall \sigma=1, \ldots, s$. Let us focus now on an irreducible component of $\bar{M}_{0, k}(X, A)$ : since $X$ is convex, $M_{0, k}(X, A) \subset \bar{M}_{0, k}(X, A)$ is dense, and therefore it certainly contains a morphism $u: \mathbb{P}^{1} \rightarrow X$ representing $A$, but it might happen that its image is contained in $\Delta$. The submersivity of the evaluation map implies that we can deform $u$ into a $u^{\prime}$ whose image is not contained in $\Delta$, and is also disjoint from $Z$. But in this case 
$u^{\prime}\left(\mathbb{P}^{1}\right) \cdot \Delta_{\sigma} \geq 0$, with equality if and only if $u^{\prime}\left(\mathbb{P}^{1}\right) \cap \Delta_{\sigma}=\emptyset$ for all $\sigma=1, \ldots, s$; since $A \cdot \Delta_{\sigma}=0$, we deduce that $u^{\prime}$ is disjoint from $X^{\text {unstable }}$.

(ii) The statement is the content of the article [17].

Let us return now to the proof of theorem 4.2, and consider the following commutative diagram:

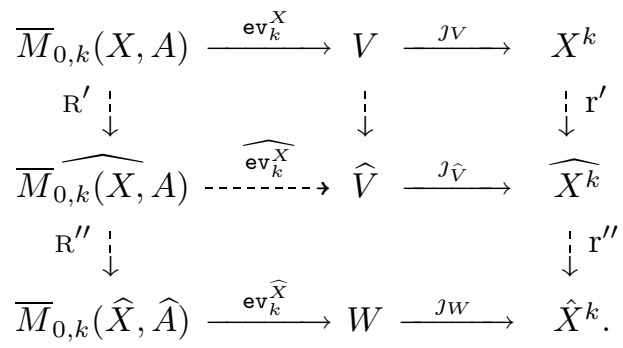

The notations are as follows: $V$ and $W$ are respectively the images of the morphisms $\mathrm{ev}_{k}^{X}$ and $\mathrm{ev}_{k}^{\widehat{X}}$, both with the reduced scheme structure. The group $G$ acts on $X^{k}$ in a diagonal fashion and the evaluation morphism $\mathrm{ev}_{k}^{X}$ is $G$-equivariant. The invariant quotients of $\bar{M}_{g, k}(X, A), V$ and $X^{k}$ are denoted respectively $\bar{M}_{g, k}(X, A), \widehat{V}$ and $\widehat{X^{k}}$. Notice that $\widehat{V} \neq \emptyset$ as soon as there are stable maps whose image is contained in the $G$-semi-stable locus of $X$. The universality property of quotients implies the existence of the rational map $\widehat{\mathrm{ev}_{k}^{X}}$. The quotient map $X^{k} \rightarrow \hat{X}^{k}$ naturally factorizes through a rational map $\mathrm{r}^{\prime \prime}: \widehat{X^{k}} \rightarrow \hat{X}^{k}$ whose general fibre is isomorphic to $G^{k} / G$. If $G$ was a torus, then $\mathrm{r}^{\prime \prime}$ would have been the quotient map for the induced $G^{k} / G$-action on $\widehat{X^{k}}$.

Proposition 4.4. Under the assumptions of theorem 4.2, the map

$$
\mathrm{R}^{\prime \prime}: \bar{M}_{0, k}(X, A) / / G \rightarrow \bar{M}_{0, k}(\hat{X}, \hat{A})
$$

is birational.

Proof. We start noticing that $\mathrm{R}^{\prime \prime}$ is generically injective on its image. Indeed, we may restrict our attention to maps whose image is contained in $X^{\mathrm{ss}}$ : for $G$ a torus, this is just the assumption (a3), while for general $G$, it follows from the assumption of the irreducibility of the space of stable maps and the assumed existence of one such map. If $u_{1}, u_{2}: \mathbb{P}^{1} \rightarrow X^{\mathrm{ss}}$ are morphisms such that $\phi \circ u_{1}=\phi \circ u_{2}$, it follows from (a1) that there is a morphism $g: \mathbb{P}^{1} \rightarrow G$ such that $u_{2}(\zeta)=g(\zeta) u_{1}(\zeta)$ for all $\zeta \in \mathbb{P}^{1}$. Since $G$ is affine, the morphism $g$ must be constant and therefore $u_{1}$ and $u_{2}$ represent the same point in $\bar{M}_{0, k}(X, A) / / G$.

Write

$$
\bar{M}_{0, k}(X, A)=\bigcup_{\nu} \bar{M}_{0, k}(X, A)_{\nu}
$$


as the union of its irreducible components. Assumption (a3) says that each component $\bar{M}_{0, k}(X, A)_{\nu}$ contains a non-empty open subset $\bar{M}_{0, k}(X, A)_{\nu}^{o}$ having the property that its points represent stable maps defined on $\mathbb{P}^{1}$, with image completely contained in the $G$-semi-stable locus of $X$.

We know already that the maps $\mathrm{R}_{\nu}^{\prime \prime}: M_{0, k}(X, A)_{\nu}^{o} \rightarrow \bar{M}_{0, k}(\hat{X}, \hat{A})$ are birational on their image; let us denote $\bar{M}_{0, k}(\hat{X}, \hat{A})_{\nu}$ the closures of these images. They are distinct irreducible components of $\bar{M}_{0, k}(\hat{X}, \hat{A})$, each of them having expected dimension. In fact, for $\left[\left(\mathbb{P}^{1}, \underline{x}, u\right)\right] \in \bar{M}_{0, k}(X, A)_{\nu}^{o}$ the composite $\left[\left(\mathbb{P}^{1}, \underline{x}, \hat{u}\right)\right] \in$ $\bar{M}_{0, k}(\hat{X}, \hat{A})_{\nu}$ is a smooth point of $\bar{M}_{0, k}(\hat{X}, \hat{A})$; this can be seen pulling back by $u$ the exact sequence

$$
0 \longrightarrow T_{\mathfrak{g}} \longrightarrow T_{X \mathrm{ss}} \longrightarrow \phi^{*} T_{\hat{X}} \longrightarrow 0,
$$

on $X$, where $T_{\mathfrak{g}}$ denoted the trivial sub-bundle generated by the infinitesimal action of $G$.

For proving that $\mathrm{R}^{\prime \prime}$ is dominant, we have to show that if $\bar{M}_{0, k}(\hat{X}, \hat{A})_{\mu}$ is an irreducible component of $\bar{M}_{0, k}(\hat{X}, \hat{A})$, then $\mu$ is one of the $\nu$ 's coming from $X$.

Let us start with the case when $G$ is a torus: $\phi: X^{\mathrm{ss}} \rightarrow \hat{X}$ is a principal bundle, and therefore defines a class $\gamma \in \check{H}^{1}\left(\hat{X} ; \mathcal{O}_{\hat{X}}(G)\right)$. The Chern homomorphism defines the class

$$
\operatorname{ch}(\gamma)=\left(\delta_{1}, \ldots, \delta_{r}\right) \in H^{2}(\hat{X} ; \mathbb{Z})^{\oplus r}, \quad \text { with } r=\operatorname{rk}(G)
$$

The assumption (a4) says that it is enough to restrict ourselves to morphisms $\hat{u}: \mathbb{P}^{1} \rightarrow \hat{X}$ : the topological type of the principal bundle $\hat{u}^{*} X^{\mathrm{ss}} \rightarrow \mathbb{P}^{1}$ is given by the $r$-tuple of integers

$$
\left(\left\langle\delta_{1}, \hat{A}\right\rangle, \ldots,\left\langle\delta_{r}, \hat{A}\right\rangle\right)=(0, \ldots, 0)
$$

The last equality holds because the class $\hat{A}$ is induced by the class $A \in H^{2}\left(X^{\mathrm{ss}} ; \mathbb{Z}\right)$. Since $\operatorname{Pic}^{0}\left(\mathbb{P}^{1}\right)=\{1\}$, we deduce that $\hat{u}^{*} X^{\mathrm{ss}} \rightarrow \mathbb{P}^{1}$ is a holomorphically trivial torus bundle, and consequently it has a section. This in turn implies that there is a morphism $u: \mathbb{P}^{1} \rightarrow X^{\mathrm{ss}}$ such that $\hat{u}=\phi \circ u$.

When $G$ is an arbitrary reductive group, let us consider the principal bundle

$$
\mathrm{ev}^{*} X^{\mathrm{ss}} \longrightarrow M_{0, k+1}(\hat{X}, \hat{A}),
$$

where, as before, ev stays for the evaluation at the $(k+1)^{\text {th }}$ marked point. We know that there is a morphism $u_{0}: \mathbb{P}^{1} \rightarrow X^{\mathrm{ss}}$ representing the class $A$; for the composed map $\hat{u}_{0}:=\phi \circ u_{0}$ this means that the principal bundle $\hat{u}_{0}^{*} X^{\mathrm{ss}} \rightarrow \mathbb{P}^{1}$ has a section, and consequently is holomorphically trivial. We shall look now at (4.3) as being a family of principal bundles over $\mathbb{P}^{1}$ parameterized by $M_{0, k}(X, A)$ having the property that the points corresponding to $\hat{u}_{0}$ are trivial principal bundles. According to [15], theorem 4.2, the infinitesimal deformations of the trivial principal bundle $P_{0} \rightarrow \mathbb{P}^{1}$ are parameterized by

$$
H^{1}\left(\mathbb{P}^{1}, P_{0} \times \text { Ad } \mathfrak{g}\right)=H^{1}\left(\mathbb{P}^{1}, \mathcal{O}_{\mathbb{P}^{1}}\right)^{\oplus \operatorname{dim} G}=0, \quad \text { where } \mathfrak{g}=\operatorname{Lie}(G) .
$$


This means that the trivial bundle over $\mathbb{P}^{1}$ is rigid, so that there is a whole Zariski open subset $\mathscr{U} \subset M_{0, k}(\hat{X}, \hat{A})$ having the property that for any $v \in \mathscr{U}$, $v^{*} X^{\mathrm{ss}} \rightarrow \mathbb{P}^{1}$ is holomorphically trivial. Since our assumption is that $\bar{M}_{0, k}(\hat{X}, \hat{A})$ is irreducible, we deduce that $\mathscr{U}$ is dense, and we can repeat word-by-word the previous argument. We should notice again that the exact sequence (4.2) implies that $\bar{M}_{0, k}(\hat{X}, \hat{A})$ is generically smooth and has the expected dimension.

In both cases we must check that if $v \in M_{0, k}(\hat{X}, \hat{A})$ has the property that the fibration $v^{*} X^{\mathrm{ss}} \rightarrow \mathbb{P}^{1}$ is trivial and $u$ is a section, then indeed $u_{*}\left[\mathbb{P}^{1}\right]=A$. This can be seen as follows: denoting EG $\rightarrow$ BG the 'universal' $G$-fibration, we have the diagram

$$
\begin{gathered}
H_{2}(\hat{X}) \cong H_{2}\left(\mathrm{EG} \times_{G} X^{\mathrm{ss}}\right) \\
\downarrow^{c} \\
0 \longrightarrow H_{2}(X) \longrightarrow H_{2}\left(\mathrm{EG} \times_{G} X\right) \longrightarrow H_{2}(\mathrm{BG}) \longrightarrow 0,
\end{gathered}
$$

and the class $\hat{A}$ is mapped into $0 \in H_{2}(\mathrm{BG})$, since the fibration $v^{*} X^{\mathrm{ss}} \rightarrow \mathbb{P}^{1}$ is (topologically) trivial. The lower sequence is exact because BG is simply connected, and consequently the class $c(\hat{A}) \in H_{2}\left(\mathrm{EG} \times_{G} X\right)$ actually lives in $H_{2}(X)$. Since the homomorphism $c$ is canonical, and for the morphism $u_{0}: \mathbb{P}^{1} \rightarrow X^{\mathrm{ss}} \subset X$ we know that $u_{0 *}\left[\mathbb{P}^{1}\right]=A$, we deduce that $c(\hat{A})=A$.

For the proof of theorem 4.2, we remark that if $V \subset M$ and $W \subset N$ are complete, irreducible subvarieties such that $V \cap \operatorname{Dom}(f) \neq \emptyset$ and the restriction $f_{V}: V \rightarrow W$ is dominant, then for any $\alpha \in H^{*}(N)$

$$
\left\langle f^{*} \alpha,[V]\right\rangle=\operatorname{deg}\left(f_{V}\right)\langle\alpha,[W]\rangle
$$

where we set $\operatorname{deg}\left(f_{V}\right)=0$ when $\operatorname{dim} V>\operatorname{dim} W$. This claim follows from the fact that $(V \times N) \cdot \Gamma_{f}=\Gamma_{f_{V}}$.

Proof (of theorem 4.2). We have seen in proposition 4.4 that

$$
\bar{M}_{0, k}(X, A)=\bigcup_{\nu} \bar{M}_{0, k}(X, A)_{\nu}, \quad \bar{M}_{0, k}(X, A) / / G=\bigcup_{\nu} \bar{M}_{0, k}(X, A)_{\nu} / / G
$$

and

$$
\bar{M}_{0, k}(\hat{X}, \hat{A})=\bigcup_{\nu} \bar{M}_{0, k}(\hat{X}, \hat{A})_{\nu}
$$

Moreover, $\mathrm{R}_{\nu}^{\prime \prime}: \bar{M}_{0, k}(X, A)_{\nu} / / G \rightarrow \bar{M}_{0, k}(\hat{X}, \hat{A})_{\nu}$ are birational for all $\nu$. Let $V_{\nu} \subset X^{k}$ and $W_{\nu} \subset \hat{X}^{k}$ be respectively the images of the $k$-point evaluation maps on $\bar{M}_{0, k}(X, A)_{\nu}$ and $\bar{M}_{0, k}(\hat{X}, \hat{A})_{\nu}$. Then $V_{\nu} / / G$ is the closure of the image of the evaluation map on $\bar{M}_{0, k}(X, A)_{\nu} / / G$ and there is a natural map $\mathrm{r}_{\nu}^{\prime \prime}: V_{\nu} / / G \rightarrow W_{\nu}$ compatible with the other arrows in (4.1) which is dominant.

The class $\zeta$ which appears in the statement of the theorem is just the class of a "rational section" of the quotient $\phi$, that is $\zeta:=\frac{1}{d} Z$ for a general complete 
intersection $Z \hookrightarrow X$ which transversally intersects, in $d$ points, the closures of the general $G$-orbits in $X$. With this choice for $Z$, the rational map $V_{\nu} \cap\left(Z \times X^{k-1}\right)-\rightarrow$ $V_{\nu} / / G$ is generically finite of degree $d$.

Consider $\hat{\alpha} \in H^{*}\left(\hat{X}^{k}\right)$ and let $\alpha:=\phi^{*} \hat{\alpha}$ for $\phi: X^{k} \rightarrow \hat{X}^{k}$. The discussion preceding this proof applied to the composite $V_{\nu} \cap\left(Z \times X^{k-1}\right) \stackrel{d: 1}{\rightarrow} V_{\nu} / / G_{\nu}^{\stackrel{\mathrm{r}_{\nu}^{\prime \prime}}{\rightarrow}} W_{\nu}$ shows that

$$
\left\langle\alpha,\left[V_{\nu} \cap\left(Z \times X^{k-1}\right)\right]\right\rangle=d \cdot \operatorname{deg}\left(\mathrm{r}^{\prime \prime}\right)\left\langle\hat{\alpha},\left[W_{\nu}\right]\right\rangle .
$$

We can write therefore,

$$
\begin{aligned}
\int_{\bar{M}_{0, k}(\hat{X}, \hat{A})_{\nu}}\left(\mathrm{ev}_{k}^{\hat{X}}\right)^{*} \hat{\alpha}=\operatorname{deg}\left(\mathrm{ev}_{k}^{\hat{X}}\right)\left\langle\hat{\alpha},\left[W_{\nu}\right]\right\rangle=\frac{\operatorname{deg}\left(\mathrm{ev}_{k}^{\hat{X}}\right)}{d \cdot \operatorname{deg}\left(\mathrm{r}_{\nu}^{\prime \prime}\right)}\left\langle\alpha,\left[V_{\nu} \cap\left(Z \times X^{k-1}\right)\right]\right\rangle \\
=\operatorname{deg}\left(\widehat{\operatorname{ev}_{k, \nu}^{X}}\right)\left\langle\alpha,\left[V_{\nu}\right] \cap\left(\operatorname{pr}_{X}^{X}\right)^{k} \zeta\right\rangle=\operatorname{deg}\left(\operatorname{ev}_{k, \nu}^{X}\right)\left\langle\alpha,\left[V_{\nu}\right] \cap\left(\operatorname{pr}_{X}^{X^{k}}\right)^{*} \zeta\right\rangle \\
=\operatorname{deg}\left(\operatorname{ev}_{k, \nu}^{X}\right)\left\langle\alpha \cup\left(\operatorname{pr}_{X}^{X^{k}}\right)^{*} \zeta,\left[V_{\nu}\right]\right\rangle=\int_{\bar{M}_{0, k}(X, A)_{\nu}}\left(\operatorname{ev}_{k}^{X}\right)^{*}\left(\alpha \cup\left(\operatorname{pr}_{X}^{X^{k}}\right)^{*} \zeta\right) .
\end{aligned}
$$

This finishes the case when $\operatorname{deg}\left(\mathrm{r}_{\nu}^{\prime \prime}\right) \neq 0$. When $\operatorname{deg}\left(\mathrm{r}_{\nu}^{\prime \prime}\right)=0$ (that is $\mathrm{r}^{\prime \prime}$ is not generically finite), both sides are zero. Summing these equalities after $\nu$ we get the conclusion.

Remark 4.5. Let us consider $\hat{\alpha}=\hat{\alpha}_{1} \otimes \cdots \otimes \hat{\alpha}_{k} \in H^{*}\left(\hat{X}^{k} ; \mathbb{Q}\right)$ and denote respectively $\alpha_{j}:=\phi^{*} \hat{\alpha}_{j} \in H^{*}(X ; \mathbb{Q})$. The equality which appears in the theorem reads

$$
G W_{\hat{X}, \hat{A}}^{0, k}\left(\hat{\alpha}_{1} \otimes \ldots \otimes \hat{\alpha}_{k}\right)=G W_{X, A}^{0, k}\left(\zeta \cup \alpha_{1} \otimes \ldots \otimes \alpha_{k}\right),
$$

and this form is somewhat odd since the left-hand-side is symmetric in the $k$ entries (we consider cohomology classes of even degree), while the right-hand-side is not symmetric in an obvious way. This means that we get relations among the enumerative invariants of $X$,

$$
G W_{X, A}^{0, k}\left(\zeta \cup \alpha_{1} \otimes \ldots \otimes \alpha_{j} \otimes \ldots \otimes \alpha_{k}\right)=G W_{X, A}^{0, k}\left(\alpha_{1} \otimes \ldots \otimes \zeta \cup \alpha_{j} \otimes \ldots \otimes \alpha_{k}\right),
$$

for all $j=2, \ldots, k$, and the more complicated the group action, the less apparent are these relations.

Another aspect of the problem is that it is possible to construct rather complicated quotients $\hat{X}$ starting with 'simple' varieties $X$. Using this approach, one may hope to get a better insight to the Gromov-Witten invariants of the quotients.

\subsection{Some examples}

The requirements in theorem 4.2 make its applications rather restricted, but we shall see that there are several cases where the theory does apply, and relates the enumerative invariants of apparently non-related objects. 
4.1.1. For $n_{0}, n_{1}, \ldots, n_{p} \geq 1$ integers and $n:=n_{0}+n_{1}+\cdots+n_{p}$, consider the linearized $\left(\mathbb{C}^{*}\right)^{p}$-action on $\mathbb{P}^{n+p+1}$ given by

$$
\begin{gathered}
\left(\mathbb{C}^{*}\right)^{p} \times \mathbb{C}^{n+p+1} \longrightarrow \mathbb{C}^{n+p+1}, \\
\left(t_{1}, \ldots, t_{p}\right) \times\left(z_{0}, z_{1}, \ldots, z_{p}\right):=\left(t_{1} \ldots t_{p} z_{0}, t_{1}^{-1} z_{1}, \ldots, t_{p}^{-1} z_{p}\right), \text { with } z_{j} \in \mathbb{C}^{n_{j}+1} .
\end{gathered}
$$

The unstable locus of $\mathbb{P}^{n+p}$ is the union $\bigcup_{j=0}^{p}\left\{z_{j}=0\right\}$, which has codimension larger than two, and the stabilizer of any semi-stable point is $\{\sqrt[p+1]{1}\}$, so that the semi-stable locus coincides with the properly stable one. The corresponding geometric quotient of $\mathbb{P}^{n+p}$ is $\mathbb{P}^{n_{0}} \times \mathbb{P}^{n_{1}} \times \cdots \times \mathbb{P}^{n_{p}}$, and the quotient map is

$$
\begin{gathered}
\phi:\left(\mathbb{P}^{n+p}\right)^{\mathrm{s}} \longrightarrow \mathbb{P}^{n_{0}} \times \mathbb{P}^{n_{1}} \times \ldots \times \mathbb{P}^{n_{p}}, \\
\phi\left(\left[z_{0}, z_{1}, \ldots, z_{p}\right]\right)=\left[z_{0}\right] \times\left[z_{1}\right] \times \ldots \times\left[z_{p}\right] .
\end{gathered}
$$

Any stable map $u: \mathbb{P}^{1} \rightarrow\left(\mathbb{P}^{n+p}\right)^{\mathrm{s}}$ of degree $d$ induces a stable map of multi-degree $(d, d, \ldots, d)$ into the quotient. A rational slice to $\phi$ is just a general $n$-plane, so that the class $\zeta$ appearing in theorem 4.2 is just $\zeta=H^{p}$, where $H$ is the standard generator of the cohomology ring of a projective space.

Consider the simplest case when $n_{0}=n_{1}=1$. Say that we look at rational curves of degree $d$ in $\mathbb{P}^{3}$ and a fortiori of bidegree $(d, d)$ in the quotient, which is $\mathbb{P}^{1} \times \mathbb{P}^{1}$. The class $\zeta=H$ in this case, and according to the result,

$$
G W_{\mathbb{P}^{3}, d}^{4 d-1}(\text { point } \times \underbrace{\text { line } \times \ldots \times \text { line }}_{4 d-2 \text { times }})=G W_{\mathbb{P}^{1} \times \mathbb{P}^{1},(d, d)}^{4 d-1}(\underbrace{\text { point } \times \ldots \times \text { point }}_{4 d-1 \text { times }}) .
$$

In more down-to-earth terms, the number of rational curves of bidegree $(d, d)$ in $\mathbb{P}^{1} \times \mathbb{P}^{1}$ passing through $4 d-1$ general points equals the number of rational curves of degree $d$ in $\mathbb{P}^{3}$ passing through a point and another $4 d-2$ general lines.

4.1.2. Given two integers $m>n>0$, consider the linearized $S l_{n}(\mathbb{C})$-action on $\mathbb{P}^{m n-1}:=\mathbb{P}\left(\operatorname{Hom}\left(\mathbb{C}^{m}, \mathbb{C}^{n}\right)\right)$ given by

$$
S l_{n}(\mathbb{C}) \times \operatorname{Hom}\left(\mathbb{C}^{m}, \mathbb{C}^{n}\right) \longrightarrow \operatorname{Hom}\left(\mathbb{C}^{m}, \mathbb{C}^{n}\right),(g, A) \mapsto g A
$$

The $S l_{n}(\mathbb{C})$-semi-stable points of for this action is the set $\mathbb{P}\left(\operatorname{Hom}\left(\mathbb{C}^{m}, \mathbb{C}^{n}\right)\right)^{\mathrm{ss}}$ of homomorphisms whose rank is $n$. The stabilizer of the stable points is $\mathbb{Z} / n \mathbb{Z}$ but this does not represent any problem because $P S l_{n}(\mathbb{C})$ acts freely, its action linearizes in $\mathcal{O}(n)$ and the corresponding semi-stable locus is the same as that of the $S l_{n}(\mathbb{C})$-action. The quotient is the Grassmannian $G r_{m-n}\left(\mathbb{C}^{m}\right)$ with quotient map

$$
\begin{gathered}
\phi:\left(\mathbb{P}^{m n-1}\right)^{\mathrm{s}} \longrightarrow G r_{m-n}\left(\mathbb{C}^{m}\right), \\
\phi([A])=\operatorname{Ker} A, \quad \forall A \in \mathbb{P}\left(\operatorname{Hom}\left(\mathbb{C}^{m}, \mathbb{C}^{n}\right)\right)^{\mathrm{s}} .
\end{gathered}
$$


There are morphisms $\mathbb{P}^{1} \rightarrow\left(\mathbb{P}^{m n-1}\right)^{\mathrm{ss}}$ in each degree $d>0$, an example being

$$
\left[\zeta_{0}: \zeta_{1}\right] \longmapsto\left(\begin{array}{cccccc}
\zeta_{0}^{d} & \zeta_{1}^{d} & \ldots & 0 & 0 & 0 \\
0 & \ddots & \ddots & \vdots & \vdots & \vdots \\
\vdots & \ddots & \ddots & \zeta_{1}^{d} & 0 & 0 \\
0 & \ldots & 0 & \zeta_{0}^{d} & \zeta_{1}^{d} & 0
\end{array}\right)
$$

The class induced in the Grassmannian is $n d$ times the class of a line. The closure of the inverse image of a point in $G r_{m-n}\left(\mathbb{C}^{m}\right)$ is a $\left(n^{2}-1\right)$-plane in $\mathbb{P}^{m n-1}$; this can be seen easily looking at the inverse image of $\left\langle e_{n+1}, \ldots, e_{m}\right\rangle$, where $e_{1}, \ldots, e_{m}$ is the standard basis of $\mathbb{C}^{m}$. Consequently the rational slice $\zeta$ for the quotient map is just a $n(m-n)$-plane in the projective space, whose class is $H^{n^{2}-1}$.

The pull-back of a Schubert cycle $\sigma_{\lambda}$ in the Grassmannian is $\phi^{*} \sigma_{\lambda}=d(\lambda) H^{|\lambda|} \in$ $A^{|\lambda|}\left(\mathbb{P}^{m n-1}\right)$, for some integer $d(\lambda)$. If $k=\sum_{j=1}^{k}\left|\lambda_{j}\right|-m n d-n(m-n)+3$, we obtain the equality

$$
\begin{aligned}
& G W_{G r_{m-n}\left(\mathbb{C}^{m}\right), n d}^{k}\left(\sigma_{\lambda_{1}} \otimes \ldots \otimes \sigma_{\lambda_{k}}\right) \\
& =\prod_{j=1}^{k} d\left(\lambda_{j}\right) \cdot G W_{\mathbb{P}^{m n-1}, d}^{k}\left(H^{\left|\lambda_{1}\right|+n^{2}-1} \otimes \ldots \otimes H^{\left|\lambda_{k}\right|}\right) .
\end{aligned}
$$

The question which comes to mind is how can be computed the numbers $d(\lambda)$ ? If $\{0\} \subset F_{1} \subset \cdots \subset F_{m}=\mathbb{C}^{m}$ is the standard flag of $\mathbb{C}^{m}$, then

$$
\phi^{*} \sigma_{\lambda}=\left\{[A] \mid \operatorname{dim}\left(\operatorname{Ker} A \cap F_{n+j-\lambda_{j}}\right) \geq j, j=1, \ldots, m-n\right\}
$$

and this is just a degeneration locus of the evaluation homomorphism

$$
\varepsilon: \mathbb{C}^{m} \otimes \mathcal{O}_{\mathbb{P}^{m n-1}}(-1) \longrightarrow \mathbb{C}^{n}
$$

The degree of this subvariety of $\mathbb{P}^{m n-1}$ is given by a determinantal formula which can be found in [8], theorem 14.3 page 249. In the particular case when $\sigma_{\lambda}=\sigma_{k}$, $k=1, \ldots, n$, is a special Schubert cycle, we need to compute the degree $d_{k}$ of the subvariety $\left\{[A] \mid \operatorname{dim}\left(\operatorname{Ker} A \cap F_{n-k+1}\right) \geq 1\right\}=\left\{[A] \mid \varepsilon_{\mid F_{n-k+1}}([A])\right.$ is not injective $\}$ $\subset \mathbb{P}^{m n-1}$. According to [8], theorem 14.4 page $254, d_{k}$ is the coefficient of $H^{k}$ in the development of $1 /(1-H)^{n-k+1}$, which is $\left(\begin{array}{l}n \\ k\end{array}\right)$.

This example can be generalized: take $m>m_{p}>\ldots>m_{1}>0$ integers and let

$$
\begin{gathered}
G:=S l_{m_{p}}(\mathbb{C}) \times \ldots \times S l_{m_{1}}(\mathbb{C}), \\
W:=\operatorname{Hom}\left(\mathbb{C}^{m}, \mathbb{C}^{m_{p}}\right) \oplus \operatorname{Hom}\left(\mathbb{C}^{m_{p}}, \mathbb{C}^{m_{p-1}}\right) \oplus \ldots \oplus \operatorname{Hom}\left(\mathbb{C}^{m_{2}}, \mathbb{C}^{m_{1}}\right) .
\end{gathered}
$$

We consider the linearized $G$-action on $\mathbb{P}(W)$ given by

$$
G \times W \longrightarrow W
$$

$$
\left(g_{p}, \ldots, g_{1}\right) \times\left(A_{p}, \ldots, A_{1}\right):=\left(g_{p} A_{p}, g_{p-1} A_{p-1} \cdot A_{p}, \ldots, g_{1} A_{1} \cdot \ldots \cdot A_{p}\right) .
$$

The $G$-semi-stable locus of $\mathbb{P}(W)$ is the set of $p$-tuples $\left(A_{p}, \ldots, A_{1}\right)$ with all the $A_{j}$ 's surjective, and the stabilizer of these points is $\mathbb{Z} / m_{p} \mathbb{Z} \times \ldots \times \mathbb{Z} / m_{1} \mathbb{Z}$. 
Denoting $d_{j}:=m-m_{j}$, the quotient for the action is

$$
\begin{aligned}
\phi: \mathbb{P}(W)^{\mathrm{s}} \longrightarrow \mathbb{F}_{d_{p}, \ldots, d_{1}}(W), \\
\left(A_{p}, \ldots, A_{1}\right) \longmapsto\left(\operatorname{Ker} A_{p}, \ldots, \operatorname{Ker}\left(A_{1} \cdot \ldots \cdot A_{p}\right)\right),
\end{aligned}
$$

where $\mathbb{F}_{d_{p}, \ldots, d_{1}}(W):=\left\{0 \subset W_{d_{p}} \subset \ldots \subset W_{d_{1}} \subset W\right\}$ is the variety of flags of dimensions $d_{p}<\ldots<d_{1}$ in $W$.

\section{Second application: Hamiltonian GW-invariants}

The second application concerns the so-called Hamiltonian Gromov-Witten invariants which were recently introduced in $[6,14]$. The purpose of this and the next sections is to put into an algebro-geometric perspective the construction performed in these preprints and to show how is that related to the problem studied in this article, at least in the case of torus actions.

In what follows, $K$ will denote the compact torus $\left(S^{1}\right)^{r}$ and $G=K^{c}=\left(\mathbb{C}^{*}\right)^{r}$ will be its complexification. We assume that $G$ acts holomorphically on a projective variety $X$ and that the action is linearized in a very ample line bundle $\mathcal{O}_{X}(1) \rightarrow X$. Then the maximal compact subgroup $K$ will preserve a symplectic form on $X$ representing the first Chern class of $\mathcal{O}_{X}(1)$, and we get a Hamiltonian action on $X$. The explicit formula for the corresponding moment map can be found in [10], lemma 2.5 page 24 .

In all the rest, $C$ denotes a smooth projective curve with a Kähler metric on it. As usual, EG $\rightarrow \mathrm{BG}$ and $\mathrm{EK} \rightarrow \mathrm{BK}$ will stand for the universal $G$ and $K$ bundles; they are uniquely determined (up to homotopy) by the condition that are contractible and $G$ and $K$ act freely on them, so that we may take $\mathrm{EG}=\mathrm{EK}=: E$.

The $K$-equivariant homology of $X$ is defined as $H_{*}^{K}(X):=H_{*}\left(E \times_{K} X\right)$ and elements of it can be constructed as follows: one starts with a principal $K$-bundle $P \rightarrow M$ over a closed $\mathscr{C}^{\infty}$-manifold $M$ of real dimension $d$ together with a $K$ equivariant map $U: P \rightarrow X$. The $d$-dimensional equivariant homology class defined by this data is the image of the fundamental class of $M$ under

$$
H_{d}(M) \cong H_{d}^{K}(P) \longrightarrow H_{d}^{K}(X) \text {. }
$$

For every $K$-equivariant 2-homology class $B \in H_{2}^{K}(X ; \mathbb{Z})$ there is a closed Riemann surface $\Sigma$ and a principal $K$-bundle $P \rightarrow \Sigma$ together with a $K$-equivariant map $U: P \rightarrow X$ representing the class $B$. Moreover, if $\Sigma$ is connected and $P, P^{\prime} \rightarrow \Sigma$ represent the same class, then $P$ and $P^{\prime}$ are isomorphic as $K$-bundles. In other words, the choice of an equivariant homology class uniquely determines the topological type of the principal bundles, over a fixed Riemann surface, which can represent this homology class.

Given a principal $K$-bundle $P \rightarrow C$, the complexified bundle $P \times_{K} G$ will be denoted $P^{c}$. The gauge groups of $P$ and $P^{c}$ are respectively

$$
\mathscr{G}(P)=\left\{f: C \rightarrow\left(S^{1}\right)^{r}\right\} \quad \text { and } \quad \mathscr{G}^{c}(P)=\left\{f: C \rightarrow\left(\mathbb{C}^{*}\right)^{r}\right\} .
$$


A base point $\zeta_{0} \in C$ will be fixed once for all. Corresponding to it we will consider the based gauges $\mathscr{G}_{0}^{c}(P)$ of $P^{c}$ which are the identity at $\zeta_{0}$. The full gauge group is then the direct product of $\left(\mathbb{C}^{*}\right)^{r}$ with the based gauge group.

\subsection{A-holomorphic maps}

Now we turn to another ingredient used in the definition of the Hamiltonian GWinvariants.

Definition 5.1.1. (i) Given a connection $A \in \mathscr{A}(P)$ and a $K$-equivariant map $U: P \rightarrow X$, the operator $\mathrm{d}_{A} U$ is defined as

$$
T_{p} P \ni w \longmapsto \mathrm{d} U_{p}(w)+\xi(A(w))_{U(p)},
$$

where $\xi(a)_{x}$ is the tangent vector at $x \in X$ determined by $a \in \operatorname{Lie}(K)$.

(ii) A $K$-equivariant map $U:(P, A) \rightarrow X$ is called $A$-holomorphic if $\bar{\partial}_{A} U=0$, where

$$
\bar{\partial}_{A} U:=\frac{1}{2}\left(\mathrm{~d}_{A} U+J_{X} \circ \mathrm{d}_{A} U \circ J_{C}\right) .
$$

The notation $J_{C}$ stands for the complex structure induced on the $A$-horizontal spaces of $P$ by the complex structure of $C$.

In more down to earth terms, a $K$-equivariant map $U:(P, A) \rightarrow X$ is $A$ holomorphic if and only if

$$
\mathrm{d} U_{p}(\widetilde{J v})=J \mathrm{~d} U_{p}(\widetilde{v})
$$

where $\widetilde{v}$ and $\widetilde{J v}$ denote respectively the $A$-horizontal liftings in $p \in P$ of the vectors $v, J v \in T C$.

Definition 5.1.2. (Cf. [6] section 3.2.) Denote

$$
\mathfrak{X}_{B}:=\left\{(U, A) \in C_{K}^{\infty}(P, X ; B) \times \mathscr{A}(P) \mid \bar{\partial}_{A} U=0\right\}
$$

the space of $K$-equivariant, $A$-holomorphic smooth maps which represent the class $B \in H_{2}^{K}(X)$.

Any $K$-equivariant map $U: P \rightarrow X$ induces a map $\bar{u}: C \rightarrow P \times_{K} X$. Then $U$ is $A$-holomorphic if and only if $\bar{u}$ is holomorphic. One has to be careful with the (integrable) complex structure on $P \times_{K} X$ which is induced by the connection $A$. For vectors tangent to the fibres of $P \times_{K} X \rightarrow C$ the complex structure agrees with that of $X$, while for $v \in T C$ (in a local trivialization $P \cong C \times K$ ),

$$
J_{P \times{ }_{K} X}(v)=J v+\xi(A(J v))-J \xi(A(v)) .
$$

In this formula and in all the rest of the paper, for $a \in \operatorname{Lie}(K), \xi(a)$ will denote the vector field on $X$ induced by the infinitesimal $K$-action.

Clearly, the real gauges act on $\mathfrak{X}_{B}$ but it turns out that the complex gauges act also. The formula for it is given in the lemma below. 
Lemma 5.1.3. The complex gauges $\mathscr{G}^{c}(P)$ act on $\mathfrak{X}_{B}$ as $f \times(U, A) \longmapsto(f \cdot U, f \cdot A)$, where

$$
(f \cdot U)(p):=f^{-1}(p) U(p)
$$

and

$$
f \cdot A:=A+\left(f^{-1} \mathrm{~d} f\right)_{\mathfrak{k}}+*\left(f^{-1} \mathrm{~d} f\right)_{i \mathfrak{k}} .
$$

Some explanation is in order: any $a \in \operatorname{Lie}(G)=\operatorname{Lie}(K) \oplus i \operatorname{Lie}(K)$ can be uniquely written $a=a_{\mathfrak{k}}+i a_{\mathfrak{i k}}$, with $a_{\mathfrak{k}}, a_{\mathfrak{k}} \in \operatorname{Lie}(K)$. The $*$ in the formula represents the Hodge star operator on $C$.

Proof. It is clear that formula (5.2) just extends the action of the real gauges by composition on the right. We shall prove the formula for the action of $f$ on $A$ by finding a connection $A^{\prime}$ on $P$ which makes $A^{\prime}$-holomorphic the map $U^{\prime}:=f \cdot U$. For doing computations we use a local trivialization of $P$, so that $P$ itself may be assumed trivial (as long as the objects found in the end are globally defined).

In what follows, $\zeta$ denotes a point on $C$. By assumption $P=C \times K$ and $U\left(\zeta, g^{-1}\right)=g u(\zeta)$, for some $u: C \rightarrow X$. I want to find a connection $A^{\prime}$ on $P$ such that $\bar{\partial}_{A^{\prime}} U^{\prime}=0$. Since $U^{\prime}$ is $K$-equivariant, it is enough to check this condition at points $(\zeta, 1) \in P$. Because $U$ is $A$-holomorphic,

$$
\mathrm{d} U_{(\zeta, 1)}(J v-A(J v))=J \mathrm{~d} U_{(\zeta, 1)}(v-A(v))
$$

for $v \in T_{\zeta} C$, or equivalently

$$
\mathrm{d} u_{\zeta}(J v)+\xi(A(J v))_{u(\zeta)}=J \mathrm{~d} u_{\zeta}(v)+J \xi(A(v))_{u(\zeta)} .
$$

Formula (5.2) implies that

$$
\begin{aligned}
\mathrm{d} U_{(\zeta, 1)}^{\prime}\left(J v-A^{\prime}(J v)\right) & =f^{-1}(\zeta) \mathrm{d} u_{\zeta}(J v)-f^{-1}(\zeta) \xi\left(\left(f^{-1} \mathrm{~d} f\right)(J v)\right)_{u(\zeta)} \\
& +f^{-1}(\zeta) \xi\left(A^{\prime}(J v)\right)_{u(\zeta)}
\end{aligned}
$$

and

$$
\begin{aligned}
J \mathrm{~d} U_{(\zeta, 1)}^{\prime}\left(v-A^{\prime}(v)\right) & =f^{-1}(\zeta) J \mathrm{~d} u_{\zeta}(v)-f^{-1}(\zeta) J \xi\left(\left(f^{-1} \mathrm{~d} f\right)(v)\right)_{u(\zeta)} \\
& +f^{-1}(\zeta) J \xi\left(A^{\prime}(v)\right)_{u(\zeta)}
\end{aligned}
$$

For $U^{\prime}$ to be $A^{\prime}$-holomorphic it is necessary and sufficient that the difference of these two quantities is zero. Imposing this condition, we find

$$
\begin{aligned}
0 & =J \mathrm{~d} u_{\zeta}(v)-\mathrm{d} u_{\zeta}(J v)-J \xi\left(\left(f^{-1} \mathrm{~d} f\right)(v)\right)_{u(\zeta)}+\xi\left(\left(f^{-1} \mathrm{~d} f\right)(J v)\right)_{u(\zeta)} \\
& +J \xi\left(A^{\prime}(v)\right)_{u(\zeta)}-\xi\left(A^{\prime}(J v)\right)_{u(\zeta)} \\
& \stackrel{(5.4)}{=} \xi(A(J v))_{u(\zeta)}-J \xi(A(v))_{u(\zeta)}-\xi\left(A^{\prime}(J v)\right)_{u(\zeta)}+J \xi\left(A^{\prime}(v)\right)_{u(\zeta)} \\
& +\xi\left(\left(f^{-1} \mathrm{~d} f\right)(J v)\right)_{u(\zeta)}-J \xi\left(\left(f^{-1} \mathrm{~d} f\right)(v)\right)_{u(\zeta)} \\
& =\xi\left(A(J v)-A^{\prime}(J v)\right)_{u(\zeta)}-J \xi\left(A(v)-A^{\prime}(v)\right)_{u(\zeta)} \\
& +\xi\left(\left(f^{-1} \mathrm{~d} f\right)(J v)\right)_{u(\zeta)}-J \xi\left(\left(f^{-1} \mathrm{~d} f\right)(v)\right)_{u(\zeta)}
\end{aligned}
$$


It remains to separate the $\operatorname{Lie}(K)$ and $i \operatorname{Lie}(K)$ components of the last line.

$$
\begin{aligned}
& \xi\left(\left(f^{-1} \mathrm{~d} f\right)(J v)\right)-J \xi\left(\left(f^{-1} \mathrm{~d} f\right)(v)\right) \\
& =\xi\left(\left(f^{-1} \mathrm{~d} f\right)_{\mathfrak{k}}(J v)+i\left(f^{-1} \mathrm{~d} f\right)_{i \mathfrak{k}}(J v)\right)-\xi\left(i\left(f^{-1} \mathrm{~d} f\right)_{\mathfrak{k}}(v)-\left(f^{-1} \mathrm{~d} f\right)_{i \mathfrak{k}}(v)\right) \\
& =\xi\left(\left(f^{-1} \mathrm{~d} f\right)_{\mathfrak{k}}(J v)+\left(f^{-1} \mathrm{~d} f\right)_{i \mathfrak{k}}(v)\right)-J \xi\left(\left(f^{-1} \mathrm{~d} f\right)_{\mathfrak{k}}(v)-\left(f^{-1} \mathrm{~d} f\right)_{i \mathfrak{k}}(J v)\right) .
\end{aligned}
$$

Inserting this into the previous relation, we obtain

$$
\begin{aligned}
0 & =\xi\left(A(J v)-A^{\prime}(J v)+\left(f^{-1} \mathrm{~d} f\right)_{\mathfrak{k}}(J v)+\left(f^{-1} \mathrm{~d} f\right)_{i \mathfrak{k}}(v)\right) \\
& -J \xi\left(A(v)-A^{\prime}(v)+\left(f^{-1} \mathrm{~d} f\right)_{\mathfrak{k}}(v)-\left(f^{-1} \mathrm{~d} f\right)_{i \mathfrak{k}}(J v)\right) .
\end{aligned}
$$

For $A^{\prime}$ defined by

$$
A^{\prime}=A+\left(f^{-1} \mathrm{~d} f\right)_{\mathfrak{k}}-\left(f^{-1} \mathrm{~d} f\right)_{i \mathfrak{k}} \circ J
$$

the last equality is satisfied. Notice that in general this is the only possible choice for $A^{\prime}$ since the vectors $\xi$ and $J \xi$ are linearly independent in most cases.

Using local normal coordinates on $C$, it follows that for any 1-form $\alpha \in \Omega_{C}^{1}$, $\alpha \circ J=-(* \alpha)$.

Remark 5.1.4. (i) It follows from formula (5.3) that the $\mathscr{G}_{0}^{c}(P)$-action on $\mathfrak{X}_{B}$ is free.

(ii) Any $f: C \rightarrow\left(\mathbb{C}^{*}\right)^{r}$ is of the form $f(\zeta)=R(\zeta) \varphi(\zeta)$, with $R: C \rightarrow \mathbb{R}^{r}$ and $\varphi: C \rightarrow\left(S^{1}\right)^{r}$. Formula (5.3) becomes

$$
f \cdot A=A+\varphi^{-1} \mathrm{~d} \varphi-i * \mathrm{~d}(\log R) .
$$

The form $\varphi^{-1} \mathrm{~d} \varphi$ is closed, but not necessarily exact; it is exact if and only if $\varphi_{*}: \pi_{1}(C) \rightarrow\left(\pi_{1}\left(S^{1}\right)\right)^{r}$ is the zero homomorphism, or equivalently $\varphi=\exp (i \theta)$ for some $\theta: C \rightarrow \mathbb{R}^{r}$. However it always defines an integral 1-cohomology class and conversely, any integral 1-cohomology class can be represented in this form.

Using the Hodge decomposition of $\Omega_{C}^{1}$, this discussion implies that the pointed complex gauge equivalence classes of connections in $P$ are parameterized by

$$
\mathbb{H}^{1}\left(C, \mathbb{R}^{r}\right) / \mathbb{H}^{1}\left(C, \mathbb{Z}^{r}\right)
$$

where $\mathbb{H}^{1}\left(C, \mathbb{R}^{r}\right)$ denotes the space of harmonic $\mathbb{R}^{r}$-valued 1-forms on $C$. This quotient is just the $r^{\text {th }}$ power of the familiar Picard variety of $C$, when $\mathbb{H}^{1}\left(C, \mathbb{R}^{r}\right)$ is given the complex structure defined by the Hodge-star of $C$.

(iii) In the genus zero case, i.e. $C \cong \mathbb{P}^{1}$, all gauges admit a globally defined logarithm. Therefore all connections are gauge equivalent, which is the same saying that in a given topological principal bundle $P \rightarrow \mathbb{P}^{1}$ there is only one equivalence class of holomorphic structures. 


\subsection{Short digression on the Picard variety}

All the statements in this section should be well known, but we are recalling them in order to fix the notations. As I have already mentioned, the quotient

$$
\mathbb{H}^{1}\left(C, \mathbb{R}^{r}\right) / \mathbb{H}^{1}\left(C, \mathbb{Z}^{r}\right)=\left(\mathbb{H}^{1}(C, \mathbb{R}) / \mathbb{H}^{1}(C, \mathbb{Z})\right)^{r}=\left(P i c^{0} C\right)^{r}
$$

is the $r^{\text {th }}$ power of the Picard variety of $C$, when $\mathbb{H}^{1}\left(C, \mathbb{R}^{r}\right)$ is regarded as a complex vector space with complex structure given by the Hodge-star of $C$. It is a projective torus which parameterizes topologically trivial, holomorphic principal $\left(\mathbb{C}^{*}\right)^{r}$-bundles over $C$. We are somewhat sloppy at this point, because the $r$ copies of the $\mathbb{C}^{*}$ 's are labeled in our case, so we can talk about the first copy, the second one, and so forth. Otherwise, the $\left(\mathbb{C}^{*}\right)^{r}$-bundles over $C$ would be parameterized by $\operatorname{Sym}^{r}\left(\left(P i c^{0} C\right)^{r}\right):=\left(P i c^{0} C\right)^{r} / \mathfrak{S}_{r}$.

Let

$$
\mathcal{P}_{r}^{c} \longrightarrow\left(P i c^{0} C\right)^{r} \times C
$$

be a universal principal $\left(\mathbb{C}^{*}\right)^{r}$-bundle. It has the property that for any point $\tau \in\left(P i c^{0} C\right)^{r}$, the restriction $\left(\mathcal{P}_{r}^{c}\right)_{\tau} \longrightarrow C$ represents the point $\tau$. We shall be interested in describing a connection in this bundle which induces its holomorphic structure.

Let us start with the

Lemma 5.2.1. For any $\alpha \in \mathbb{H}^{1}\left(C ; \mathbb{Z}^{r}\right)$, there is a unique $\varphi_{\alpha}: C \rightarrow\left(S^{1}\right)^{r}$ such that $\varphi_{\alpha}\left(\zeta_{0}\right)=1$ and $\varphi_{\alpha}^{-1} \mathrm{~d} \varphi_{\alpha}=\alpha$. (The point $\zeta_{0} \in C$ was fixed from the beginning).

Proof. Clearly, we may assume that $r=1$. The uniqueness part is immediate. For the existence part, notice that if $\alpha$ is exact, i.e. $\alpha=\mathrm{d} \theta$ for $\theta: C \rightarrow \mathbb{R}$, then $\varphi:=\exp \left(i\left(\theta-\theta\left(\zeta_{0}\right)\right)\right)$ does the job. Homotopy classes of maps $C \rightarrow \mathbb{R}$ are parameterized by $\operatorname{Hom}_{\mathbb{Z}}\left(H^{1}(C ; \mathbb{Z}) ; \mathbb{Z}\right) \cong H^{1}(C ; \mathbb{Z})$, so for $\alpha \in \mathbb{H}^{1}(C ; \mathbb{Z})$ there exists $\varphi^{\prime}: C \rightarrow S^{1} \operatorname{such} \varphi^{\prime}\left(\zeta_{0}\right)=1$ and $\left[\left(\varphi^{\prime}\right)^{-1} \mathrm{~d} \varphi^{\prime}\right]=[\alpha] \in H^{1}(C ; \mathbb{Z})$. By the discussion above, there exists $\varphi_{0}: C \rightarrow S^{1}$ such that $\varphi_{0}\left(\zeta_{0}\right)=1$ and $\varphi_{0}^{-1} \mathrm{~d} \varphi_{0}=\alpha-\left(\varphi^{\prime}\right)^{-1} \mathrm{~d} \varphi^{\prime}$. Now $\varphi:=\varphi_{0} \varphi^{\prime}$ will be convenient.

Remark 5.2.2. The map

$$
\mathbb{H}^{1}\left(C ; \mathbb{Z}^{r}\right) \ni \alpha \longmapsto \varphi_{\alpha} \in \mathscr{C}^{\infty}\left(C,\left(S^{1}\right)^{r}\right)
$$

is a morphism of groups i.e. $\varphi_{\alpha} \varphi_{\beta}=\varphi_{\alpha+\beta}$.

Fix once for all a real connection $A_{0}$ in the smooth $\left(\mathbb{C}^{*}\right)^{r}$-bundle $P^{c} \rightarrow C$ i.e. one coming from a connection in the real $\left(S^{1}\right)^{r}$-bundle.

Lemma 5.2.3. (i) On $\mathbb{H}^{1}\left(C ; \mathbb{R}^{r}\right) \times C$, there is a natural, closed $\mathbb{R}^{r}$-valued 1 -form $\chi$ which is defined by

$$
\chi_{(A, \zeta)}(a, v):=A(v) \quad \text { for } \quad(a, v) \in T_{(A, \zeta)}\left(\mathbb{H}^{1}\left(C ; \mathbb{R}^{r}\right) \times C\right) .
$$


(ii) The (real) connection $\mathcal{A}:=A_{0}+\chi$ defines a holomorphic structure on the bundle $\operatorname{pr}_{C}^{*} P^{c} \rightarrow \mathbb{H}^{1}\left(C ; \mathbb{R}^{r}\right) \times C$.

Proof. The curvature of $\mathcal{A}$ is $F_{\mathcal{A}}=\operatorname{pr}_{C}^{*} F_{A_{0}}+\mathrm{d} \chi$. For $a \in \mathbb{H}^{1}\left(C ; \mathbb{R}^{r}\right)$ and $v \in T C$, $\mathrm{d} \chi((a, 0),(0, v))=a(v)$, and $\mathrm{d} \chi$ evaluates zero on other pairs of vectors.

It is easy to see that for any 1-form $a$ on $C$ and any tangent vector $v$ to $C$, $(* a)(J v)=a(v)$. This implies that the connection $\mathcal{A}$ defines indeed a holomorphic structure in $\operatorname{pr}_{C}^{*} P^{c}$ because its curvature is a $(1,1)$-form on $\mathbb{H}^{1}\left(C ; \mathbb{R}^{r}\right) \times C$.

Proposition 5.2.4. (i) The group $\mathbb{H}^{1}\left(C ; \mathbb{Z}^{r}\right)$ acts holomorphically, by real gauges, on $\operatorname{pr}_{C}^{*} P^{c}$ by

$$
\begin{gathered}
\mathbb{H}^{1}\left(C ; \mathbb{Z}^{r}\right) \times\left(\mathbb{H}^{1}\left(C ; \mathbb{R}^{r}\right) \times P^{c}\right) \longrightarrow \mathbb{H}^{1}\left(C ; \mathbb{R}^{r}\right) \times P^{c} \\
\alpha \times(A, p):=\left(A+\alpha, R_{\varphi_{\alpha}} p\right) .
\end{gathered}
$$

(ii) The holomorphic principal bundle

$$
\mathcal{P}_{r}^{c}\left(A_{0}\right):=\operatorname{pr}_{C}^{*} P^{c} / \mathbb{H}^{1}\left(C ; \mathbb{Z}^{r}\right) \longrightarrow\left(\operatorname{Pic}^{0} C\right)^{r} \times C
$$

is a universal principal $\left(\mathbb{C}^{*}\right)^{r}$-bundle which parameterizes holomorphic bundles over $C$ having fixed topological type defined by $A_{0}$. It also comes with the connection induced by $\mathcal{A}$.

Proof. (i) Remark 5.2.2 implies that the formula above is indeed an action. It is also holomorphic because $\mathbb{H}^{1}\left(C ; \mathbb{Z}^{r}\right)$ preserves the connection $\mathcal{A}$; indeed, $A+\alpha=\varphi_{\alpha} A$.

(ii) The statement is a direct consequence of 5.1.4.

We should say that we have worked with complex principal bundles throughout this section because the accent was put on their holomorphic structure. But $\mathcal{P}_{r}^{c}\left(A_{0}\right)$ is the complexification of a real $\left(S^{1}\right)^{r}$-bundle $\mathcal{P}_{r}\left(A_{0}\right) \rightarrow\left(P i c^{0} C\right)^{r} \times C$ and the connection $\mathcal{A}$ comes from a connection in $\mathcal{P}_{r}\left(A_{0}\right)$, because the connection $A_{0}$, fixed from the beginning, was real and the action on $\operatorname{pr}_{C}^{*} P^{c}$ was done by real gauges.

\section{Moduli spaces}

In this section we shall see that the spaces introduced in [6] and [14] for defining invariants of Hamiltonian group actions have nice algebraic interpretations.

The authors of [6] introduce (a perturbation of)

$$
\tilde{S}_{C, k}(X ; B):=\left\{(U, A) \in \mathfrak{X}_{B} \mid * F_{A}+m \circ U=0\right\} \times\left(P^{k}\right)_{o} / \mathscr{G}(P)
$$

and

$$
S_{C, k}(X ; B):=\tilde{S}_{C, k}(X ; B) / G^{k} .
$$


In the definition above $m: X \rightarrow \mathbb{R}^{r}$ is the moment map corresponding to the $\left(S^{1}\right)^{r}$-action and $\left(P^{k}\right)_{o}$ is the open set in $P^{k}$ consisting of $k$ points which project to $k$ distinct points of $C$. The group actions are as follows:

$$
f \times\left((U, A) \times\left(p_{1}, \ldots, p_{k}\right)\right):=(f \cdot U, f \cdot A) \times\left(R_{f\left(p_{1}\right)} p_{1}, \ldots, R_{f\left(p_{k}\right)} p_{k}\right)
$$

and

$$
\left(g_{1}, \ldots, g_{k}\right) \times\left[(U, A) \times\left(p_{1}, \ldots, p_{k}\right)\right]:=\left[(U, A) \times\left(R_{g_{1}} p_{1}, \ldots, R_{g_{k}} p_{k}\right)\right],
$$

for $f \in \mathscr{G}(P)$ and $g_{1}, \ldots, g_{k} \in K=\left(S^{1}\right)^{r}$. The letter ' $R$ ' denotes the right action of $G$ on the principal bundle. The expected dimension of this space is

$$
2 D:=\exp \operatorname{dim}_{\mathbb{R}} S_{C, k}(X ; B)=2(1-g)(n-r)+2 c_{1}^{K}(X) \cdot B+2 k,
$$

where $c_{1}^{K}(X)$ denote the $K$-equivariant first Chern class of $X$.

The space $S_{C, k}(X, A)$ can also be expressed as the infinite dimensional invariant quotient as

$$
S_{C, k}(X, B)=\left(\mathfrak{X}_{B}^{\mathrm{s}} \times\left(P^{c}\right)_{o}^{k} / \mathscr{G}^{c}(P)\right) / G^{k} .
$$

The notation $\mathfrak{X}_{B}^{\mathrm{s}} \subset \mathfrak{X}_{B}$ stands for the set of so-called stable pairs and $\mathscr{G}^{c}(P)$ acts on $\mathfrak{X}_{B}$ as described in lemma 5.1.3.

For the present purposes, it will be more convenient to use this second description. Since $\mathscr{G}^{c}(P)=\mathscr{G}_{0}^{c}(P) \times G$ and the actions of $\mathscr{G}^{c}(P)$ and $G^{k}$ on $\mathfrak{X}_{B}$ commute, $S_{C, k}(X ; B)$ can be constructed in a different way: first take the quotient for the free $\mathscr{G}_{0}^{c}(P) \times G^{k}$-action (which is finite dimensional) and after make the invariant quotient for the remaining $G$-action.

$K$-equivariant maps $U: P \rightarrow X$ and $G$-equivariant maps $U^{c}: P^{c} \rightarrow X$ will be used interchangeably. That there is no harm in doing so follows from the fact that any $K$-equivariant $U$ defines (in the obvious way) a $G$-equivariant $U^{c}$; conversely, any such $U^{c}$ defines a corresponding $U$ composing it with the inclusion $j: P \hookrightarrow P^{c}$.

Lemma 6.1. (i) The variety $\bar{X}$ defined by

$$
\bar{X}:=\mathcal{P}_{r}^{c}\left(A_{0}\right) \times_{G} X=\mathcal{P}_{r}\left(A_{0}\right) \times_{K} X,
$$

carries a natural structure of a complex projective variety. Its complex dimension is $\operatorname{dim} \bar{X}=g r+\operatorname{dim} X+1$, where $g$ is the genus of $C$ and $r=\operatorname{dim} G$.

(ii) Any K-equivariant, A-holomorphic map $U: P \rightarrow X$, with $A \in \mathscr{A}(P)$, which represents an equivariant 2 -homology class $B \in H_{2}^{K}(X ; \mathbb{Z})$ defines a holomorphic map $\bar{u}: C \rightarrow \bar{X}$ which represents a class $\bar{B} \in H_{2}(\bar{X} ; \mathbb{Z})$ depending on $B$ only. If $\pi: \bar{X} \rightarrow\left(\mathrm{Pic}^{0} C\right)^{r} \times C$ is the natural projection, $\pi_{*} \bar{B}=0 \oplus[C]$. Moreover, $c_{1}^{K}(X) \cdot B=c_{1}\left(T_{\bar{X}}^{\mathrm{rel}}\right) \cdot \bar{B}$, where $T_{\bar{X}}^{\mathrm{rel}}$ denotes the $\pi$-relative tangent bundle.

(iii) Consider an A-holomorphic, K-equivariant map $U: P \rightarrow X$ and $g \in$ $\mathscr{G}_{0}^{c}(P)$. Then $U$ and $g U$ define the same map $C \rightarrow \bar{X}$. 
Proof. (i) $\bar{X}$ has a holomorphic structure because $\mathcal{P}_{r}^{c}\left(A_{0}\right) \rightarrow\left(\operatorname{Pic}^{0} C\right)^{r} \times C$ is a holomorphic bundle, according to lemma 5.2.3. That it is also a projective variety follows from the fact that the Picard torus is projective.

(ii) Remark 5.1.4 implies that given $U:(P, A) \rightarrow X$ there is a unique $f_{A} \in$ $\mathscr{G}_{0}^{c}(P)$ (depending on $A$ ) such that

$$
f_{A} \cdot A=\text { harmonic part of } A-A_{0}=: h\left(A-A_{0}\right) \in \mathbb{H}^{1}\left(C ; \mathbb{R}^{r}\right) .
$$

The composed map

$$
P \stackrel{\left(\left\{h\left(A-A_{0}\right)\right\} \times \operatorname{id}_{P}\right) \times f_{A} U}{\longrightarrow} \underbrace{\mathbb{H}^{1}\left(C ; \mathbb{R}^{r}\right) \times P}_{=\text {pr }_{C}^{*} P} \times X \longrightarrow \mathcal{P}_{r}\left(A_{0}\right) \times X
$$

is $K$-equivariant and therefore defines

$$
\bar{u}: C \longrightarrow \mathcal{P}_{r}\left(A_{0}\right) \times_{K} X=\bar{X} .
$$

Since $f_{A} U: P \rightarrow X$ is $f_{A} A$-holomorphic, it follows that this map is holomorphic. If $p_{\zeta} \in P$ (or in $P^{c}$ ) denotes a point lying over $\zeta \in C$, the explicit formula for $\bar{u}$ is

$$
\zeta \stackrel{\bar{u}}{\longmapsto}\left[\left[h\left(A-A_{0}\right), p_{\zeta}\right],\left(f_{A} U\right)\left(p_{\zeta}\right)\right],
$$

where the square brackets denote obvious equivalence classes.

Suppose that $P=\rho^{*} E$ for a map $\rho: C \rightarrow$ BK. Then from the commutative diagram

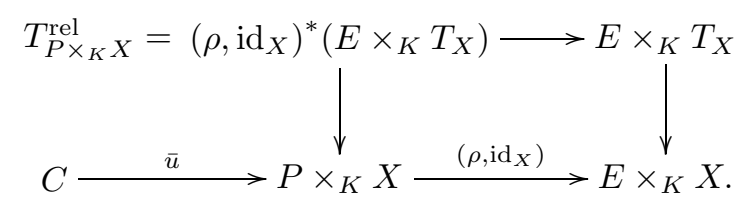

we can see that the homology class $\bar{B}$ depends only on the $K$-equivariant class $B$ and also that $c_{1}^{K}(X) \cdot B=c_{1}\left(T_{\bar{X}}^{\mathrm{rel}}\right) \cdot \bar{B}$.

(iii) Consider now $U: P \rightarrow X$ and $g \in \mathscr{G}_{0}^{c}(P), g=R \cdot \varphi$ with $\varphi: C \rightarrow\left(S^{1}\right)^{r}$ and $R: C \rightarrow \mathbb{R}^{r}$. According to (5.5), $g A=A+\varphi^{-1} \mathrm{~d} \varphi-i * \mathrm{~d}(\log R)$, so

$$
h\left(g A-A_{0}\right)=h\left(A-A_{0}\right)+h\left(\varphi^{-1} \mathrm{~d} \varphi\right) .
$$

Notice that $\alpha_{\varphi}:=h\left(\varphi^{-1} \mathrm{~d} \varphi\right)$ is actually an integral $\mathbb{R}^{r}$-valued harmonic form and according to lemma 5.2.1 there is a unique $\psi_{\varphi} \in \mathscr{G}_{0}(P)$ such that $\psi_{\varphi}^{-1} \mathrm{~d} \psi_{\varphi}=\alpha_{\varphi}$. I claim that $f_{g A}=\psi_{\varphi} f_{A} g^{-1}$, i.e. that $\left(\psi_{\varphi} f_{A} g^{-1}\right)(g A)=A_{0}+h\left(g A-A_{0}\right)$. Indeed,

$$
\begin{aligned}
\left(\psi_{\varphi} f_{A} g^{-1}\right)(g A) & =\psi_{\varphi}\left(f_{A} A\right)=\psi_{\varphi}\left(A_{0}+h\left(A-A_{0}\right)\right) \\
& =A_{0}+h\left(A-A_{0}\right)+\psi_{\varphi}^{-1} \mathrm{~d} \psi_{\varphi} \\
& =A_{0}+h\left(A-A_{0}\right)+\alpha_{\varphi}=A_{0}+h\left(g A-A_{0}\right) .
\end{aligned}
$$

We are going to check now that $U$ and $g U$ induce the same (holomorphic) map 
$C \rightarrow \bar{X}$. Using formula (6.6), $g U$ reads

$$
\begin{aligned}
\zeta & \longmapsto\left[\left[h\left(A-A_{0}\right)+\alpha_{\varphi}, p_{\zeta}\right], \psi_{\varphi}^{-1}\left(p_{\zeta}\right) \cdot\left(f_{A} U\right)\left(p_{\zeta}\right)\right] \\
& =\left[\left[h\left(A-A_{0}\right)+\alpha_{\varphi}, R_{\psi_{\varphi}} p_{\zeta}\right],\left(f_{A} U\right)\left(p_{\zeta}\right)\right] \\
& =\left[\left[h\left(A-A_{0}\right), p_{\zeta}\right],\left(f_{A} U\right)\left(p_{\zeta}\right)\right] .
\end{aligned}
$$

This finishes the proof of the lemma. (6.2).

The next proposition gives the algebro-geometric interpretation of the space

Proposition 6.2. There is a one-to-one map

$$
\left(\mathfrak{X}_{B} \times\left(P^{c}\right)_{o}^{k}\right) /\left(\mathscr{G}_{0}^{c}(P) \times G^{k}\right) \stackrel{1: 1}{\longleftrightarrow} M_{C, k}(\bar{X} ; \bar{B}),
$$

where $M_{C, k}(\bar{X} ; \bar{B})$ denotes the space of stable maps $(C, \underline{x}) \rightarrow \bar{X}$ with $k$ marked points $\underline{x} \in\left(C^{k}\right)_{o}$ and representing the 2 -homology class $\bar{B}$. As usual, $\left(C^{k}\right)_{o}$ denotes the complement in $C^{k}$ of the diagonals.

Proof. Consider an $A$-holomorphic, $K$-equivariant map $U: P \rightarrow X$ together with $k$ marked points $p \in\left(P^{c}\right)_{o}^{k}$ and let $\underline{x}:=\pi_{C}(p) \in\left(C^{k}\right)_{o}$. Lemma 6.1 says that this data induces a morphism $C \rightarrow \bar{X}$ and moreover, it does not depend on the $\mathscr{G}_{0}^{c}(P)$-orbit of $(U, A)$. So we get a map

$$
F:\left(\mathfrak{X}_{B} \times\left(P^{c}\right)_{o}^{k}\right) / \mathscr{G}_{0}^{c}(P) \longrightarrow M_{C, k}(\bar{X} ; \bar{B}) .
$$

This map is clearly $G^{k}$-invariant and therefore descends to the quotient

$$
F:\left(\mathfrak{X}_{B} \times\left(P^{c}\right)_{o}^{k}\right) /\left(\mathscr{G}_{0}^{c}(P) \times G^{k}\right) \longrightarrow M_{C, k}(\bar{X} ; \bar{B}) .
$$

Because the composition $\pi \bar{u}=\{\tau\} \times \operatorname{id}_{C}$, for some $\tau \in\left(P i c^{0} C\right)^{r}$, the map $\bar{u}$ is in fact a representative for the corresponding stable map (see definition 1.1).

The map $F$ is clearly surjective: given a point $(\bar{u}, \underline{x}) \in M_{C, k}(\bar{X} ; \bar{B})$, consider the diagram

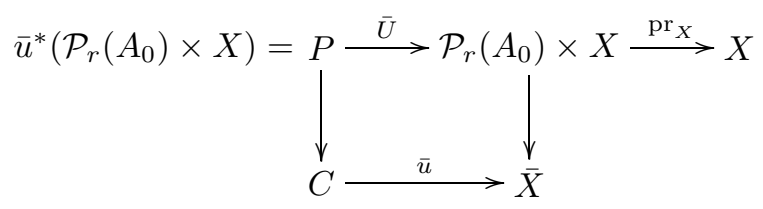

The composed map $U:=\operatorname{pr}_{X} \circ \bar{U}$ will be a $K$-equivariant, $A$-holomorphic map, for $A:=\bar{u}^{*} \mathcal{A}$ (see 5.2.3 for the definition of $\mathcal{A}$ ). As marked points in $P$, one may take any $\underline{p}$ lying over $\underline{x}$.

We have to prove that $F$ is injective. Consider $U:(P, A, p) \rightarrow X$ and $U^{\prime}$ : $\left(P, A^{\prime}, \underline{p}^{\prime}\right) \rightarrow X$ which induce the same morphism $\bar{u}: C \rightarrow \bar{X}$. It follows from definition (6.5) that necessarily $h\left(f_{A} A-A_{0}\right) \equiv h\left(f_{A^{\prime}} A^{\prime}-A_{0}\right) \bmod \mathbb{H}^{1}\left(C ; \mathbb{Z}^{r}\right)$, so that $h\left(f_{A} A-f_{A^{\prime}} A^{\prime}\right) \equiv 0 \bmod \mathbb{H}^{1}\left(C ; \mathbb{Z}^{r}\right)$. Remark 5.1.4 implies that $f_{A} A$ and 
$f_{A^{\prime}} A^{\prime}$ are in the same $\mathscr{G}_{0}^{c}(P)$-orbit and consequently $A$ and $A^{\prime}$ are also in the same $\mathscr{G}_{0}^{c}(P)$-orbit. Since $\bar{u}$ is gauge invariant, we may assume that $A=A^{\prime}$ and even that $A-A_{0}$ is a harmonic form.

The problem is reduced to the following: two maps $U:(P, A, p) \rightarrow X$ and $U^{\prime}:\left(P, A, \underline{p}^{\prime}\right) \rightarrow X$ which define the same $(\bar{u}, \bar{x})$ must be equal. Formula (6.6) says that

$$
\left[\left[A-A_{0}, p_{\zeta}\right], U\left(p_{\zeta}\right),[\underline{p}]\right]=\left[\left[A-A_{0}, p_{\zeta}\right], U^{\prime}\left(p_{\zeta}\right),\left[\underline{p}^{\prime}\right]\right] \quad \forall p_{\zeta} \in P .
$$

A moment's thought shows that this imply $U=U^{\prime}$ and $[\underline{p}]=\left[p^{\prime}\right]$.

Remark 6.3. An advantage of working with $M_{C, k}(\bar{X} ; \bar{B})$ is that it has a natural quasi-projective scheme structure. This was proved in lemma 3.1 , where is also described the construction of its compactification $\bar{M}_{C, k}(\bar{X} ; \bar{B})$ in terms of stable maps. Certainly, working within this algebraic frame has its own disadvantages: the space of stable maps may be badly behaved or it may have wrong dimension; an instance of a very unpleasant situation is when $M_{C, k}(\bar{X} ; \bar{B}) \subset \bar{M}_{C, k}(\bar{X} ; \bar{B})$ is not dense.

When $\bar{M}_{C, k}(\bar{X} ; \bar{B})$ has larger dimension than the expected one, it seems possible to introduce a virtual class on $\bar{M}_{C, k}(\bar{X} ; \bar{B})$ using obstruction theory relative to $\pi: \bar{X} \rightarrow\left(\mathrm{Pic}^{0} \mathrm{C}\right)^{r} \times C$. This should correspond to the limit of the fundamental cycles of the moduli spaces of pseudo-holomorphic curves to $(\bar{X}, \bar{J}):=$ $\mathcal{P}_{r}\left(A_{0}\right) \times_{K}(X, J)$, with $J$ a $K$-invariant, generic almost complex structure on $X$. Unfortunately, for the moment, I can not make this statement more precise.

There is a natural $G$-action on $\bar{X}$ :

$$
g \times[[A, p], x]:=[[A, p], g x] \quad \text { for } \quad[[A, p], x] \in \bar{X}=\mathcal{P}_{r}^{c}\left(A_{0}\right) \times_{G} X
$$

which is well-defined precisely because $G$ is commutative. The $G$-action can be linearized in the line bundle

$$
L:=\mathcal{P}_{r}^{c}\left(A_{0}\right) \times{ }_{G} \mathcal{O}_{X}(1) \longrightarrow \bar{X},
$$

which is $\pi$-ample. For $\ell \rightarrow\left(P i c^{0} C\right)^{r} \times C$ sufficiently ample line bundle,

$$
\bar{L}:=\pi^{*} \ell \otimes L \longrightarrow \bar{X}
$$

is ample and the $G$-action linearizes again. In is rather clear that the set of $G$ semi-stable points of $\bar{X}$ for this action is $\bar{X}^{\text {ss }}=\mathcal{P}_{r}^{c}\left(A_{0}\right) \times_{G} X^{\text {ss }}$. In particular, if $G$ acts freely on $X^{\text {ss }}$, it will does the same on $\bar{X}^{\text {ss }}$. The invariant quotient is, in any case,

$$
\bar{X} / / G=\left(P i c^{0} C\right)^{r} \times C \times \hat{X} \quad \text { where } \quad \hat{X}:=X / / G .
$$

The next lemma is useful to "visualize" better $M_{C, k}(\bar{X} ; \bar{B})$.

Lemma 6.4. Assume that $G$ acts freely on $X^{\mathrm{ss}}$ and let $\phi: X^{\mathrm{ss}} \rightarrow \hat{X}$ be the quotient map. Consider $\bar{u} \in M_{C, k}(\bar{X} ; \bar{B})$ with Image $(\bar{u}) \subset \mathcal{P}_{r}^{c}\left(A_{0}\right)_{\tau} \times_{G} X^{\mathrm{ss}}$. Then $(\phi \circ \bar{u})^{*} X^{\mathrm{ss}} \rightarrow C$ represents the point $\tau \in\left(P i c^{0} C\right)^{r}$. 
Proof. Notice that in the diagram

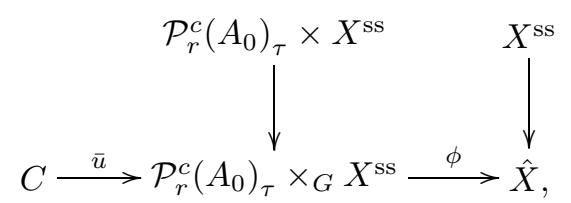

$\mathcal{P}_{r}^{c}\left(A_{0}\right)_{\tau} \times X^{\mathrm{ss}}=\phi^{*} X^{\mathrm{ss}}$. Indeed, for $\left([p, x], x^{\prime}\right) \in \phi^{*} X^{\mathrm{ss}}$ there is a unique $p^{\prime}$ such that $\left[p^{\prime}, x^{\prime}\right]=[p, x]$, so we may identify $\left([p, x], x^{\prime}\right)=\left(p^{\prime}, x^{\prime}\right)$. Consequently, $(\phi \circ \bar{u})^{*} X^{\mathrm{ss}}=\bar{u}^{*}\left(\mathcal{P}_{r}^{c}\left(A_{0}\right)_{\tau} \times X^{\mathrm{ss}}\right)$ and we obtain a $G$-equivariant map $(\phi \circ \bar{u})^{*} X^{\mathrm{ss}} \rightarrow \mathcal{P}_{r}^{c}\left(A_{0}\right)_{\tau}$ which covers the identity of $C$. This one must be an isomorphism.

We should recall that for obtaining the moduli space $S_{C, k}(X ; B)$ we still need to divide out the remaining $G$-action on $\bar{M}_{C, k}(\bar{X} ; \bar{B})$. For comparing the two points of view, the real-analytic and the algebraic one, we shall use the results obtained in section 3: the moment map corresponding to the $G$-action on $\bar{X}$ is the function $\bar{m}$ defined by

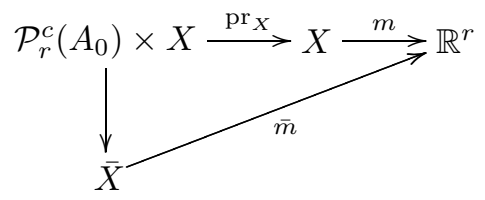

It follows from remark 3.6 that the limit moment map on $\bar{M}_{C, k}(\bar{X} ; \bar{B})$ is

$$
\bar{u} \longmapsto \int_{C} \bar{m} \circ \bar{u} \in \mathbb{R}^{r}
$$

and the invariant quotient is constructed dividing the zero level set by $K=\left(S^{1}\right)^{r}$.

On the other hand, the zero level $\left\{* F_{A}+m \circ U=0\right\}$ (modulo gauge) appearing in the definition 6.1 can be written also as

$$
U \longmapsto \int_{C} m \circ U=-\int_{C} F_{A}=\underline{\delta} \in \mathbb{R}^{r},
$$

where $\underline{\delta}$ represents the multi-degree of the $\left(S^{1}\right)^{r}$-bundle $P$, which is a topological invariant. We deduce that $S_{C, k}(X ; B)$ defined by $(6.2)$ and $\bar{M}_{C, k}(\bar{X} ; \bar{B}) / / G$ should be birational because both of them are Marsden-Weinstein quotients of $\bar{M}_{C, k}(\bar{X} ; \bar{B})$. However, unless $\bar{M}_{C, k}(\bar{X} ; \bar{B})$ is irreducible, this issue can be quite tricky.

In the particular case when $\underline{\delta}=0$, that is we consider bundles with multi-degree $(0, \ldots, 0)$, the real-analytic and the algebro-geometric constructions coincide. 


\section{Hamiltonian invariants}

Before stating the main result of this section, we need some notations. We define the evaluation maps

$$
\begin{gathered}
\widetilde{\mathrm{EV}}_{k}: \mathfrak{X}_{B} \times\left(P^{c}\right)_{o}^{k} / \mathscr{G}_{0}^{c}(P) \longrightarrow X^{k} \\
{\left[(U, A),\left(p_{1}, \ldots, p_{k}\right)\right] \longmapsto\left(U\left(p_{1}\right), \ldots, U\left(p_{k}\right)\right)}
\end{gathered}
$$

and

$$
\begin{gathered}
\operatorname{Ev}_{k}: \mathfrak{X}_{B}^{\mathrm{s}} \times\left(P^{c}\right)_{o}^{k} / \mathscr{G}^{c}(P) \longrightarrow X^{k} \\
{\left[(U, A),\left(p_{1}, \ldots, p_{k}\right)\right] \longmapsto\left(U\left(p_{1}\right), \ldots, U\left(p_{k}\right)\right)}
\end{gathered}
$$

and

$$
\begin{gathered}
\overline{\mathrm{EV}}_{k}: \mathfrak{X}_{B} \times\left(P^{c}\right)_{o}^{k} / \mathscr{G}_{0}^{c}(P) \longrightarrow\left(\mathcal{P}_{r}^{c}\left(A_{0}\right) \times X\right)^{k}, \\
{[(U, A), \underline{p}] \longmapsto\left(\left[h\left(A-A_{0}\right), R_{f_{A}} \underline{p}\right], U(\underline{p})\right) .}
\end{gathered}
$$

Computation (6.7) shows that the last evaluation is well-defined. All of them are $G^{k}$-equivariant and the last map induces on $M_{C, k}(\bar{X} ; \bar{B})$ the usual evaluation

$$
e v_{k}: M_{C, k}(\bar{X} ; \bar{B})=\mathfrak{X}_{B} \times\left(P^{c}\right)_{o}^{k} / \mathscr{G}_{0}^{c}(P) \times G^{k} \longrightarrow \bar{X}^{k} .
$$

The key for understanding the relationship between the analytic point of view and the algebraic one developed in the present paper is the diagram

$$
\begin{aligned}
& \mathfrak{X}_{B}^{\mathrm{s}} \times\left(P^{c}\right)_{o}^{k} / \mathscr{G}_{0}^{c}(P) \\
& \text { quot out the free } \\
& \downarrow G \text {-action (for } k \geq 1 \text { ) } \\
& \mathfrak{X}_{B}^{\mathrm{s}} \times\left(P^{c}\right)_{o}^{k} / \mathscr{G}^{c}(P) \\
& \downarrow \text { quot out the (not neces- } \\
& \subset \quad \mathfrak{X}_{B} \times\left(P^{c}\right)_{o}^{k} / \mathscr{G}_{0}^{c}(P) \\
& \downarrow \begin{array}{l}
\text { quot out the free } \\
G^{k} \text {-action }
\end{array} \\
& \mathfrak{X}_{B} \times\left(P^{c}\right)_{o}^{k} / \mathscr{G}_{0}^{c}(P) \times G^{k} \\
& S_{C, k}(X ; B) \leftrightarrow M_{C, k}(\bar{X} ; \bar{B}) / / G \quad \leftrightarrow \quad M_{C, k}(\bar{X} ; \bar{B}) .
\end{aligned}
$$

Since $G / K\left(=\mathbb{R}_{>0}^{r}\right)$ is contractible, $K$ and $G$-equivariant cohomologies of $X$ coincide; we shall prefer $G$-equivariant classes. Recall that $2 D$ denotes the expected dimension of $S_{C, k}(X ; B)$ and its formula is given by $(6.3)$.

Definition 7.1. The Hamiltonian invariant introduced in $[6,14]$ is defined in the following way: consider an equivariant cohomology class $\alpha \in H_{G}^{*}(X)^{\otimes k}$ with $\operatorname{deg} \alpha=2 D$. Under the assumption that $G^{k}$ acts freely on $\mathfrak{X}_{B}^{\mathrm{s}} \times\left(\mathcal{P}^{c}\right)_{o}^{k} / \mathscr{G}^{c}(P)$, the pull-back defines a cohomology class on $S_{C, k}(X ; B)$ denoted the same. The invariant is

$$
\Phi_{X, B}^{C, k}(\alpha):=\int_{S_{C, k}(X ; B)}\left(\mathrm{EV}_{k}\right)^{*} \alpha
$$


I have to say that $\Phi$ is defined this way only when $S_{C, k}(X ; B)$ has the correct dimension. For this reason the authors in $[6,14]$ work with perturbations of $S_{C, k}(X ; B)$. In algebraic context, one should integrate over a $\pi$-relative virtual cycle, as mentioned in remark 6.3.

It is conjectured in [6] that for special choices of $\alpha$ and $B$ the invariant $\Phi$ should coincide with a Gromov-Witten invariant of $\hat{X}=X / / G$. More precisely,

Conjecture. Take $\alpha \in H_{G}^{*}(X)^{\otimes k}$ and $\hat{B} \in H_{2}(\hat{X} ; \mathbb{Z})$, with $\hat{X}:=X / / G$ and let $\hat{\alpha} \in H^{*}\left(\hat{X}^{k}\right)$ and $B \in H_{2}^{G}(X ; \mathbb{Z})$ be respectively the classes defined by

$$
\hat{X} \stackrel{\sim}{\longleftarrow} E \times_{G} X^{\mathrm{ss}} \longrightarrow E \times_{G} X .
$$

Then $\Phi_{C, k}^{X, B}(\alpha)=G W_{C, k}^{\hat{X}, \hat{B}}(\hat{\alpha})$.

Our goal is to prove this conjecture under the same transversality assumptions as in theorem 4.2, when the invariant homology class $B \in H_{2}^{G}(X ; \mathbb{Z})$ is induced from $X^{\mathrm{ss}}$; the reason for this restriction was discussed in the end of the last section. So we will deal with topologically trivial $\left(\mathbb{C}^{*}\right)^{r}$-bundles over a smooth curve $C$. For $\mathcal{P}_{r}^{c} \rightarrow\left(P i c^{0} C\right)^{r} \times C$ the universal $\left(\mathbb{C}^{*}\right)^{r}$-bundle (trivialized at a point $\zeta_{0} \in C$ ), we define $\bar{X}:=\mathcal{P}_{r}^{c} \times\left(\mathbb{C}^{*}\right)^{r} X$.

Theorem 7.2. Let the torus $G \cong\left(\mathbb{C}^{*}\right)^{r}$ act on the irreducible projective variety $X$, and consider a linearization of this action in the very ample line bundle $\mathcal{O}_{X}(1) \rightarrow$ $X$. Denote $B \in H_{2}(X ; \mathbb{Z})$ a class which can be represented by a morphism $C \rightarrow$ $X^{\mathrm{ss}}$, where $C$ is a smooth projective curve with $\operatorname{Aut}(C)=\left\{\operatorname{id}_{C}\right\}$, and let $\hat{B} \in$ $H_{2}(\hat{X} ; \mathbb{Z})$ be the class induced by the projection $\phi: X^{\mathrm{ss}} \rightarrow X^{\mathrm{ss}} / G=\hat{X}$. Suppose that

(a1) $G$ acts freely on the $G$-semi-stable locus of $X$, so that $X^{\mathrm{ss}} \rightarrow \hat{X}$ is a principal $G$-bundle and denote $\bar{B} \in H_{2}(\bar{X} ; \mathbb{Z})$ the class induced as in lemma 6.1 ;

(a2) $\bar{M}_{C, k}(\bar{X} ; \bar{B})$ is generically smooth and has the expected dimension;

(a3) every irreducible component of $\bar{M}_{C, k}(\bar{X} ; \bar{B})$ contains a point represented by a morphism $C \rightarrow \bar{X}^{\mathrm{ss}}=\mathcal{P}_{r}^{c} \times\left(\mathbb{C}^{*}\right)^{r} X^{\mathrm{ss}}$;

(a4) $M_{C, k}(\hat{X} ; \hat{B}) \subset \bar{M}_{C, k}(\hat{X} ; \hat{B})$ is dense.

Then for any $\alpha \in H_{G}^{*}(X)^{\otimes k}$,

$$
G W_{\hat{X}, \hat{B}}^{C, k}(\hat{\alpha})=\Phi_{X, B}^{C, k}(\alpha),
$$

where $\hat{\alpha} \in H^{*}(\hat{X})^{\otimes k}$ is the class induced by $\alpha$.

Proof. I start by explaining the guiding idea: we have learned in section 4 that we should transfer the integrals on $\bar{M}_{C, k}(\hat{X} ; \hat{B})$ used for defining Gromov-Witten invariants of $\hat{X}$ to integrals on $\bar{M}_{C, k}(\bar{X} ; \bar{B}) / / G$ because $\bar{X} / / G=\left(\operatorname{Pic}^{0} C\right)^{r} \times C \times \hat{X}$. All the evaluation maps involved for making these computations live on the righthand-side of (7.5). On the other hand, the invariant $\Phi$ is defined using equivariant 
cohomology classes which are pulled-back to $S_{C, k}(X ; B)$ by maps which live on the left-hand-side of (7.5). So, loosely speaking, what we have to do is to pass from the left to the right in (7.5).

For making this passage, we need to understand the relationship between the various group actions and evaluation maps which appear in the context. On $\mathfrak{X}_{B} \times$ $\left(P^{c}\right)_{o}^{k} / \mathscr{G}_{0}^{c}(P)$ there are two commuting actions. First, $G \subset \mathscr{G}^{c}(P)$ acts by constant complex gauges

$$
g \times[(U, A), \underline{p}]=\left[(g U, A), R_{g} \underline{p}\right] .
$$

The map $\widetilde{\mathrm{EV}}_{k}$ is $G$-invariant for this action, while $\overline{\mathrm{EV}}_{k}$ is $G$-equivariant for the diagonal right action of $G$ on the $\mathcal{P}_{r}^{c}$-factor of $\left(\mathcal{P}_{r}^{c} \times X\right)^{k}$.

Secondly, $G^{k}$ acts on $\mathfrak{X}_{B} \times\left(P^{c}\right)_{o}^{k} / \mathscr{G}_{0}^{c}(P)$ with quotient $M_{C, k}(\bar{X} ; \bar{B})$ :

$$
\left(g_{1}, \ldots, g_{k}\right) \times\left[(U, A),\left(p_{1}, \ldots, p_{k}\right)\right]=\left[(U, A),\left(R_{g_{1}} p_{1}, \ldots, R_{g_{k}} p_{k}\right)\right] .
$$

The evaluation map $\overline{\mathrm{EV}}_{k}$ is $G^{k}$-equivariant for the $G$-action on $\mathcal{P}_{r}^{c} \times X$ on both terms.

Convention. In what follows, the symbol " " will denote homotopy equivalence and the letter " $y$ " obvious inclusions. For understanding better the forthcoming calculations, we should keep in mind that for integration purposes homotopy equivalent spaces are equal.

$$
\begin{aligned}
S_{C, k}(X ; B) & \sim E^{k} \times_{G^{k}}\left(\mathfrak{X}_{B}^{\mathrm{s}} \times\left(P^{c}\right)_{o}^{k} / \mathscr{G}^{c}(P)\right) \\
& \sim E^{k} \times_{G^{k}}\left(E \times_{G}\left(\mathfrak{X}_{B}^{\mathrm{s}} \times\left(P^{c}\right)_{o}^{k} / \mathscr{G}_{0}^{c}(P)\right)\right) \\
& =E \times_{G}\left(E^{k} \times_{G^{k}}\left(\mathfrak{X}_{B}^{\mathrm{s}} \times\left(P^{c}\right)_{o}^{k} / \mathscr{G}_{0}^{c}(P)\right)\right) .
\end{aligned}
$$

The map

$$
\overline{\mathrm{EV}}_{k}: \mathfrak{X}_{B} \times\left(P^{c}\right)_{o}^{k} / \mathscr{G}_{0}^{c}(P) \longrightarrow\left(\mathcal{P}_{r}^{c} \times X\right)^{k}
$$

being $G^{k}$-equivariant and $G$-invariant, induces

$$
\begin{aligned}
& E \times_{G}\left(E^{k} \times_{G^{k}}\left(\mathfrak{X}_{B} \times\left(P^{c}\right)_{o}^{k} / \mathscr{G}_{0}^{c}(P)\right)\right) \stackrel{\sim}{\longrightarrow} E \times_{G} M_{C, k}(\bar{X} ; \bar{B}) \\
& E \times_{G}\left(E^{k} \times_{G^{k}}\left(\mathcal{P}_{r}^{c} \times X\right)^{k}\right) \longrightarrow E \times_{G} \bar{X}^{k} .
\end{aligned}
$$

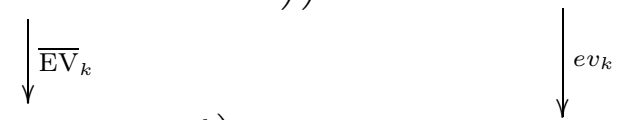

On the other hand,

$$
\begin{aligned}
& E \times_{G}\left(E^{k} \times_{G^{k}}\left(\mathcal{P}_{r}^{c} \times X\right)^{k}\right) \longrightarrow \times_{G} \bar{X}^{k} \\
& E \times_{G}\left(E^{k} \times_{G^{k}} X^{k}\right)=\mathrm{BG} \times\left(E^{k} \times_{G^{k}} X^{k}\right) .
\end{aligned}
$$


The reason for the last equality is that $G$ acts on $\mathcal{P}_{r}^{c}$ only and therefore the $G$-action on $E^{k} \times_{G^{k}} X^{k}$ is trivial. The class $\alpha$ we start with lives in $H_{G}^{*}(X)^{\otimes k}$; pulling it back using $\epsilon$, we get the class $\bar{\alpha} \in H_{G}^{*}\left(\bar{X}^{k}\right)$. Relation (7.7) implies that $\mathrm{EV}_{k}=\epsilon \circ \overline{\mathrm{EV}}_{k}$ and we deduce from diagrams $(7.8)$ and (7.9) that $\mathrm{EV}_{k}^{*} \alpha=e v_{k}^{*} \bar{\alpha} \in H_{G}^{*}\left(M_{C, k}(\bar{X} ; \bar{B})\right)$.

The invariant $\Phi$ can therefore be defined as

$$
\Phi(\alpha)=\int_{\bar{M}_{C, k}(\bar{X} ; \bar{B}) / / G} j_{M^{\mathrm{ss}}}^{*} e v_{k}^{*} \bar{\alpha},
$$

where the relevant maps fit in the diagram

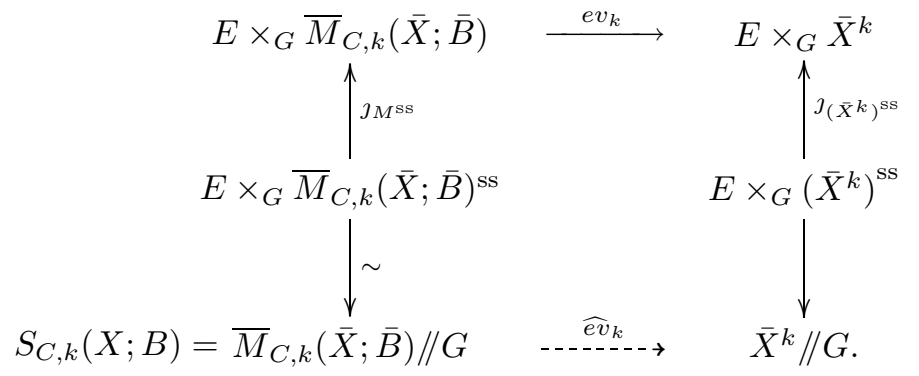

From the diagram

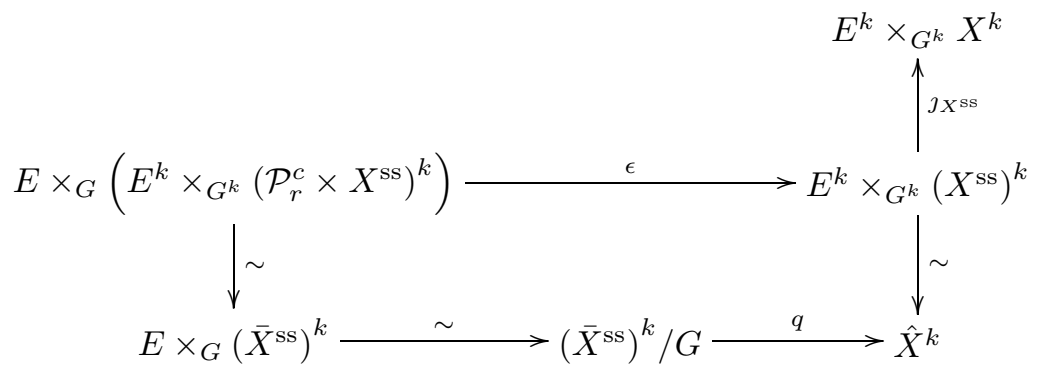

we see that $\jmath_{\bar{X}^{\mathrm{ss}}}^{*} \bar{\alpha}=\jmath_{\bar{X}^{\mathrm{ss}}}^{*} \epsilon^{*} \jmath_{X^{\mathrm{ss}}}^{*} \alpha=q^{*} \hat{\alpha}$, where $\hat{\alpha}$ is by definition the cohomology class on $\hat{X}^{k}$ determined by $\alpha$.

The assumption (a3) says that the subset $M_{C, k}(\bar{X} ; \bar{B})^{o} \subset \bar{M}_{C, k}(\bar{X} ; \bar{B})^{\mathrm{ss}}$ of morphisms with image contained in $\bar{X}^{\text {ss }}$ is dense. We deduce, going the other way round in (7.11), that

$$
\Phi(\alpha)=\int_{\bar{M}_{C, k}(\bar{X} ; \bar{B}) / / G}\left(\widehat{e v}_{k}\right)^{*} q^{*} \hat{\alpha} .
$$

At this point we have finally moved from the left-hand-side to the right-hand-side of (7.5) as we wished. Notice that since the maps involved in the computations are rational, the pull-backs are defined as in 4.1 .

The composition of morphisms $\bar{u}: C \rightarrow \bar{X}$ representing the class $\bar{B}$ with the projection $\bar{X} \rightarrow\left(\operatorname{Pic}^{0} C\right)^{r} \times C$ is of the form $\{\tau\} \times \mathrm{id}_{C}$ because $\bar{B}$ induces the class 
$0 \oplus[C]$. Consequently, a map $\bar{u}: C \rightarrow \bar{X}^{\text {ss }}$ defines a map $C \rightarrow C \times \hat{X}$ which is the identity on the first component.

Lemma 7.3. The map

$$
T: \bar{M}_{C, k}(\bar{X} ; \bar{B}) / / G \rightarrow \bar{M}_{C, k}(C \times \hat{X} ;[C]+\hat{B})
$$

is birational.

Proof. Assumption (a4) implies that $T$ is dominant if every morphism $\hat{u}: C \rightarrow \hat{X}$ representing $\hat{B}$ is in its image. From the diagram

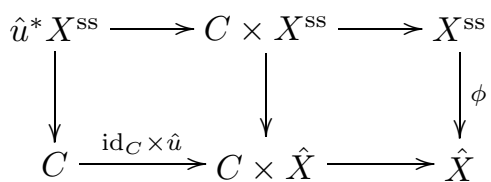

we deduce that the pull-back $\hat{u}^{*} X^{\mathrm{ss}} \rightarrow C$ is a topologically trivial, holomorphic principal bundle (this follows from the assumption that $\hat{B}$ is induced by a class $\left.B \in H_{2}\left(X^{\mathrm{ss}} ; \mathbb{Z}\right)\right)$; it is therefore isomorphic to $\mathcal{P}_{\tau}:=\left.\mathcal{P}_{r}^{c}\right|_{\{\tau\} \times C}$ for a certain $\tau \in$ $\left(P i c^{0} C\right)^{r}$. This data induces the map $\bar{u}: C \rightarrow \mathcal{P}_{\tau} \times_{G} X^{\mathrm{ss}} \subset \mathcal{P}_{r}^{c} \times_{G} X=\bar{X}$ which represents the class $\bar{B}$ and also $T(\bar{u})=\hat{u}$.

Now let us prove that $T$ is generically injective. Using assumption (a3), we may restrict ourselves to the (dense) open subset $M_{C, k}(\bar{X} ; \bar{B})^{o}$ representing morphisms whose image is contained in $\bar{X}^{\text {ss }}$. Let us assume that

$$
\begin{aligned}
& C \stackrel{\bar{u}}{\longrightarrow} \mathcal{P}_{\tau} \times_{G} X_{\searrow^{\mathrm{ss}}} \\
& C \times \hat{X} \\
& C \stackrel{\bar{u}^{\prime}}{\longrightarrow} \mathcal{P}_{\tau^{\prime}} \times_{G} X^{\mathrm{ss}}{ }^{\nearrow_{\phi}}
\end{aligned}
$$

are such that $\phi \circ \bar{u}=\phi \circ \bar{u}^{\prime}$. Then $(\phi \circ \bar{u})^{*}\left(X^{\mathrm{ss}} \rightarrow \hat{X}\right)=\left(\phi \circ \bar{u}^{\prime}\right)^{*}\left(X^{\mathrm{ss}} \rightarrow \hat{X}\right)$ and lemma 6.4 implies that $\tau=\tau^{\prime}$. Then $C \stackrel{\bar{u}, \bar{u}^{\prime}}{\longrightarrow} \mathcal{P}_{\tau} \times_{G} X^{\text {ss }}$ induce the same map to $\hat{X}$ and consequently for any $\zeta \in C, \bar{u}^{\prime}(\zeta)=g(\zeta) u(\zeta)$ for a unique $g(\zeta) \in G$. The morphism $C \rightarrow G$ must be constant, so $\bar{u}^{\prime}=g \bar{u}$ and they define the same point in $\bar{M}_{C, k}(\bar{X} ; \bar{B}) / / G$.

Since $C$ has trivial automorphism group,

$$
\bar{M}_{C, k}(C \times \hat{X} ;[C]+\hat{B}) \leftrightarrow{ }^{1: 1} \rightarrow-\bar{M}_{C, k}(\hat{X} ; \hat{B}) .
$$

Finally, from the diagram

$$
\begin{gathered}
\bar{M}_{C, k}(\bar{X} ; \bar{B}) / / G \stackrel{\widehat{e v}_{k}}{\cdots} \bar{X}^{k} / / G \stackrel{q}{\cdots} \hat{X}^{k} \\
1: 1 \\
\downarrow \\
\bar{M}_{C, k}(C \times \hat{X} ;[C]+\hat{B}) \stackrel{e v_{k}^{\hat{X}}}{\longrightarrow}(C \times \hat{X})^{k} \stackrel{\operatorname{pr}_{\hat{X}}}{\longrightarrow} \hat{X}^{k}
\end{gathered}
$$


we conclude that the invariant $\Phi$ does coincide with a Gromov-Witten invariant of $X$. Indeed,

$$
\begin{aligned}
\Phi_{X, B}^{C, k}(\alpha) & =\int_{\bar{M}_{C, k}(\bar{X} ; \bar{B}) / / G} \widehat{e v}_{k}^{*} q^{*} \hat{\alpha}=\int_{\bar{M}_{C, k}(C \times \hat{X} ;[C]+\hat{B})}\left(e v_{k}^{\hat{X}}\right)^{*} \operatorname{pr}_{\hat{X}}^{*} \hat{\alpha} \\
& =\int_{\bar{M}_{C, k}(\hat{X} ; \hat{B})}\left(e v_{k}^{\hat{X}}\right)^{*} \hat{\alpha} .=G W_{\hat{X}, \hat{B}}^{C, k}(\hat{\alpha}) .
\end{aligned}
$$

I would like to conclude with the remark

Why should the Hamiltonian invariants be interesting? I have mentioned in the introduction that the starting point of this study was the problem of comparing the GW-invariants of a quotient with the invariants of the variety we start with. Simple dimensional counting shows that -except in genus zero- the question is not well-posed in this form: the dimension of the space of morphisms from curves to a quotient variety is larger than the dimension of the space of morphisms into the starting variety. The difference between these dimensions is exactly the dimension of the moduli space of principal $G$-bundles over a curve; this can be explained noticing that, for morphisms $v: C \rightarrow \hat{X}$ which represent an a priori given homology class, the holomorphic type of the the pull-backs $v^{*} X^{\mathrm{ss}} \rightarrow C$ changes within a fixed topological type. In this way, the space of principal bundles over curves with fixed topological type naturally enters into the scene.

Equality (7.12) brings our attention to another aspect of the problem: GWinvariants of $\hat{X}$ can be computed (under suitable transversality conditions) in the following way

$$
G W_{\hat{X}, \hat{B}}^{C, k}(\hat{\alpha})=\int_{\bar{M}_{C, k}(\bar{X} ; \bar{B}) / / G}\left(q \circ \widehat{e v}_{k}\right)^{*} \hat{\alpha},
$$

for $q:\left(\bar{X}^{\mathrm{ss}}\right)^{k} / G \rightarrow \hat{X}^{k}$. The interesting part is that there is a natural projection

$$
\pi: \bar{M}_{C, k}(\bar{X} ; \bar{B}) / / G \rightarrow\left(P i c^{0} C\right)^{r},
$$

so that one can further write

$$
G W_{\hat{X}, \hat{B}}^{C, k}(\hat{\alpha})=\int_{\left(P i c^{0} C\right)^{r}} \pi_{*}\left(q \circ \widehat{e v}_{k}\right)^{*} \hat{\alpha}
$$

and now the integration takes place on the Picard torus of the curve. One may hope that enumerative invariants of suitably chosen $\hat{X}$ 's can be expressed in terms of interesting intersection numbers of the Picard variety.

\section{References}

[1] K. Behrend and B. Fantechi, The intrinsic normal cone, Invent. Math. 128 (1997), 45-88.

[2] K. Behrend and Yu. Manin, Stacks of stable maps and Gromov-Witten invariants, Duke Math. J. 85 (1996), 1-60. 
[3] J.-M. Bismut and D. S. Freed, The analysis of elliptic families, metrics and connections on determinant bundles, Commun. Math. Phys. 106 (1986), 159-176.

[4] J.-M. Bismut and D. S. Freed, The analysis of elliptic families, Dirac operators, Eta invariants and the holonomy theorem, Commun. Math. Phys. 107 (1986), 103-163.

[5] J.-M. Bismut, H. Gillet and Ch. Soulé, Analytic torsion and holomorphic determinant bundles, Commun. Math. Phys. 115 (1988), 49-78, 79-126, 301-351.

[6] K. Cieliebak, A. R. Gaio and D. Salamon, J-holomorphic curves, moment maps and invariants of Hamiltonian group actions, Internat. Math. Res. Notices 16 (2000), 831-882.

[7] A. Grothendieck, Techniques de construction et théorèmes d'existence en géométrie algébrique IV, les schemas de Hilbert, Séminaire Bourbaki 1960/1961, No. 221.

[8] W. Fulton, Intersection Theory, Springer-Verlag, Berlin-Heidelberg-New York-Tokyo, 1984.

[9] W. Fulton and R. Pandharipande, Notes on stable maps and quantum cohomology in: J. Kollár et al., Algebraic Geometry Santa Cruz 1995, AMS 1997, Part 2, 45-96.

[10] F. Kirwan, Cohomology of Quotients in Symplectic and Algebraic Geometry, Princeton University Press, 1984.

[11] J. Li and G. Tian, Virtual moduli cycles and Gromov-Witten invariants, J. Amer. Math. Soc. 11 (1998), 119-174.

[12] D. Luna, Slices étales, Bull. Soc. Math. France 33 (1973), 81-105.

[13] D. Mumford, J. Fogarty and F. Kirwan, Geometric Invariant Theory, Springer Verlag, Berlin-Heidelberg-New York-London, 1994.

[14] I. Mundet i Riera, Hamiltonian Gromov-Witten invariants, preprint AG/0002121.

[15] A. Ramanathan, Stable principal bundles on a compact Riemann surface, Math. Ann. 213 (1975), 129-152.

[16] G. Tian, On a set of polarized Kaehler metrics on algebraic manifolds, J. Differ. Geom. 32 (1990), 99-130.

[17] J. J. Thomsen, Irreducibility of $\bar{M}_{0, n}(G / P, \beta)$, Internat. J. Math. 9 (1998), 367-376.

M. Halic

Current address:

Universität Zürich

Institut für Mathematik

Winterthurerstrasse 190

CH-8057 Zürich

Switzerland

e-mail: halic@math.unizh.ch

On leave from:

Ruhr-Universität Bochum

Fakultät für Mathematik

D-44780 Bochum

Germany

(Received: May 25, 2000; revised version: December 5, 2000) 